\title{
TUDelft
}

Delft University of Technology

Faculty of Civil Engineering and Geosciences

Department of Geosciences and Engineering

\section{Geologically relevant framework for adaptive fractured reservoir modeling}

Thesis submitted to the Delft Institute of Applied Earth Sciences in partial fulfillment of the requirements for the degree

\section{MASTER OF APPLIED EARTH SCIENCES}

\author{
by
}

\section{Daan Robert Ris}

D.R.Ris@student.tudelft.nl

Delft, the Netherlands

Copyright (C) 2020 Daan Ris. All rights reserved.

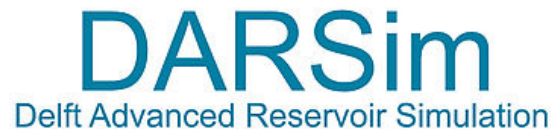





\author{
Author \\ Daan Ris \\ D.R.Ris@student.tudelft.nl
}

MSc Student, Petroleum Engineering \& Geosciences Department of Geosciences and Engineering Delft University of Technology

\section{Title}

Geologically relevant framework for adaptive fractured reservoir modeling

\section{Advisors}

Dr. Hadi Hajibeygi

H.Hajibeygi@tudelft.nl

S. Mousa HosseiniMehr

S.HosseiniMehr@student.tudelft.nl
Principal Advisor, Associate Professor

Department of Geoscience and Engineering

Delft University of Technology

Daily Supervisor, PhD Candidate

Department of Geosciences and Engineering Delft University of Technology

\section{Committee}

Bill Rossen

W.R.Rossen@tudelft.nl

Committee, Professor

Department of Geoscience and Engineering Delft University of Technology

Cornelis Vuik

C.Vuik@tudelft.nl

Committee, Professor

Department of Applied Mathematics

Delft University of Technology

Copyright (C) 2020 Daan Ris. All rights reserved. 



\begin{abstract}
It is a great challenge to accurately model the flow through subsurface reservoirs. The fact that they are located a few kilometers below the earth's surface means that it is very difficult to locate flow affecting phenomena. Fractures are considered to be such phenomena. They can either enhance or block flow which has a substantial effect on the flow through the reservoir. This research addresses the flow properties of fractures and the location of fractures. This research introduces analogue fracture maps to get a grasp on possible fracture locations and introduces a classification of fractures based on their orientation with respect to subsurface stress. Using this classification, fractures are assigned different permeability values, a flow affecting property.

Using this approach a fracture map is obtained that can be used to model flow through a reservoir. To model flow, the projection based Embedded Discrete Fracture Model (pEDFM) is implemented. This method uses independent grids for both the matrix and fracture and couples them using a transfer function.

Even when using the pEDFM method, the computational costs for running simulations through a field scale model will be too large. Therefor the concept of multiscale is introduced and developed further in this research. This research uses algebraically calculated basis functions to prolong the coarse scale solution to finescale. Besides this, flexible coarsening is introduced for a static Multilevel Multiscale (MMs) modeling approach and for Adaptive Dynamic Modeling (ADM). This allows the user to independently choose coarsening ratios for matrix and fractures.

This research shows that fractures can be represented accurately using only two vertices on a coarse grid. This can reduce computational costs. Besides the flexible coarsening, the research shows how the grid refinement scheme can be used in efficiently in multiphase simulations. Using a grid refinement scheme enables the user to capture a saturation front and to use a multiscale grid at the same time. The grid refinement scheme works most efficiently when the cells belonging to high permeable fractures are coupled to the cells belonging to the matrix.

The finescale solution This research finally shows the impact of geological uncertainty. The sensitivity analysis shows how geological uncertainty can have an influential impact on the problem that has to be solved. In the sensitivity analysis it is researched how a change in subsurface stress affects the finescale solutions.
\end{abstract}





\section{Acknowledgments}

In this section I would like to thank those who have made it possible to work on this research. First of all I want to thank Dr. Hadi Hajibeygi for the inspiration, motivation and confidence throughout my work. There was no meeting without an interesting insight or fruitful discussion. No matter how occupied, I always had the feeling I could address him for advice.

Next, I want to thank PhD candidate Moussa HosseiniMehr as working together closely has left a great impression. The many (virtual) meetings have been a joy to participate in and have taught me a lot. These meetings have made a positive impact on the research.

Also to the other members of my exam committee, Prof. dr. Bill Rossen and Prof, dr. Kees Vuik, I would like to show my gratitude. Besides my exam committee the Darsim Research Group deserves to be mentioned here as they have helped along the way in the form of interesting discussions, meetings and insights.

Lastly, I want to thank everyone who supported me throughout the project on a personal level. Without friends and family this research would not have been possible. A special mention should be given here to my parents and my girlfriend Juanita, who have never failed to motivate me to keep going.

Daan Ris

November 2020, Delft 



\section{Contents}

$\begin{array}{lr}\text { Acknowledgments } & 2\end{array}$

Lists

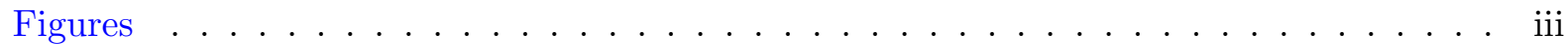

1 Introduction $\quad 3$

1.1 Fracture Characteristics . . . . . . . . . . . . . . . . . . 3

1.2 Modeling Fractures . . . . . . . . . . . . . . . . . . . . . . 4

1.3 Discrete Fracture Model $(\mathrm{DFM}) \ldots \ldots \ldots \ldots \ldots \ldots$

1.4 Multilevel Simulation Strategies . . . . . . . . . . . . . . . . . . 5

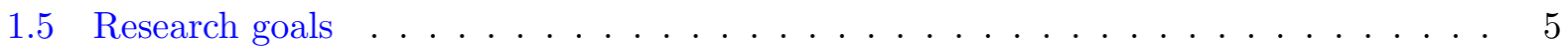

2 Obtaining Realistic Fracture Maps $\quad 7$

2.1 Fracture maps . . . . . . . . . . . . . . . . . . . 7

2.2 Fracture Classification . . . . . . . . . . . . . . . . 8

2.2 .1 Coulomb Friction Criterion $\ldots \ldots \ldots \ldots \ldots$

3 Solving strategy for EDFM method $\quad 11$

3.1 EDFM Concept . . . . . . . . . . . . . . . . . . . . . 11

3.2 Governing Equations . . . . . . . . . . . . . . . . . . . . 12

3.3 Connectivity Index . . . . . . . . . . . . . . . . . . . . . . 13

3.4 Intersecting Fractures $\ldots \ldots \ldots \ldots \ldots \ldots \ldots \ldots$

3.5 pEDFM Implementation . . . . . . . . . . . . . . . . . . . . 15

3.6 Fully Implicit Discretization . . . . . . . . . . . . . . . . . . . . . 16

4 Multiscale Methods for Flow and Pressure $\quad 19$

4.1 Multilevel Multiscale $(\mathrm{MMs}) \ldots \ldots \ldots \ldots$. . . . . . . . . . . . . 19

4.2 Implementation Strategy . . . . . . . . . . . . . . . . . . . . . . 19

4.3 Basis functions for Multiscale . . . . . . . . . . . . . . . 20

4.3 .1 Coarse Scale Grids . . . . . . . . . . . . . . . . . . . . 20

4.3 .2 Flexible Coarsening for Fractures . . . . . . . . . . . . . . . . 22

4.3.3 Computation of Basis Functions _ . . . . . . . . . . . . . . 22

4.3 .4 Vertices on Fractures . . . . . . . . . . . . . . . . . . . 23

4.4 Reducing Computational Cost by Using Multilevel . . . . . . . . . . . . . . . . . 25

4.5 Algebraic Dynamic Multiscale $(\mathrm{ADM}) \ldots \ldots \ldots \ldots \ldots \ldots$

5 Static Multilevel Multiscale Results $\quad 29$

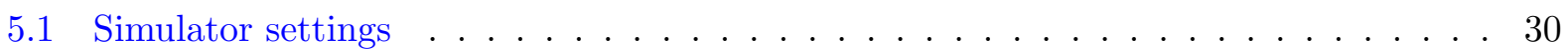

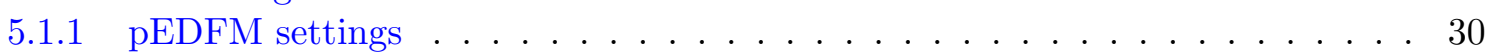

5.2 Cross Test Case . . . . . . . . . . . . . . . . . . . . . 31

5.2 .1 Fully Permeable Cross . . . . . . . . . . . . . . . . . . . 31

5.2 .2 Fully Impermeable Cross $\ldots \ldots \ldots$. . . . . . . . . . . . . . 33 
5.2.3 Vertical Fracture Permeable, Horizontal Fracture Impermeable . . . . . . . 35

5.2.4 Horizontal Fracture Permeable, Vertical Fracture Impermeable . . . . . . . . 37

5.2 .5 Error table . . . . . . . . . . . . . . . . . . . 38

5.3 Benchmark 1 Test Case . . . . . . . . . . . . . . . . . . . . . 39

5.3.1 Horizontal Flow, Benchmark Settings . . . . . . . . . . . . . . . 39

5.3 .2 Vertical Flow, Benchmark Settings . . . . . . . . . . . . . . . . . . . 41

5.3 .3 Permeable Fractures, Horizontal Flow . . . . . . . . . . . . . . . . . . 43

5.3 .4 Impermeable Fractures, Horizontal Flow . . . . . . . . . . . . . . . . . . 45

5.3 .5 Error Table . . . . . . . . . . . . . . . . . . . 47

5.4 Benchmark 2 Test Case . . . . . . . . . . . . . . . . . . . . . . 48

5.5 Influence of pEDFM on Results . . . . . . . . . . . . . . . . . . 50

5.6 Benchmark 1, No Basis Function Adjustments . . . . . . . . . . . . . . 50

6 Algebraic Dynamic Modelling Results $\quad \mathbf{5 5}$

6.1 Simulator Settings . . . . . . . . . . . . . . . . . . 55

6.2 Flexible Coarsening Ratios for Fractures . . . . . . . . . . . . . . . . . 57

6.3 Coupling of Fractures . . . . . . . . . . . . . . . . . . 58

6.4 Field Scale Sensitivity Analysis . . . . . . . . . . . . . . . . . . . 6 61 . . . . . . . .

6.4 .1 Field Scale Applicability . . . . . . . . . . . . . . . . . . 61

6.4 .2 Sensitivity to Geological Uncertainty . . . . . . . . . . . . . . . . . 62

7 Conclusion and Discussion $\quad 65$

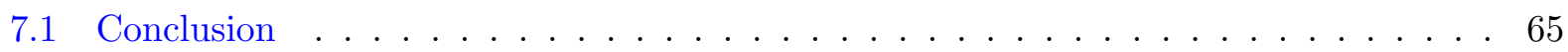

7.2 Discussion . . . . . . . . . . . . . . . . . . . 66

\section{Appendices}

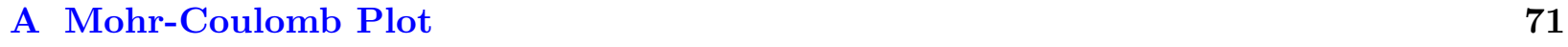

$\begin{array}{ll}\text { B Principle Stress Settings } & 73\end{array}$

$\begin{array}{ll}\text { C Pressure Plot ADM Test Case } & \mathbf{7 5}\end{array}$

C.1 Flexible Coarsening Ratios for Fractures . . . . . . . . . . . . . . . . . . . 75

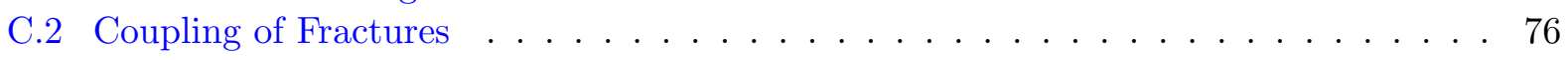




\section{Figures}

1.1 Fractures in geological formations at different scales. (a) Fractures visible from a drone image in Apodi, Brazil (photo taken by Kevin Bisdom [1]). (b) Fractures visible in an outcrop in the Western High Atlas, Morocco (photo taken by the author).

1.2 The concept of upscaling fractures visualized. In a fractured matrix a grid is imposed and where the fractures cross these grids the properties belonging to these grids are modified to adjust for the presence of fractures $[2] \ldots \ldots \ldots \ldots$

2.1 Mohr's circle for stress and failure envelopes. The coefficient of static friction on pre-existing planes with a value of $\mu=0.6$ and the principle stresses, $\sigma_{1}$ and $\sigma_{3}$ are plotted to define the range of $\beta_{1}$ to $\beta_{2}$ in which fractures will be critically stressed.

2.2 A fracture shown that is optimally orientated for frictional sliding in a 2-dimensional field.

2.3 (a) The location of the fractures as interpreted by Kevin Bisdom [3] (b) The fractures with an assigned permeability . . . . . . . . . . . . .

3.1 The concept of EDFM visualized. In figure a) the matrix and 3 fractures can be seen. This matrix is discretized with a uniform, structured grid which can be seen in b.1). The fractures are also discretized but separately in figure b.2). After coupling the matrix and fractures through a transfer function they are brought together in c).

3.2 Examples of calculating the area fraction of the corresponding fracture element $k$ in matrix cell $(i, j) \ldots \ldots \ldots \ldots \ldots \ldots \ldots \ldots \ldots \ldots$

3.3 An intersection of two fractures. The blue dots represent the cell centers of the fractures. . . . . . . . . . . . . . . . . . . .

3.4 Illustration of the pEDFM method. The matrix cells of this $2 \mathrm{D}$ structured grid containing a single $1 \mathrm{D}$ fracture that are overlapped by the fracture are shown in blue. These are directly connected, as defined in classic EDFM. The yellow cells in the zoomed in view are the cells that take part in the additional non-neighboring connections between fracture and matrix grid cells.

4.1 Here a coarse grid is projected on a fine scale grid. Using the formula 4.3.1: $\left(N_{c}-\right.$ $1) \cdot \gamma^{l}+1=(4-1) \cdot 5^{1}+1=16$. So 16 grids in both $\mathrm{x}$ and $\mathrm{y}$ direction are used. . . 21

4.2 Here a coarse grid is projected on a fine scale grid. Using the formula 4.3.2: $N_{c} \cdot \gamma^{l}=$ $(3) \cdot 5^{1}+1=15$. So 15 grids in both $\mathrm{x}$ and $\mathrm{y}$ direction are used. . . . . . . . 22

4.3 A 1-D representation of a coarse grid. This is implemented for fractures when a 2-D matrix is considered. . . . . . . . . . . . . . . . . . 22

4.4 A single fracture and matrix coexist in this 2 -D domain. . . . . . . . . . . . 23

4.5 Left, a schematic representation of how the vertices are set to calculate the basis function for coarse node $i$. Right, the result of these calculations. All values in this dual coarse grid are between 1 and 0 as can be seen on the scale bar on the far right. 24

4.6 Left, a schematic representation of how the vertices are set to calculate the basis function for coarse node $i$ in a dual coarse node containing two fractures. Right, the result of these calculations. Left of the lower fracture all grid cells have value zero as the low permeability of the fracture causes these cells to be cut off from the cells to the right of the fracture. . . . . . . . . . . . . . 
4.7 Left, a schematic representation of how the vertices are set to calculate the basis function for coarse node $i$ in an dual coarse node containing one fracture. Right, the result of these calculations. . . . . . . . . . . . . . . . .

4.8 This figure shows how different levels are used in a simulation. Notice how the front of the injected phase is surrounded by a finescale resolution and the coarser grid cells are around an area where no gradient in saturation is present. Around the wells (in every corner) a finescale grid is imposed. . . . . . . . . . . . . .

5.1 The fracture map used for the test cases mentioned below. Fractures with a high permeability are presented in red whereas fractures with a low permeability are presented in blue. . . . . . . . . . . . . . . . .

5.2 The figures a-e are a visualization of the single phase pressure solution. Here, e is the error as computed by equation $5.0 .1 \ldots \ldots \ldots \ldots$

5.3 (a) Fracture map with the black line being the trajectory over which the pressure is plotted. (b) The pressure decline belonging to the trajectory shown in (a). The trajectory length is dimensionless. . . . . . . . . . . . . . . .

5.4 The fracture map used for the test cases mentioned below. Fractures with a high permeability are presented in red whereas fractures with a low permeability are presented in blue.

5.5 The figures a-e are a visualization of the single phase pressure solution. Here, e is the error as computed by equation $5.0 .1 \ldots \ldots \ldots \ldots$

5.6 (a) Fracture map with the black line being the trajectory over which the pressure is plotted. (b) The pressure decline belonging to the trajectory shown in (a). The trajectory length is dimensionless.

5.7 The fracture map used for the test cases mentioned below. Fractures with a high permeability are presented in red whereas fractures with a low permeability are presented in blue. . . . . . . . . . . . . . . . .

5.8 The figures a-e are a visualization of the single phase pressure solution. Here, e is the error as computed by equation $5.0 .1 \ldots \ldots \ldots \ldots \ldots$

5.9 (a) Fracture map with the black line being the trajectory over which the pressure is plotted. (b) The pressure decline belonging to the trajectory shown in (a). The trajectory length is dimensionless. . . . . . . . . . . . . . . .

5.10 The fracture map used for the test cases mentioned below. Fractures with a high permeability are presented in red whereas fractures with a low permeability are presented in blue.

5.11 The figures a-e are a visualization of the single phase pressure solution. Here, e is the error as computed by equation $5.0 .1 \ldots \ldots \ldots \ldots$

5.12 (a) Fracture map with the black line being the trajectory over which the pressure is plotted. (b) The pressure decline belonging to the trajectory shown in (a). The trajectory length is dimensionless.

5.13 The fracture map used for the test cases mentioned below. Fractures with a high permeability are presented in red whereas fractures with a low permeability are presented in blue. . . . . . . . . . . . . . . . . .

5.14 The figures a-e are a visualization of the single phase pressure solution. Here, e is the error as computed by equation $5.0 .1 \ldots \ldots \ldots \ldots \ldots$

5.15 (a) Fracture map with the black line being the trajectory over which the pressure is plotted. (b) The pressure decline belonging to the trajectory shown in (a). The trajectory length is dimensionless. . . . . . . . . . . . . . . . . .

5.16 The figures a-e are a visualization of the single phase pressure solution. Here, e is the error as computed by equation $5.0 .1 \ldots \ldots \ldots \ldots \ldots$ 
5.17 (a) Fracture map with the black line being the trajectory over which the pressure is plotted. (b) The pressure decline belonging to the trajectory shown in (a). The trajectory length is dimensionless. . . . . . . . . . . . . . . .

5.18 The fracture map used for the test cases mentioned below. Fractures with a high permeability are presented in red whereas fractures with a low permeability are presented in blue.

5.19 The figures a-e are a visualization of the single phase pressure solution. . . . . . . .

5.20 (a) Fracture map with the black line being the trajectory over which the pressure is plotted. (b) The pressure decline belonging to the trajectory shown in (a). The trajectory length is dimensionless. . . . . . . . . . . . . . . . . .

5.21 The fracture map used for the test cases mentioned below. Fractures with a high permeability are presented in red whereas fractures with a low permeability are presented in blue. . . . . . . . . . . . . . . . . . 45

5.22 The figures a-e are a visualization of the single phase pressure solution. . . . . . . . 46

5.23 (a) Fracture map with the black line being the trajectory over which the pressure is plotted. (b) The pressure decline belonging to the trajectory shown in (a). The trajectory length is dimensionless. . . . . . . . . . . . . . . .

5.24 The fracture map used for the test case mentioned below. Fractures with a high permeability are presented in red whereas fractures with a low permeability are presented in blue.

5.25 The figures a-e are a visualization of the single phase pressure solution.

5.26 (a) Fracture map with the black line being the trajectory over which the pressure is plotted. (b) The pressure decline belonging to the trajectory shown in (a). The trajectory length is dimensionless.

5.27 The fracture map used for the test cases mentioned below. Fractures with a high permeability are presented in red whereas fractures with a low permeability are presented in blue.

5.28 The figures a-e are a visualization of the single phase pressure solution.

5.29 (a) Fracture map with the black line being the trajectory over which the pressure is plotted. (b) The pressure decline belonging to the trajectory shown in (a). The trajectory length is dimensionless.

5.30 A pressure solution for an MMs simulation with fractures given coarsening ratio of 3 and a matrix coarsening ratio of 3 with a pressure scale that has a range to account for all pressures in the solution (left). A visualization of the location of vertices, edges and faces of the matrix but also the location of the fractures (with their pressure solution) for the green box in which negative pressures are present (right).

5.31 A pressure solution for an MMs simulation with fractures given coarsening ratio of 3 and a matrix coarsening ratio of 9 with a pressure scale that has a range to account for all pressures in the solution (left). A visualization of the location of vertices, edges and faces of the matrix but also the location of the fractures (with their pressure solution) for the same green box as in figure 5.30 (right). . . . . . . .

6.1 The fracture map used for the test cases mentioned below. Fractures with a high permeability are presented in red where fractures with a low permeability are presented in blue. . . . . . . . . . . . . . . . . . . .

6.2 The figures b-e are a visualization of the reservoir after 150 days of injection and production. This is at time-step 75 as each time-step is set to be 2 days, with CR denoting coarsening ratio. . . . . . . . . . . . . . .

6.3 (a) Percentage of active grid cells with CR denoting coarsening ratio for fractures as the matrix coarsening ratio is kept equal throughout the simulations. (b) Saturation error $(\mathbf{c})$ Pressure error $\ldots \ldots \ldots \ldots \ldots \ldots$ 
6.4 The figures b-e are a visualization of the reservoir after 150 days of injection and production. . . . . . . . . . . . . . . . . . . 59

6.5 (a) Percentage of active grid cells (b) Saturation error (c) Pressure error . . . . . . 60

6.6 The figures b-e are a visualization of the reservoir after 150 days of injection and production. The gridding of the reservoir is plotted over the saturation solution to provide insight in how the front is captured by the grid refinement scheme. . . . . . 60

6.7 The figures b-e are a visualization of the multiphase pressure solution at time step 1 (i.e. 1 day of injection). To be able to compare the solutions clearly, the fractures have not been shown in the figures b-e. The fractures present can be seen in the fracture map, figure a . . . . . . . . . . . . . . . . 61

6.8 The figures $\mathbf{b} \& \mathbf{d}$ are a visualization of the multiphase pressure solution at time step 1 (i.e. 1 day of injection). To be able to compare the solutions clearly, the fractures have not been shown in the figures b-e. The fractures present can be seen in the fracture map, figure a \& c.

(a) Fracture map with the blue line being the trajectory over which the pressure is plotted. The fractures are not shown with their corresponding permeabilities as this differs for certain pressure declines. This plot only shows the location of fractures and the trajectory chosen over which to plot the pressure decline. (b) The pressure decline belonging to the trajectory shown in (a). The trajectory length is dimensionless. . . . . . . . . . . . . . . . . .

A.1 The Mohr-Coulomb plot used for the classification of the fracture set mentioned in

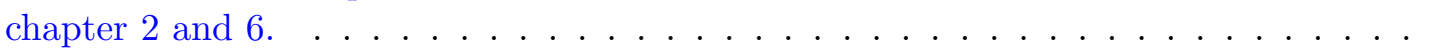

C.1 (a) Fracture map (b) Finescale pressure solution (c) Coarsening ratio 3 for fractures (d) Coarsening ratio 9 for fractures (e) Coarsening ratio 15 for fractures. The figures b-e are a visualization of the reservoir after 150 days of injection and production. . .

(a) Fracture map (b) Finescale pressure solution (c) Decoupled (d) Fully coupled (e) Highly permeable fractures coupled. The figures b-e are a visualization of the reservoir after 150 days of injection and production. . . . . . . . . . . . .

C.3 (a) Fracture map (b) Finescale pressure solution (c) Decoupled (d) Fully coupled (e) Highly permeable fractures coupled. The figures b-e are a visualization of the reservoir after 150 days of injection and production. The gridding of the reservoir is plotted over the pressure solution to provide insight in how the front is captured by the grid refinement scheme. . . . . . . . . . . . . . . . . 


\section{Chapter 1}

\section{Introduction}

Naturally fractured reservoirs (NFRs) are in the centre of attention due to their large reserves of geo-energy (e.g. hydrocarbon) worldwide [4] [5] [6]. However, when compared to conventional reservoirs, fractured reservoirs tend to show a more complex and heterogeneous character. The distribution and characteristics of fractures cannot be adequately sampled along a 1-D borehole and are mainly invisible on seismic images [7]. With these challenges in mind, NFRs are made up of two mediums, rock matrix and fractures. In general, hydrocarbons tend to be primarily stored in the rock matrix but the fractures play a substantial role in the flow and transport field. Understanding how these flow paths behave can enhance hydrocarbon recovery and play an important role when enhanced recovery methods are considered to further develop a naturally fractured reservoir.

Fractured reservoirs are not only of interest for the development of hydrocarbon reservoirs, or extraction of other geo-energy resources. They are also relevant for safe cyclic storage of green gas and permanent storage of greenhouse gases. Once these fractured reservoirs are depleted, they can be filled with e.g. $\mathrm{CO}_{2}$ to be stored safely underground. To be sure no gasses leak to the surface, the characteristics of fractures must be examined in detail, as the fractures may be a cause of flow and thus leakage.

The fractures are in both cases the cause for enhanced flow in the reservoir. Knowing how the fractures effect the flow is a crucial step towards a more reliable energy production and an environmentally friendly subsurface engineering. That is why this work aims to bridge a gap between geological research on fractures and reservoir simulation where a digital model is created inspired by nature.

\subsection{Fracture Characteristics}

The distribution of fractures is difficult to sample along 1-D boreholes and the fractures present in a reservoir are mostly invisible on seismic images due to their small size. This results in a situation in which engineers do not know where fractures are present or how fluid flow behaves due to their presence. To gain understanding of how reservoirs are fractured, geologists have studied outcrops on the earths surface. By studying outcrops, fracture maps are obtained. This is done by flying a drone over an outcropping pavement, interpreting the images made and digitizing the fractures visible on the pavements [7] [3] [8] [9].

Because no fracture map of the subsurface can be acquired, the fracture map of the surface has to be used as an analogy [7] [10] [11]. These fracture maps are only showing the distribution, orientation and length of the fractures. The characteristics influencing the flow of the fractures are still unknown at this point. Fractures can have flow enhancing properties when they have a relatively high permeability or they can cause flow to be blocked when they have a relatively low permeability. 
A meaningful simulation can only be performed once the characteristics of the fractured media are also meaningfully defined. In this work, we define the fracture network properties based on the principle stress, $\sigma_{1}$, minor principle stress, $\sigma_{3}$, and the orientation of $\sigma_{1}$ [12] [13] [14]. As such, the simulator populates the discrete fracture properties based on the geological information.

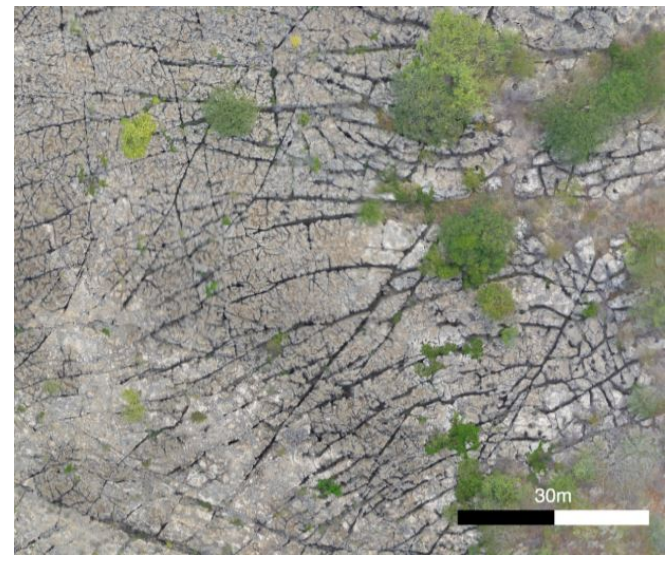

(a)

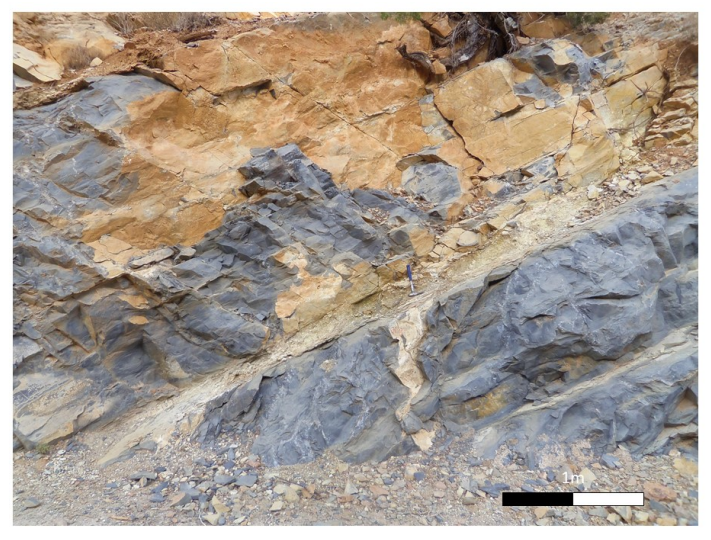

(b)

Figure 1.1: Fractures in geological formations at different scales. (a) Fractures visible from a drone image in Apodi, Brazil (photo taken by Kevin Bisdom [1]). (b) Fractures visible in an outcrop in the Western High Atlas, Morocco (photo taken by the author).

\subsection{Modeling Fractures}

To model flow through fractures accurately, multiple methods are used. One way of modeling the flow through fractured media is to upscale the fractures by averaging fracture permeability with matrix permeability. This results in a single representation of the porous media [2]. In case of high contrast between fracture and matrix, this method proves to be inaccurate as a high contrasting fracture with respect to the matrix is averaged out of the solution.

Besides this option, Direct Numerical Simulation (DNS) can be considered [15]. Using this method the grid is adapted to the fracture's geometry and resolution. Because the size of a subsurface reservoir is not comparable in size with the fracture aperture (kilometer scale versus millimeter scale) this option is not a practical option for life size reservoirs.

These issues have resulted in the development of an approach called Discrete Fracture Modeling (DFM). This approach is based on fractures being in a separate system in a lower dimensional space than that of the matrix. Through a transfer function the two systems are coupled. Because this method delivers more accurate results it has been subject to numerous studies (e.g. [16], [15] and [17]). In the following section a closer look is taken into the DFM approach.

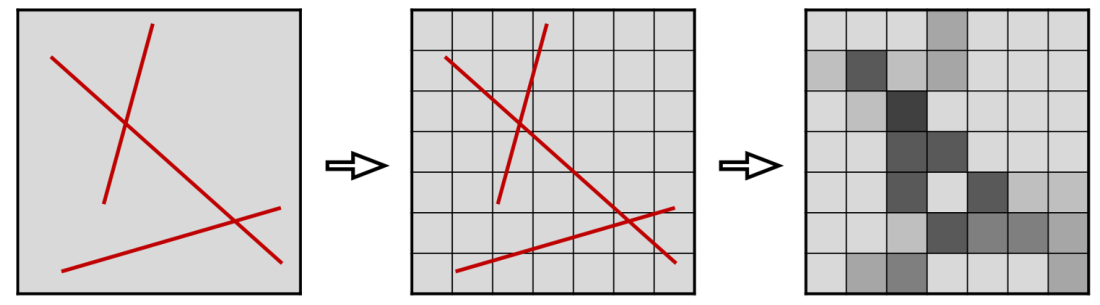

Figure 1.2: The concept of upscaling fractures visualized. In a fractured matrix a grid is imposed and where the fractures cross these grids the properties belonging to these grids are modified to adjust for the presence of fractures [2]. 


\subsection{Discrete Fracture Model (DFM)}

The DFM approach can be split into two main setups. The first being Conforming Discrete Fracture Modeling (CDFM). Here fractures are enclosed by an unstructured grid that are triangular in shape. For complex fracture networks, this setup is not wanted as the grid generation will lead to very large linear system due to the amount of grids needed to build the fractures explicitly in the matrix [15].

Other studies on the DFM approach have lead to the development of the Embedded Discrete Fracture Model (EDFM). This method is based on the discretization of fractures and matrix separately and using a flux transfer term to couple them [18]. The coupling of fractures by using a Connectivity Index (CI) is introduced by Hajibeygi et al. [19]. This research suggests using pEDFM, described by Tene et al. (2017), to consistently model fractures with a wide range of conductivity contrasts towards the matrix. This method introduces an extension to the EDFM appoach as the matrix and fracture cells in the vicinity of the fractures are modified [20].

\subsection{Multilevel Simulation Strategies}

It is challenging to simulate flow (pressure) and transport (saturation) occurring in the reservoir with such a resolution that all the complex processes can be captured. Examples of these complex processes are multiple fronts due to a highly heterogeneous reservoir and, for multiphase flow, the fingering at these fronts [21]. The size of a reservoir (order of magnitude of $\mathrm{km}$ ) is too big in comparison with the scale at which certain effects have to be captured (order of magnitude of $\mathrm{cm}$ ). To simulate these important components of the flow and transport occurring in the reservoir a very fine grid must be implemented.

However, such a model will turn out to be computationally expensive. To overcome this problem, methods have been developed to decrease the computational costs. This work makes use of two different approaches, the 'Static Multilevel Multiscale' (MMs) approach and the 'Algebraic Dynamic Multilevel' (ADM). Both methods have similar setups but can be used for different purposes. In chapter 4 the methods are described in more detail.

In the work proposed by S.M. Hosseini-Mehr [18] multiple levels of coarse grids are introduced (Multilevel Multiscale, MMs) and combined with a pEDFM approach. The ADM method as described in [21] also complies with fractures in a pEDFM system [22]. Both approaches reduce the amount of gridcells and decreases computational costs for large systems. This work develops both approaches to decrease computational costs further while keeping the accuracy of solution within a desirable range. The decreasing of computational costs is a necessary step to be able to simulate a reservoir with a geologically realistic fracture map.

\subsection{Research goals}

The goals of this research are presented in this section. From the introduction given it becomes clear that it is a tough challenge to incorporate realistic fractures in a meaningful reservoir simulation. Up to now no realistic fracture maps are being used in the Darsim research groups simulations. To be able to do this the first research goal is set to be the implementation of a method to incorporate a realistic fracture input to be used in reservoir simulations.

With regards to reservoir simulation this work aims to capture the effect of fractures with minimum computational efforts. With this in mind, the research aims to use geology \& characterization of 
fracture properties to build up the computational geo-model and develop a unified homogenizedexplicit fracture modeling approach for it.

Besides presenting this approach, this research illustrates the concept of flexible coarsening which is used in multiscale solutions. The flexible coarsening is implemented to capture fractures flexibly at different resolutions, depending on the fractures' characteristics. Finally, a field applicability study of the model is performed.

The outline of this thesis report is as follows:

First, chapter 2 describes how nature is studied by geologists and how this information is used to derive the properties belonging to fractures.

The method used to model the fractures and their properties are discussed in chapter 2 , is the pEDFM model. In chapter 3 governing equations and finescale discretization using pEDFM is discussed.

A description of both multiscale methods (MMs and ADM), including flexible coarsening, is given in chapter 4. Results belonging to the MMs and ADM approach are presented in chapter 5 and 6 respectively.

Finally a chapter on the conclusion and discussion of this work is presented in chapter 7 . 


\section{Chapter 2}

\section{Obtaining Realistic Fracture Maps}

As mentioned in chapter 1, the distribution and characteristics of fractures in a realistic naturally fractured reservoir are not easily determined. This means that geologists are looking for ways to gain a better understanding of how fractures are present in the subsurface. Outcrop studies on fracture pavements are being performed as they can be considered as an analogy to subsurface fracture sets [7]. To be able to acquire more information on the characteristics that influence flow, multiple studies have been performed. This research presents the link between fracture mapping and geomechanics with the DARSim reservoir simulation.

\subsection{Fracture maps}

The upper crust of the earth is very heterogeneous and outcrops such as studied by Kevin Bisdom [23] and Pierre Bruna [7] show this at first glance. The outcrops reveal numerous pre-existing weak planes. These fractures, joints and faults, represent anisotropy in potential reservoir formations. Fractures are present in many different scales. The size of fractures can range from a few millimeters to a couple of kilometers long. The fractures in the intermediate scale (at the order of tens to hundreds of meters), are often qualified and quantified using unmanned aerial vehicles (UAV's or drones). Studies on fracture presence performed in areas where rock formations of interest are present at the surface can be of great importance as the resulting fracture maps can be used as the fracture input for geologically meaningful simulations.

This study uses fracture maps that resulted from studies performed by Bisdom [23]. Fracture analysis was performed through drone images that have been merged into georeferenced orthomosaics and manually interpreted from which length, orientation and spatial distributions of the fractures were extracted.

Recent developments made in geological computing offer the chance to acquire fracture maps by a method called Multiple-Point Statistics. This method is introduced by P. Bruna [7]. Because the fracture maps made from outcrops are just an analogue for the subsurface and will never be a true representation of a subsurface fracture map, this method provides more analogues that are of geological relevance to a potential reservoir.

It is important to keep in mind that the fracture maps are not reality. The fact that the maps used are an analogue, tells us that this is the main source of error. No matter how precise the permeability of fractures is determined or how precise flow is computed in the remaining part of this research, the fact that the fracture map is not how the true fracture map is, makes all the other errors seem small. 


\subsection{Fracture Classification}

In section 2.1 a description is given on how fractures are being mapped from outcropping pavements to be used as analogue for subsurface fracture systems. Section 2.2 gives more insight in how the mapped fractures can be classified as conductive fractures (i.e. high permeability when compared to surrounding matrix) or as blocking fractures (low permeability when compared to surrounding matrix).

The characteristics of fractures can be hard to determine. This is due to the same reasons as why it can be hard to map the fractures. The fractures in the subsurface are invisible for researchers to determine their flow properties. In the past multiple researchers have tried to determine flow characteristics of fractures. Methods used in past research include determining the fracture aperture where aperture follows power law frequency scaling [24] [25].

A different method to predict fracture aperture is based on linear elastic fracture mechanics. Lawn and Wilshaw [26] introduced this method where aperture is based on the Poisson's ratio and Young's modulus, fracture length and $\mathrm{K}_{\mathrm{f}}$, so called fracture toughness [26] [27] [28].

The described approaches capture the fracture aperture, however this is not directly representative of the actual flow through the fracture network [29]. Research performed by Barton et al. [14] and Zoback [12] [30] introduce the concept of hydraulically open fractures. In this research the Coulomb friction criterion is considered and used as a guide which fractures are hydraulically open. This Coulomb friction criterion is further discussed in the following subsection.

\subsubsection{Coulomb Friction Criterion}

A set of linear equations form the conditions for which materials, such as a rock matrix, will fail. This is considered to be the Mohr-Coulomb criterion. The effect of the intermediate principle stress, $\sigma_{2}$, is neglected in this criterion [31]. The Mohr-Coulomb criterion can be written as a function of normal stress, $\sigma_{n}$ and shear stress, $\tau$ on the failure plane [32]. Coulomb's condition is based on a linear failure envelope to determine the critical combination of $\tau$ and $\sigma$ that will cause failure on some plane [31]. The formula used for the Coulomb criterion for this linear failure envelope for preexisting planes is [13]:

$$
\tau=\mu \sigma_{n}
$$

where $\mu$ is the coefficient of static friction.

The little cohesion the fractures have, causes the coefficient of static friction on the plane, hereafter referred to as $\mu$, to be different than the coefficient of internal friction. Thus, the failure envelope in a Mohr-Coulomb plot describing the failure of rock, will be different for pre-existing fractures [13].

James Byerlee performed research to show, to a first order, $\mu$ is independent of rock type [33]. The research summarizes a large amount of experimental data on rock friction. This has become known as 'Byerlee's Law' even though it is based on an empirical relationship. From this research, Mark Zoback [12] and Richard Allmendinger [13] use $\mu=0.6$. Using this value,

where, $\tau$ and $\sigma_{n}$ are the shear and normall stress respectively.

Figure 2.1 shows an envelope for failure along pre-existing planes. The crossing of the pre-existing failure envelope does not mean that failure will actually occur. The intersections define a range of orientations in which the pre-existing weak planes must lie to be considered hydraulically active. In figure 2.1 the angles $2 \beta_{1}$ and $2 \beta_{2}$ are shown. The angles $\beta_{1}$ and $\beta_{2}$ are the boundaries of the 


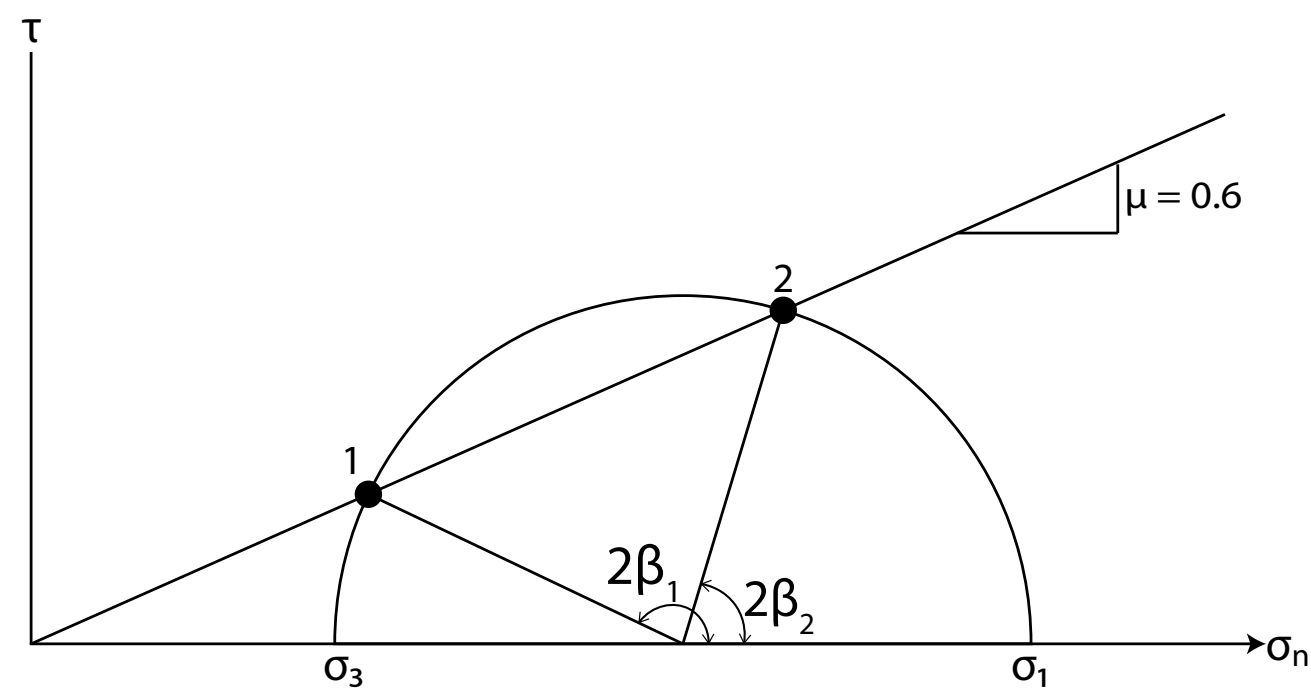

Figure 2.1: Mohr's circle for stress and failure envelopes. The coefficient of static friction on preexisting planes with a value of $\mu=0.6$ and the principle stresses, $\sigma_{1}$ and $\sigma_{3}$ are plotted to define the range of $\beta_{1}$ to $\beta_{2}$ in which fractures will be critically stressed.

range for failure.

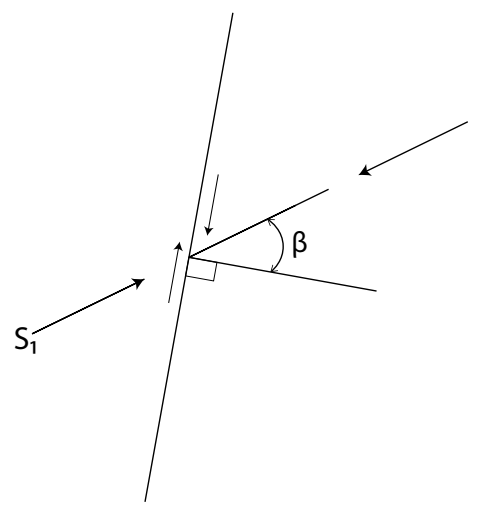

Figure 2.2: A fracture shown that is optimally orientated for frictional sliding in a 2-dimensional field.

In figure 2.2 the angle $\beta$ is the angle between the fault normal and $\sigma_{1}$. If this angle $\beta$ lies within the range of $\beta_{1}$ and $\beta_{2}$, the fracture can be considered critically stressed, or hydraulically active as it lies within the range of failure [13] [12] [14]. This is then used to get insight into the permeability characteristics of the fracture. Fractures that are considered to be critically stressed are given a high permeability with respect to the surrounding matrix whereas the fractures that are not considered critically stressed are given a low permeability with respect to the surrounding matrix.

Throughout geological time it is common for formations to experience different tectonic regimes. This leads to fractures present in a formation which are formed at different times and are formed at different orientations [12]. This explains the variety of orientations that can be present in a fracture map. 
In this research a geological interpretation made by Kevin Bisdom [3] is used to perform this approach on. After digitizing fractures based on drone footage, the fracture location and stress dependent permeability are implemented to use as a field scale test case. The result of this approach can be seen in figure 2.3 .

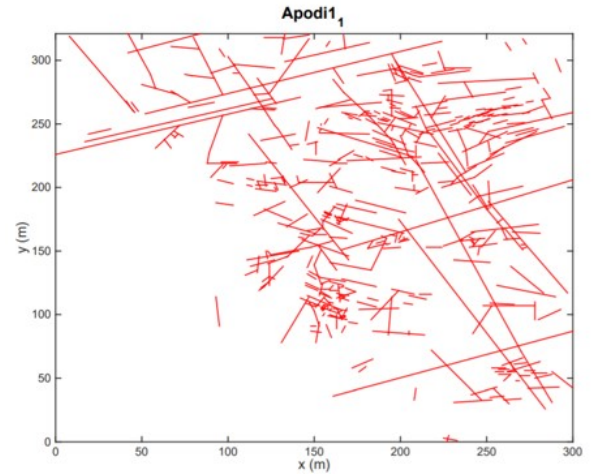

(a)

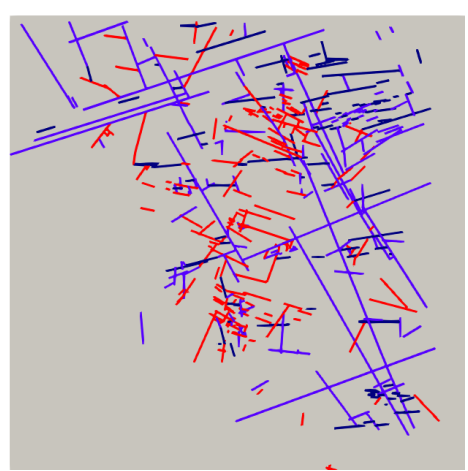

(b)

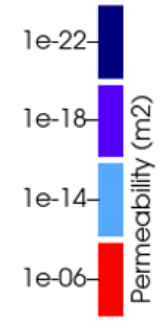

Figure 2.3: (a) The location of the fractures as interpreted by Kevin Bisdom [3] (b) The fractures with an assigned permeability

In figure 2.3 all fractures shown left are processed through the methodology described in this chapter and are either assigned a high or a low permeability. The fractures with a low permeability are divided in three different categories. They are considered hydraulically inactive but this can be a broad range of permeabilities. If a fracture just falls out of the range to be considered hydraulically stressed, the fracture is assigned a permeability equal to the matrix. For fractures that just fall out of this range, fractures are given a permeability of $10^{-18} \mathrm{~m}^{2}$. If a fracture even falls out of this range, it is considered to be a blocking fracture and is assigned a permeability of $10^{-22} \mathrm{~m}^{2}$. In table 2.1 the classification can be found.

Table 2.1: Fracture permeability classification based on the angle $\beta$. Whenever a fracture's angle $\beta$ falls within a range, the corresponding permeability is assigned to it.

\begin{tabular}{|l|l|l|l|l|}
\hline Fracture angle $\beta$ & Range 1 & Range $1+10^{\circ}$ & Range $1+20^{\circ}$ & $>$ Range $1+20^{\circ}$ \\
\hline Fracture Permeability $\left(\mathrm{m}^{2}\right)$ & $10^{-6}$ & $10^{-14}$ & $10^{-18}$ & $10^{-22}$ \\
\hline
\end{tabular}

This results in the fracture map shown right. The map consists of 447 fractures and a north-south direction is assumed for $\sigma_{1}$ [23]. The Mohr-Coulomb plot belonging to this test case is shown in appendix A.

It should be clear that in previous research performed by the DARSim research group there was no indication of which fracture was conductive and which fracture was a blocking fracture. Therefor the methodology proposed in this chapter should be seen as a rough estimate of fracture conductivity and as first step to realistic stress dependant fracture permeability. Besides fracture conductivity, fracture placement was not based on geology. Still, using this method a fracture map is used in which the location of fractures is derived from surface pavements but it gives an impression of how a real fracture map can geologically look like. 


\section{Chapter 3}

\section{Solving strategy for EDFM method}

With a fracture map that is ready to be used according to the approach described in chapter 2, the fracture and matrix need to be discretized and a strategy needs to be implemented to accurately simulate flow.

In this chapter the use of a Fully Implicit (FIM) solver simulation will be presented as implemented by Hajibeygi et al [21]. A FIM scheme is used to obtain a coupled discrete system with two main unknowns (i.e. pressure and saturation). The fractures in the reservoir are represented by the implementation of the Embedded Discrete Fracture Model (EDFM) method.

\subsection{EDFM Concept}

The concept of EDFM is to calculate the main unknowns (i.e. pressure and saturation) in fractures separately from the matrix. Through a transfer function they are coupled and the system is brought together again. This gives the fractures and matrix an independent character and makes computation of the unknown easier.

The fractures, being very thin sheets throughout the reservoir, are characterized as a lower dimensional object. This work presents reservoirs in a 2-D representation, and therefor fractures are represented as 1-D lines. The concept is visualized in figure 3.1

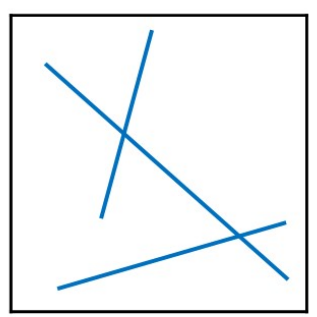

(a)

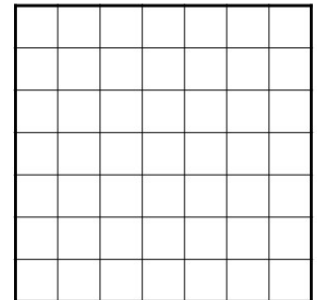

(b.1)

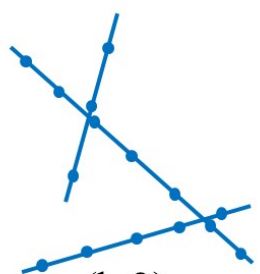

(b.2)

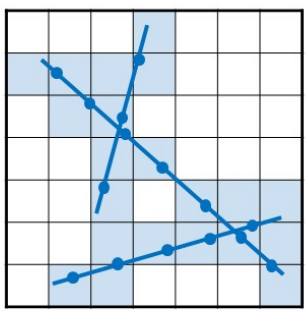

(c)

Figure 3.1: The concept of EDFM visualized. In figure a) the matrix and 3 fractures can be seen. This matrix is discretized with a uniform, structured grid which can be seen in b.1). The fractures are also discretized but separately in figure b.2). After coupling the matrix and fractures through a transfer function they are brought together in c). 


\subsection{Governing Equations}

In this section the governing equations are presented for a single- and multiphase incompressible flow. In the simulations no gravity is taken into consideration. To obtain pressure globally throughout the reservoir the following mass balance equation is used.

$$
\frac{\partial\left(\phi \rho_{\alpha} S_{\alpha}\right)}{\partial t}-\nabla \cdot\left(\rho_{\alpha} \lambda_{\alpha} \cdot \nabla p\right)=\rho_{\alpha} q_{\alpha}
$$

The phase mobility, $\lambda$, in the equation above can be written as $\lambda_{\alpha}=\frac{k_{r \alpha}}{\mu_{\alpha}} K=f_{\alpha} \lambda_{t}$.

In equation 3.2.1 $\phi$ is porosity, $\rho$ is density and $S$ is saturation of phase $\alpha$. Moreover, $p$ is the pressure. In the mobility equation, note that $k_{r \alpha}$ and $\mu_{\alpha}$ are the relative permeability and viscosity of phase $\alpha$ and $K$ is the absolute rock permeability.

Incompressible fluid and rock is assumed and this leads to $\rho_{\alpha}$ and $\phi$ are constant over time. This leads to the following equation:

$$
\phi \frac{\partial S_{\alpha}}{\partial t}+\nabla\left(f_{\alpha}\left(-\lambda_{t} \cdot p\right)\right)=q_{\alpha}
$$

In equation 3.2.2, a mass balance equation is setup with incompressible fluid and rock assumed. The total mobility, $\lambda_{t}$, in combination with phase fractional flow, $f_{\alpha}$. The saturation constraint of the summation of all phases to add up to one, is essential in the mass balance equation.

In this section, 3.2, the governing equations are discussed for both the matrix and the fractures. The approach of Embedded Discrete Fracture Modeling, EDFM, is designed to calculate pressure and saturation in the matrix and fractures separately. This means an independent matrix grid is set up, together with an independent fracture grid. In EDFM the connectivity between fractures and matrix are defined through flux interactions, $\Psi^{m f}$ and $\Psi^{f m}$. They are added to the EDFM pressure equation as follows:

$$
\phi \frac{\partial S_{\alpha}}{\partial t}+\nabla\left(f_{\alpha}\left(-\lambda_{t} \cdot p\right)\right)^{m}=q_{t}^{m}+\Psi^{m f}
$$

and

$$
\phi \frac{\partial S_{\alpha}}{\partial t}+\nabla\left(f_{\alpha}\left(-\lambda_{t} \cdot p\right)\right)^{f}=q_{t}^{f}+\Psi^{f m}
$$

for matrix and fractures respectively. Here $q^{m}$ and $q^{f}$ are the source terms on the matrix, $m$ and fracture, $f$. They are obtained by using the Peaceman well model [34]:

$$
q^{m}=\frac{P I \cdot \lambda_{t} \cdot\left(p^{w}-p^{m}\right)}{\Delta V}
$$

for the matrix and for fractures:

$$
q^{f}=\frac{P I \cdot \lambda_{t} \cdot\left(p^{w}-p^{f}\right)}{\Delta A}
$$

In the above equations $P I$ denotes the well productivity index, $\lambda_{t}$ the effective mobility between the well and the penetrated grid cell. The control volumes $\Delta V$ and $\Delta A$ are used in the discrete system for the matrix, $m$ and fracture, $f$, respectively.

The connectivities between fractures and matrix are defined through $\Psi^{m f}$ and $\Psi^{f m}$ :

$$
\Psi^{m f}=T_{m f}\left(p^{f}-p^{m}\right)
$$


and

$$
\Psi^{f m}=T_{f m}\left(p^{m}-p^{f}\right)
$$

In section 3.3 the transmissibility terms, $T_{m f}$ and $T_{f m}$ will be discussed.

\subsection{Connectivity Index}

The flux interactions, $\Psi^{m f}$ and $\Psi^{f m}$, can be written as follows:

$$
\begin{aligned}
& \Psi^{m f}=C I^{m f} \cdot \lambda_{t} \cdot\left(p^{f}-p^{m}\right) \\
& \Psi^{f m}=C I^{f m} \cdot \lambda_{t} \cdot\left(p^{m}-p^{f}\right)
\end{aligned}
$$

For two fractures which crossing each other, a separate flux interaction $\Psi^{f f}$ is implemented. This is for fracture-fracture connectivity.

$$
\Psi^{f_{i} f_{j}}=C I^{f_{i} f_{j}} \cdot \lambda_{t} \cdot\left(p^{f_{j}}-p^{f_{i}}\right)
$$

Here, CI denotes the connectivity index between two non-neighboring elements. It is defined as the area fraction of fracture element $k$ in matrix cel $(i, j), A_{i j, k}$, divided by the distance $\langle d\rangle_{i j, k}$ between fracture element $k$ and matrix cell $(i, j)$.

$$
C I_{i j, k}=\frac{A_{i j, k}}{\langle d\rangle_{i j, k}} \text { with }\langle d\rangle_{i j, k}=\frac{\int_{V_{i} j} x_{n}\left(\mathbf{x}^{\prime}\right) d \mathbf{x}^{\prime}}{V_{i j}}
$$

Due to the complexity of the implementation of the geometrical position of the fractures in a realistic fracture set and to simplify the extension of the integral to calculate $\langle d\rangle$, a numerical integral is used to calculate the average distance $\langle d\rangle_{i j, k}$ between fracture element $k$ and matrix cell $(i, j)$. This is done using equidistant points.

The area fraction of the corresponding fracture element $k$ in matrix cell $(i, j)$ is the lateral area of the plate that is located inside the matrix cell. Two examples are presented in 3.2. 


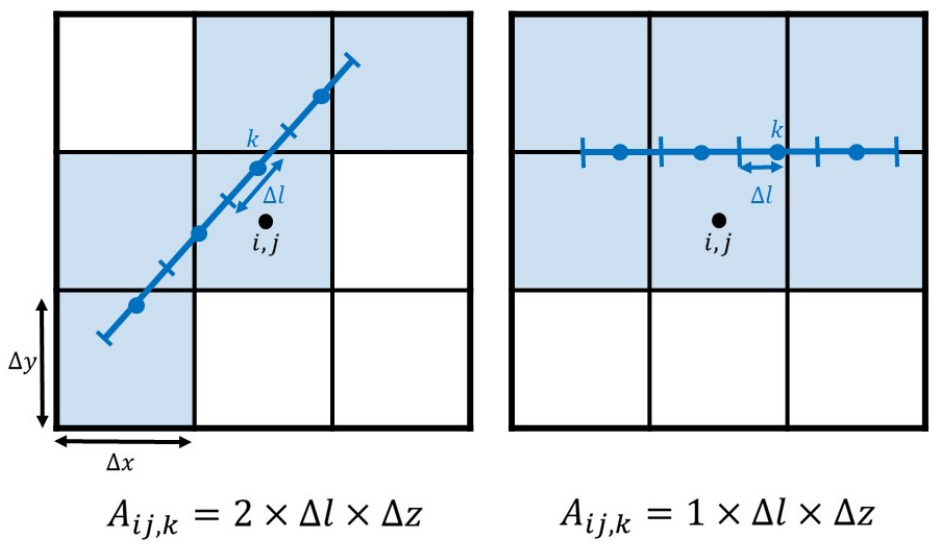

Figure 3.2: Examples of calculating the area fraction of the corresponding fracture element $k$ in matrix cell $(i, j)$.

The $A_{i j, k}$ in the left example is multiplied by 2 as the fracture has both faces cutting through the cell. The $A_{i j, k}$ in the right example only touches the boundary of the matrix cell $(i, j)$ and is therefor only multiplied by 1 as a single face is touching the matrix cell $(i, j)$. In figure 3.2 the $\Delta z$ is not visible in the sketch as it is a two-dimensional sketch. In a three-dimensional sketch $\Delta z$ would be seen as the length of the face intersecting matrix cell $(i, j)$ in the third dimension.

\subsection{Intersecting Fractures}

The fracture transmissibilities between elements of the same fracture are relatively simple to compute. However, when an intersection between fractures occurs, four fracture elements have a connection. To solve this issue a so-called star-delta transformation is used [15]. The transmissibility between the two fracture elements $(k$ and $l$ ) is written according to the following formula:

$$
T^{l, m} \simeq \frac{\alpha_{l} \alpha_{m}}{\sum_{k=1}^{n} \alpha_{k}} \text { where } \alpha_{k}=\frac{A_{f}^{k} \lambda_{t}^{k}}{\frac{1}{2} L^{k}}
$$

In figure 3.3 two fractures intersect. To calculate the transmissibility between the two fractures the can be obtained as:

$$
T^{1,2} \simeq \frac{\alpha_{1} \alpha_{2}}{\alpha_{1}+\alpha_{2}+\alpha_{3}+\alpha_{4}}
$$




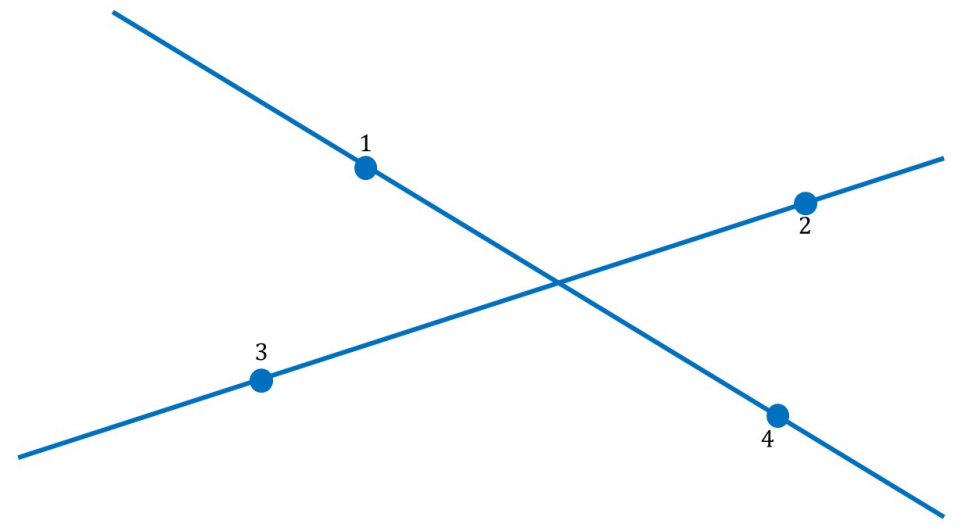

Figure 3.3: An intersection of two fractures. The blue dots represent the cell centers of the fractures.

\section{5. pEDFM Implementation}

When the permeability property of fractures range from highly conductive to blocking (i.e. high permeability relative to the surrounding matrix or low permeability relative to the surrounding matrix respectively), the EDFM implementation has its limitations. To correct for these limitations, the matrix-matrix, fracture-matrix and fracture-fracture connectivities are modified in the overlapping regions. The pEDFM method is implemented as described by M. Tene et al. [20].

Each transmissibility term, $T_{m f}$ and $T_{f m}$, is modified by multiplication of coefficient $\alpha$. This $\alpha$ is obtained by a series of calculations that are described below.

The first step is to detect all the matrix cells that are disconnected by an overlapping fracture. This is done as shown in figure 3.4 where the red line shows the interfaces of matrix cells that are overlapped by the fracture, shown in blue.

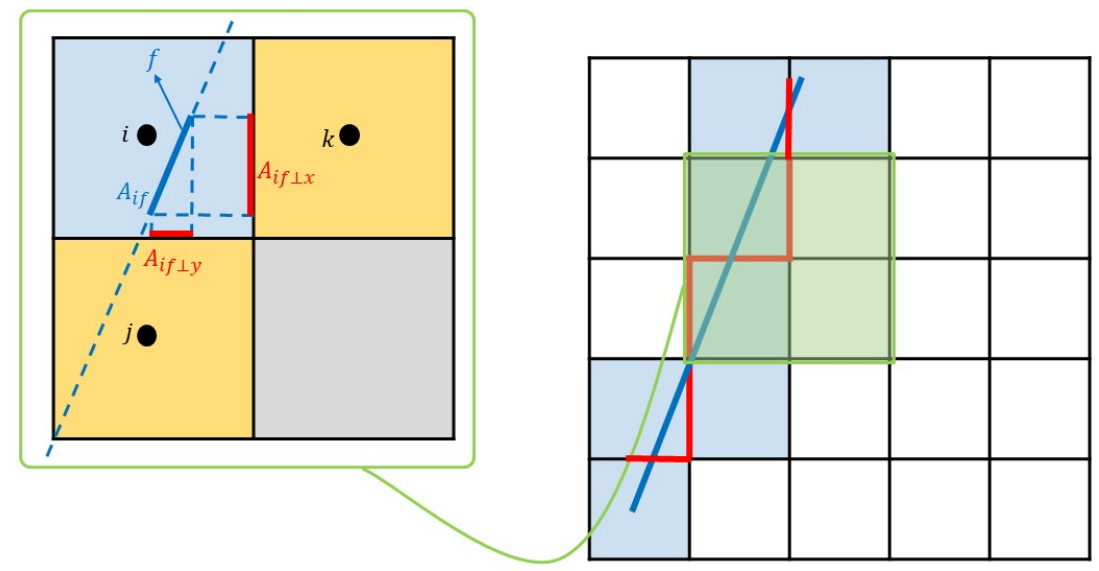

Figure 3.4: Illustration of the pEDFM method. The matrix cells of this $2 \mathrm{D}$ structured grid containing a single 1D fracture that are overlapped by the fracture are shown in blue. These are directly connected, as defined in classic EDFM. The yellow cells in the zoomed in view are the cells that take part in the additional non-neighboring connections between fracture and matrix grid cells. 
Fracture element $f$ overlaps grid cell $i$ as in figure 3.4. The fracture element has area fraction $A_{i f}$. The cells in yellow show a set of projections defined on the interface between the overlapped matrix grid cell $i$. The projection area fraction is obtained through the following:

$$
A_{i f \perp x_{e}}=A_{i f} \times \cos (\gamma), \quad x_{e} \in\{x, y, z\}
$$

The $\gamma$ in this calculation is the angle between the fracture element and the interface connecting the matrix grid cell $i$ and the neighboring grid cell in the corresponding dimension. The new transmissibilities are defined to connect the fracture element $f$ to each non-neighboring matirx grid cell. In the case of figure $3.4 j$ and $k$.

$$
T_{i_{e} f}=\frac{A_{i f \perp x_{e}}}{\langle d\rangle} \lambda_{t f}, \quad x_{e} \in\{x, y, z\}
$$

Still with $\langle d\rangle$ being:

$$
\langle d\rangle_{i j, k}=\frac{\int_{V_{i j}} x_{n}\left(\mathbf{x}^{\prime}\right) d \mathbf{x}^{\prime}}{V_{i j}}
$$

Here, $\lambda_{t f}$ is the effective fluid mobility between these cells. As a result of these new transmissibilities, the connectivity between the matrix grid cell $i$ and its corresponding neighboring cells is now:

$$
T_{i i_{e}}=\frac{A_{i i_{e}}-A_{i f \perp x_{e}}}{\Delta x_{e}} \lambda_{t f} \quad x \in\{x, y, z\}
$$

For simplicity of the calculations, the original transmissibility is multiplied by a factor $\alpha$. This factor is calculated as follows:

$$
\alpha=\frac{A_{i i_{e}}}{A_{i f \perp x_{e}}} x \in\{x, y, z\}
$$

With this fraction, $\alpha$ will be 1.0 for all cells that are fully overlapped by a fracture. This results in the new transmissibility to be zero for most cases (i.e. $T_{i i_{e}}=0$ ). This causes the parallel transmissibility to completely disappear and letting flux only occur on one route, first the matrix cell $i$, then the fracture and only then the matrix cell $j$ or $k$.

It is important to note that only fracture segments that intersect two cell centers, will have an effect on the transmissibility. In figure 3.4 the fracture cuts through the cell center of matrix cell $i$ and $k$ and causes the transmissbility to alter. The same goes for cell centers $i$ and $j$. Fracture $f$ has no influence to the transmissibility of the left cell neighbor of matrix cell $i$ as it doesn't cut through these two cell centers.

\subsection{Fully Implicit Discretization}

Using the saturation constraint and equation 3.2.2, fully implicit discretization of these equations are obtained. This is possible once all the pressure-dependent and saturation-dependent terms are calculated implicitly. Setting the residual (given in equation 3.6.1) equal to zero gives the solution at time step $(n+1)$.

$$
r_{\alpha}^{n+1}=\frac{\phi}{\Delta t}\left(S_{\alpha}^{n+1}-S_{\alpha}^{n}\right)-\nabla \cdot\left(\lambda_{\alpha}^{n+1} \cdot \nabla p^{n+1}\right)-q_{\alpha}^{n+1}=0
$$


Equation 3.6.1 is a non-linear function and needs to be solved by using a Newton-Raphson method. This gives the following linearization:

$$
r^{n+1} \approx r^{v+1}=r^{v}+\left.\frac{\partial r}{\partial p}\right|^{v} \delta p^{v+1}+\left.\sum_{i=1}^{N_{p h}-1} \frac{\partial r}{\partial S_{i}}\right|^{v} \delta S_{i}^{v+1}
$$

where, $v$ represents the iteration step and $N_{p h}$ the total number of phases. At each Newton Raphson iteration, the system of equation is written as $\boldsymbol{J}^{v} \delta x^{v+1}=-r^{v}$. Here, the $\boldsymbol{J}^{v}$ is the Jacobian matrix including all the derivatives. The $\delta x$ is the vector of unknowns. For a two-phase system consisting of oil $(o)$ and water $(w)$ with pressure $(p)$ and saturation $(S)$ with main unknowns, the linear system would read:

$$
\left(\begin{array}{ll}
J_{w p} & J_{w S} \\
J_{o p} & J_{o S}
\end{array}\right)\left(\begin{array}{l}
\delta p^{v+1} \\
\delta S_{w}^{v+1}
\end{array}\right)=-\left(\begin{array}{l}
r_{w}^{v} \\
r_{o}^{v}
\end{array}\right)
$$

In the equation 3.6.3 the rows belong to phase $\alpha$ and consist of a block representing pressure $(p)$ and saturation $(S)$ respectively. To compute pressure and saturation for a natural field-scale reservoir the FIM simulation can be computationally too expensive for a fine-scale grid. This is why in chapter 4 multiscale is presented. Here the issue of reducing computational costs is further investigated. 


\section{Chapter 4}

\section{Multiscale Methods for Flow and Pressure}

\subsection{Multilevel Multiscale (MMs)}

In this chapter, the multiscale approach is defined. First, the Multilevel Multiscale (MMs) approach is discussed. The method is described in general and can be used for multiphase pressure and saturation solutions but in this work the MMs approach is used for obtaining a pressure solution for a single phase simulation. A single phase pressure solution is time independent which can be seen if equation 3.2.2 is rewritten for a single phase and with the saturation constraint in mind.

\subsection{Implementation Strategy}

This method is implemented to reduce the computational costs of running a detailed simulation of a true size reservoir. To capture complex physics, a finescale grid of $N_{f}=N_{f x} \times N_{f y}$ is considered good enough. For a three-dimensional case $N_{f z}$ is also considered.

The first step of using a multiscale grid is imposing the $N_{m}^{l}$ and $N_{f_{i}}^{l}$ grids on the matrix, $m$, and fracture, $f_{i}$. These grids are hierarchically nested and $l$ corresponds to the level of coarsening of the corresponding Cartesian grid. Besides this it is important to note that the finescale grid is equal to $l=0$. The fractures can be coarsened up to a different level than the matrix grid, providing flexibility for the user. While using MMs in this work the system in its most coarse configuration. This makes the simulation static and can therefor also be called a 'static Multiscale Multilevel' simulation.

To map solutions from finescale to the created multiscale grid (and vice versa) the restriction $(R)$ and prolongation operator $(P)$ are used. These operators are computed one time, at the very beginning of the simulation.

Using the $R$ and $P$ operators, the ADM system is written as follows:

$$
\boldsymbol{R}_{l}^{l-1} \ldots \boldsymbol{R}_{1}^{0} \boldsymbol{J}_{0} \boldsymbol{P}_{0}^{1} \ldots \boldsymbol{P}_{l-1}^{l} \delta \hat{x}_{A D M}=-\boldsymbol{R}_{l}^{l-1} \ldots \boldsymbol{R}_{1}^{0} r_{0}
$$

In equation 4.2.1 $\boldsymbol{R}^{l-1}$ is the restriction operator which maps the solution vector, $\delta \hat{x}_{A D M}$, at level $l-1$ to level $l$. $\boldsymbol{P}_{l-1}^{l}$ is the prolongation operator which maps part of the solution vector from level $l$ to level $l-1$. Once the multiscale system is solved, the solution for the coarser grid $\left(\delta \hat{x}_{A D M}\right)$ is prolonged on the finescale resolution. This results into an approximate solution $\left(\delta x_{0}^{\prime}\right)$ when 
compared with the solution that is obtained when only a finescale grid is used.

$$
\delta x_{0} \approx \delta x_{0}^{\prime}=\boldsymbol{P}_{0}^{1} \ldots \boldsymbol{P}_{l-1}^{l} \delta x_{A D M}
$$

In equation $4.2 .2, \delta x_{0}$ represents the finescale solution. Even though not the entire reservoir domain is in at multiscale level $l$, the prolongation operator $\boldsymbol{P}$ is obtained at each timestep for the entire domain. This becomes clear from equation 4.2.1. The prolongation operator is structured with two main diagonal blocks for the two main unknowns, saturation $(S)$ and pressure $(p)$.

$$
\boldsymbol{P}_{l-1}^{l}=\left(\begin{array}{cccc}
{\left[\left(P_{p}\right)_{l-1}^{1}\right]_{m m}} & {\left[\left(P_{p}\right)_{l-1}^{1}\right]_{m f}} & 0 & 0 \\
{\left[\left(P_{p}\right)_{l-1}^{1}\right]_{f m}} & {\left[\left(P_{p}\right)_{l-1}^{1}\right]_{f f}} & 0 & 0 \\
0 & 0 & {\left[\left(P_{S}\right)_{l-1}^{1}\right]_{m m}} & {\left[\left(P_{S}\right)_{l-1}^{1}\right]_{m f}} \\
0 & 0 & {\left[\left(P_{S}\right)_{l-1}^{1}\right]_{f m}} & {\left[\left(P_{S}\right)_{l-1}^{1}\right]_{f f}}
\end{array}\right)
$$

The restriction operator $\boldsymbol{R}$ is constructed similarly:

$$
\boldsymbol{R}_{l}^{l-1}=\left(\begin{array}{cccc}
{\left[R_{l}^{l-1}\right]_{m}} & 0 & 0 & 0 \\
0 & {\left[R_{l}^{l-1}\right]_{f}} & 0 & 0 \\
0 & 0 & {\left[R_{l}^{l-1}\right]_{m}} & \\
0 & 0 & & {\left[R_{l}^{l-1}\right]_{f}}
\end{array}\right)
$$

To maintain mass conservation as convergence is reached on the multilevel grid, a finite-volume restriction operator is used:

$$
R_{l}^{i-1}(s, t)= \begin{cases}1 & \text { if cell } \mathrm{s} \text { is inside coarser cell } \mathrm{t}\{s, i\} \in E \\ 0 & \text { otherwise }\end{cases}
$$

The prolongation operator for pressure, $P_{p}$, uses multilevel multiscale basis functions as interpolator (see 4.3). The prolongation operator for saturation, $P_{S}$, uses a transposed restriction operator, $P_{S}=R^{T}$. This results in a prolongation operator, $P_{S}$, to be filled with either ones or zeroes.

This is because the pressure is a global property of the reservoir whereas the saturation is a local solution.

\subsection{Basis functions for Multiscale}

As can be seen in section 4.2 the restriction operator in equation 4.2.4 is filled with either ones or zeroes. The prolongation operator however is filled with so called basis functions. This section gives a closer look in how the basis functions fill up the prolongation operator for pressure.

\subsubsection{Coarse Scale Grids}

First, the finescale pressure system of equations is assembled. Once this is performed, the coarse grids are projected on the fine scale grid cells for fractures and matrix.

To ensure the coarse grid nodes are located on the corners and edges of the general domain, the number of fine grid cells must be chosen according to the following equation for a 2D-domain:

$$
\left(N_{c}-1\right) \cdot \gamma^{l}+1=N_{f}
$$




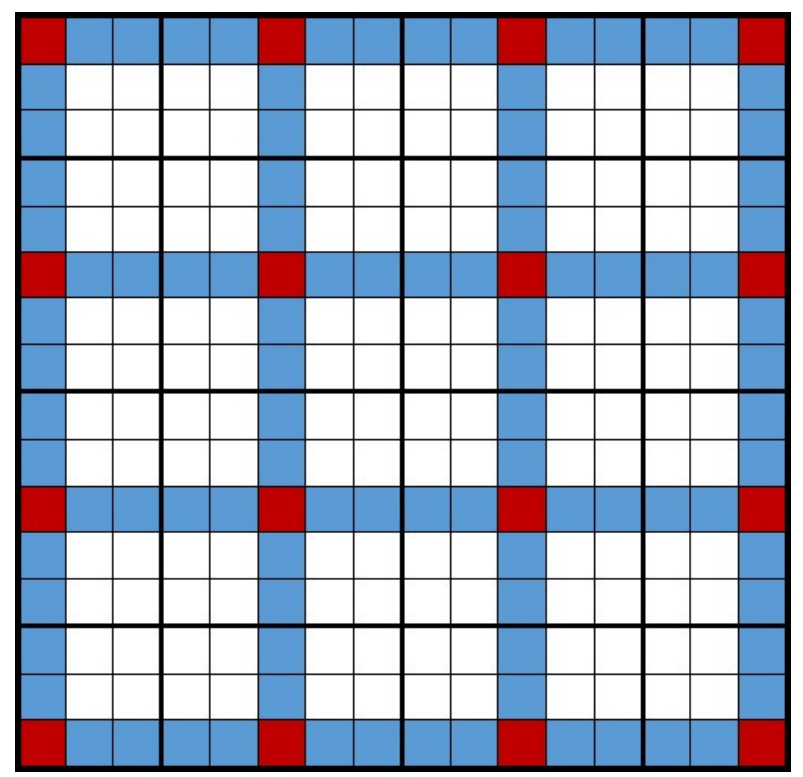

Figure 4.1: Here a coarse grid is projected on a fine scale grid. Using the formula 4.3.1: $\left(N_{c}-1\right) \cdot \gamma^{l}+1=(4-1) \cdot 5^{1}+1=16$. So 16 grids in both $\mathrm{x}$ and $\mathrm{y}$ direction are used.

where, $N_{c}$ is the number of coarse grid nodes (in either $x$ or $y$ direction), $l$ is the level of multiscale, $\gamma$ the coarsening ratio and $N_{f}$ the number of finescale grids (in the chosen direction $x$ or $y$ ).

In figure 4.1 it is shown how a fine scale grid consisting of 16x16 grid cells is represented in a coarser grid that consists of $3 \times 3$ dual coarse grids. A dual coarse grid consists of four coarse grid nodes at corners, in figure 4.1 these coarse grid nodes are shown in red. They are also referred to as vertices. The blue grids are referred to as edges, as they are the edges that make up the dual coarse grids. The remaining white cells are referred to as faces.

This is not the only configuration of vertices that is a possibility when it comes to the projection of coarse scale grids. The placement of the vertices on the corners is an approach which has been implemented more recently. Before the implementation of vertices on the corner, vertices were placed in a different manner. Using the formula 4.3.2, the vertices are placed as can be seen in figure $4.2[35]$.

$$
N_{c} \cdot \gamma^{l}=N_{f}
$$




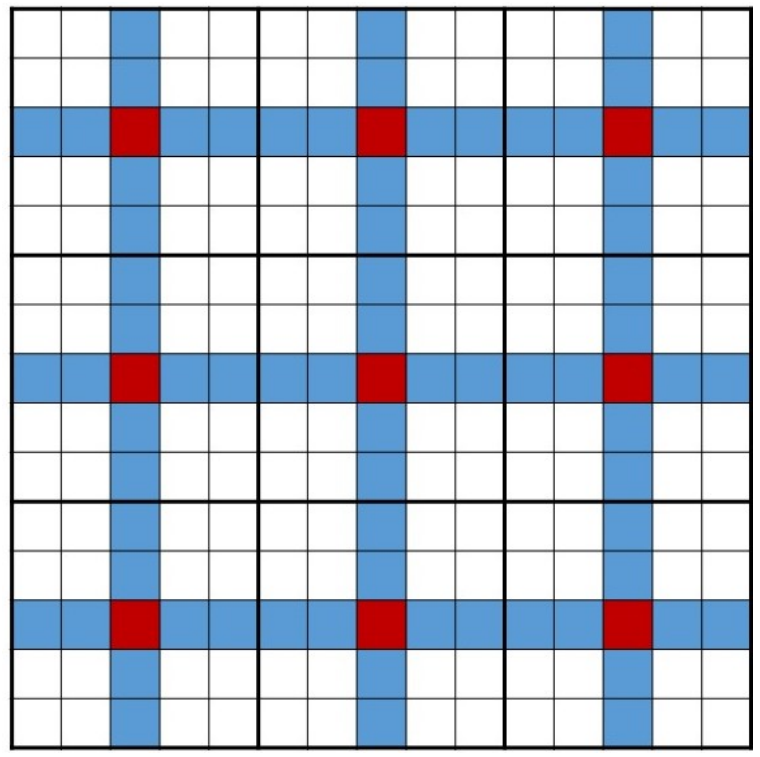

Figure 4.2: Here a coarse grid is projected on a fine scale grid. Using the formula 4.3.2: $N_{c} \cdot \gamma^{l}=(3) \cdot 5^{1}+1=15$. So 15 grids in both $\mathrm{x}$ and $\mathrm{y}$ direction are used.

Because all simulations differ in placement of wells, placement of fractures and chosen coarsening ratios for fractures and matrix it is not possible to determine which of the two configurations result into the best result. The accuracy of an ADM simulation heavily depends on the position of vertices of fractures and matrix. The reason behind this is that the placement of vertices are the first step to compute basis functions. The computation of basis functions is discussed in the next subsection in which it will be clear how the placement of vertices influence these computations.

\subsubsection{Flexible Coarsening for Fractures}

A coarse grid can also be projected on a fracture. A fracture however, is represented as a 1-D line. This means that it doesn't have faces, only vertex nodes and edges. The coarsening ratio for fractures can be flexibly chosen and can differ per fracture and do not have to be equal to the coarsening ratio given to the matrix. An example of this is visualized in figure 4.3.

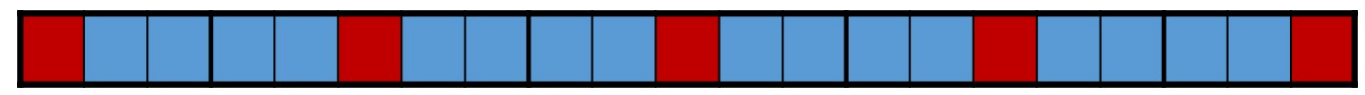

Figure 4.3: A 1-D representation of a coarse grid. This is implemented for fractures when a 2-D matrix is considered.

Fractures and matrix can also coexist in a single domain (see 4.4). In this work the importance of vertices given to fractures is investigated. The flexibility of choosing coarsening ratios per fracture allows the user to choose which fracture is given more vertices. This work aims to research the difference of fractures and how this difference can influence the amount of vertices needed to capture a fracture's effect on flow in a reservoir.

\subsubsection{Computation of Basis Functions}

A basis function is calculated per vertex of a dual coarse grid. This means that for the matrix 4 basis functions are calculated per dual coarse grid. The basis functions are calculated algebraically according to the method described by [36]. Local basis functions are calculated for each coarse 


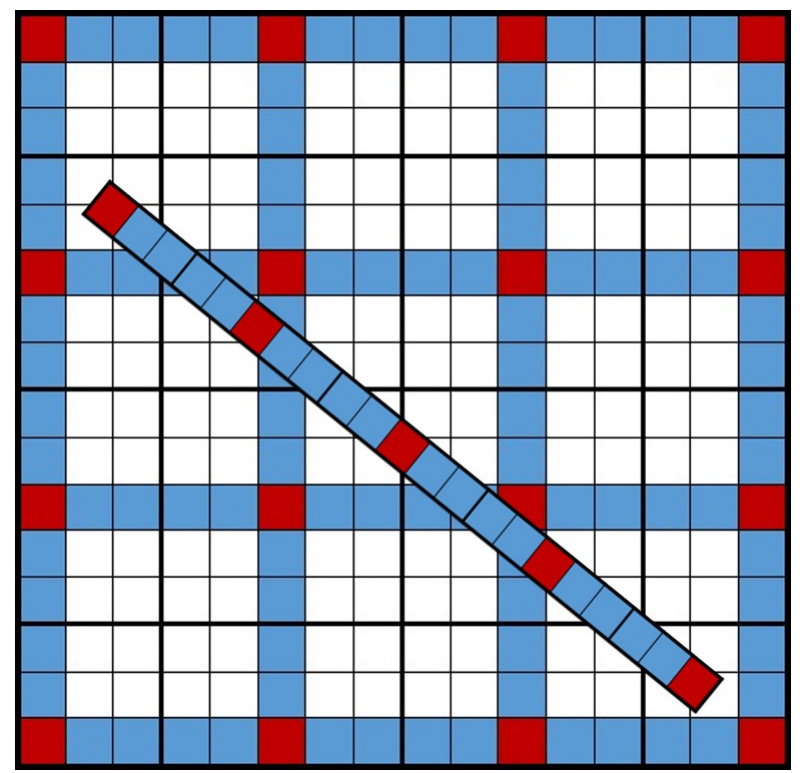

Figure 4.4: A single fracture and matrix coexist in this 2-D domain.

node $i$, corresponding to each dual coarse grid. As described, the pressure in the vertices is set to:

$$
\delta_{i j}= \begin{cases}1, & \text { if } i=j \\ 0, & \text { if } i \neq j\end{cases}
$$

This can be visualized as done in figure 4.5.

In order, the edges (a 1-D line, grids shown in blue in figure 4.5) are resolved, followed by the faces inside the coarse grid block (the 2-D plane represented by white grids in figure 4.5).

It is important to note that using the configuration of 4.3.3, all cells bounded by the vertices set to zero will be non zero. All cells outside this will be zero due to the boundary of zeroes.

In the research performed by Tene [36], the fractures and matrix are fully coupled. This research uses this fully coupled approach, meaning when a fracture is present in the dual coarse grid, this fracture influences the basis functions calculated. How a fracture with a permeability lower than the matrix influences the basis function calculated can be seen in figure 4.6. This figure shows how the fracture cuts off the high values of the basis function to the bottom right corner of the dual coarse grid. The solution for the dual coarse grid behaves as if fluid flow would behave if it were to be blocked by a low permeable fracture.

\subsubsection{Vertices on Fractures}

As can be seen in figure 4.3, fractures have vertices too. A basis function belongs to each vertex. The calculations of the basis functions for a vertex on a fracture can be visualized as done in figure 4.7 .

A dual coarse grid can be seen as an information map of how that particular area of the reservoir behaves with respect to pressure and saturation. In other words, the goal of calculating basis functions is to obtain a local solution. When a fracture exists within a dual coarse grid, as can be seen in 4.7 , some vertices belonging to a fracture may lay outside the dual coarse grid. The fracture visible in figure 4.7 has its next vertex located outside the coarse grid. This 'breaks' the enclosed dual coarse grid's value of zeroes which are located at the boundary of each grid. When 

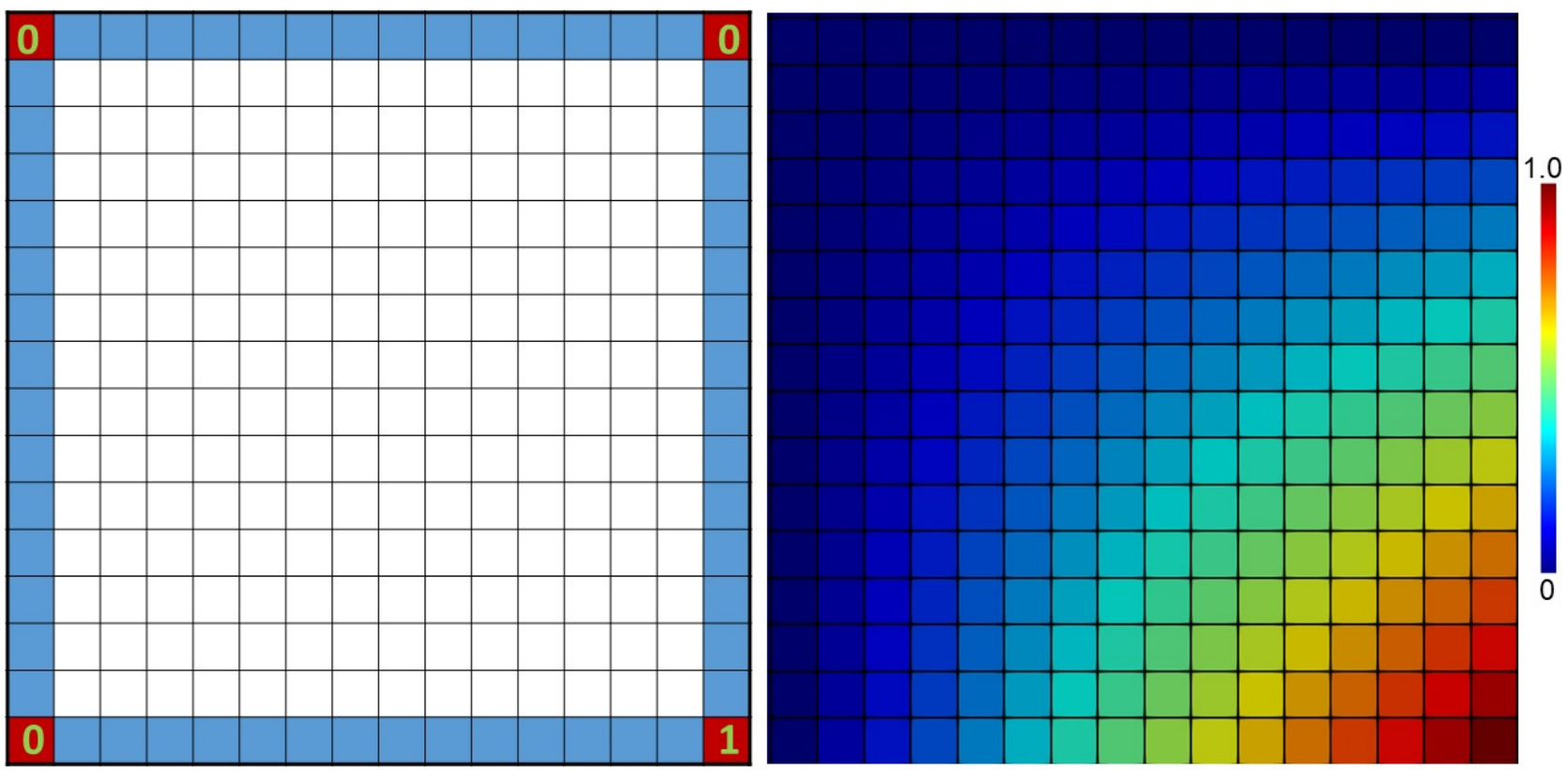

Figure 4.5: Left, a schematic representation of how the vertices are set to calculate the basis function for coarse node $i$. Right, the result of these calculations. All values in this dual coarse grid are between 1 and 0 as can be seen on the scale bar on the far right.
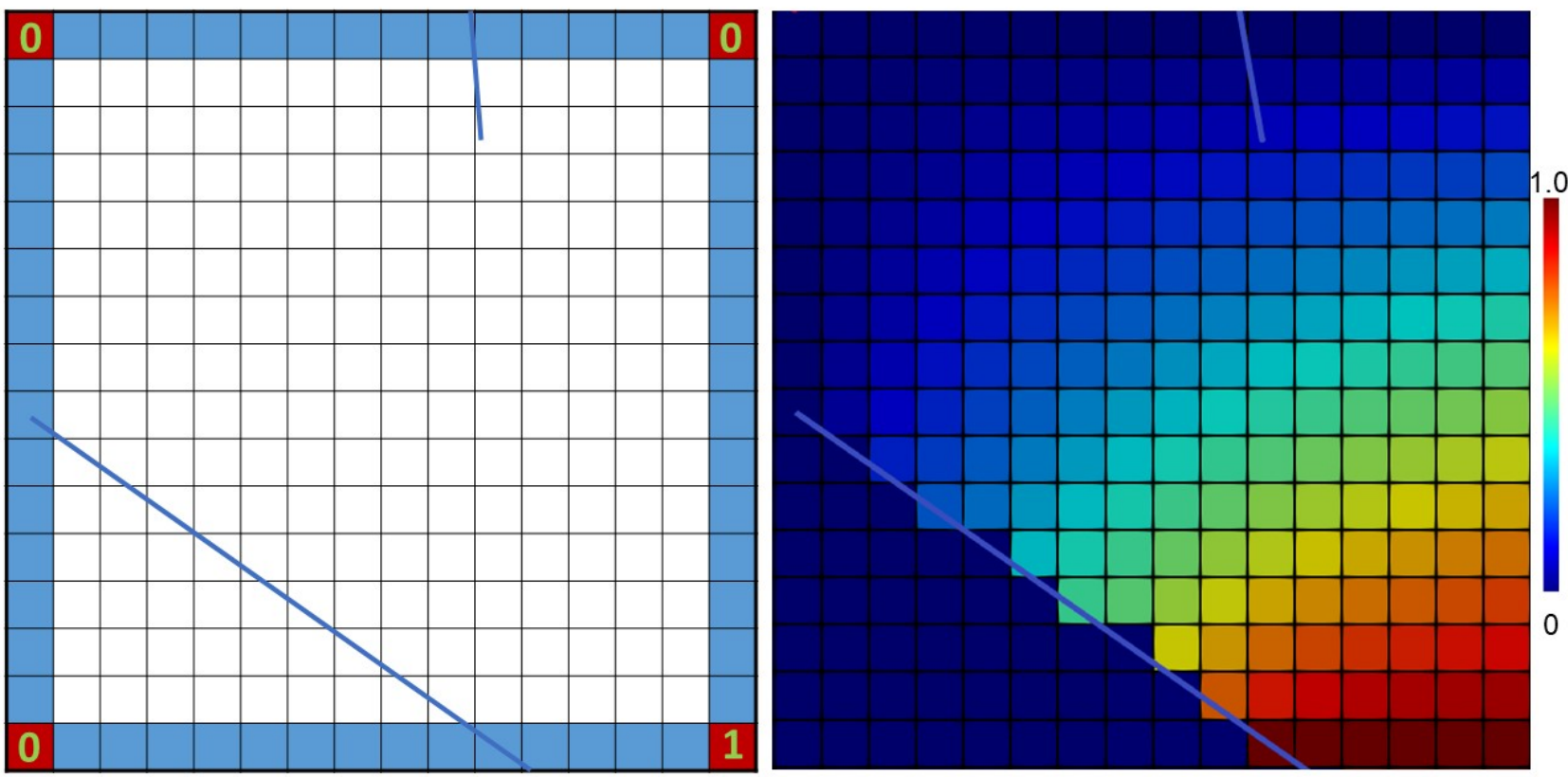

Figure 4.6: Left, a schematic representation of how the vertices are set to calculate the basis function for coarse node $i$ in a dual coarse node containing two fractures. Right, the result of these calculations. Left of the lower fracture all grid cells have value zero as the low permeability of the fracture causes these cells to be cut off from the cells to the right of the fracture. 

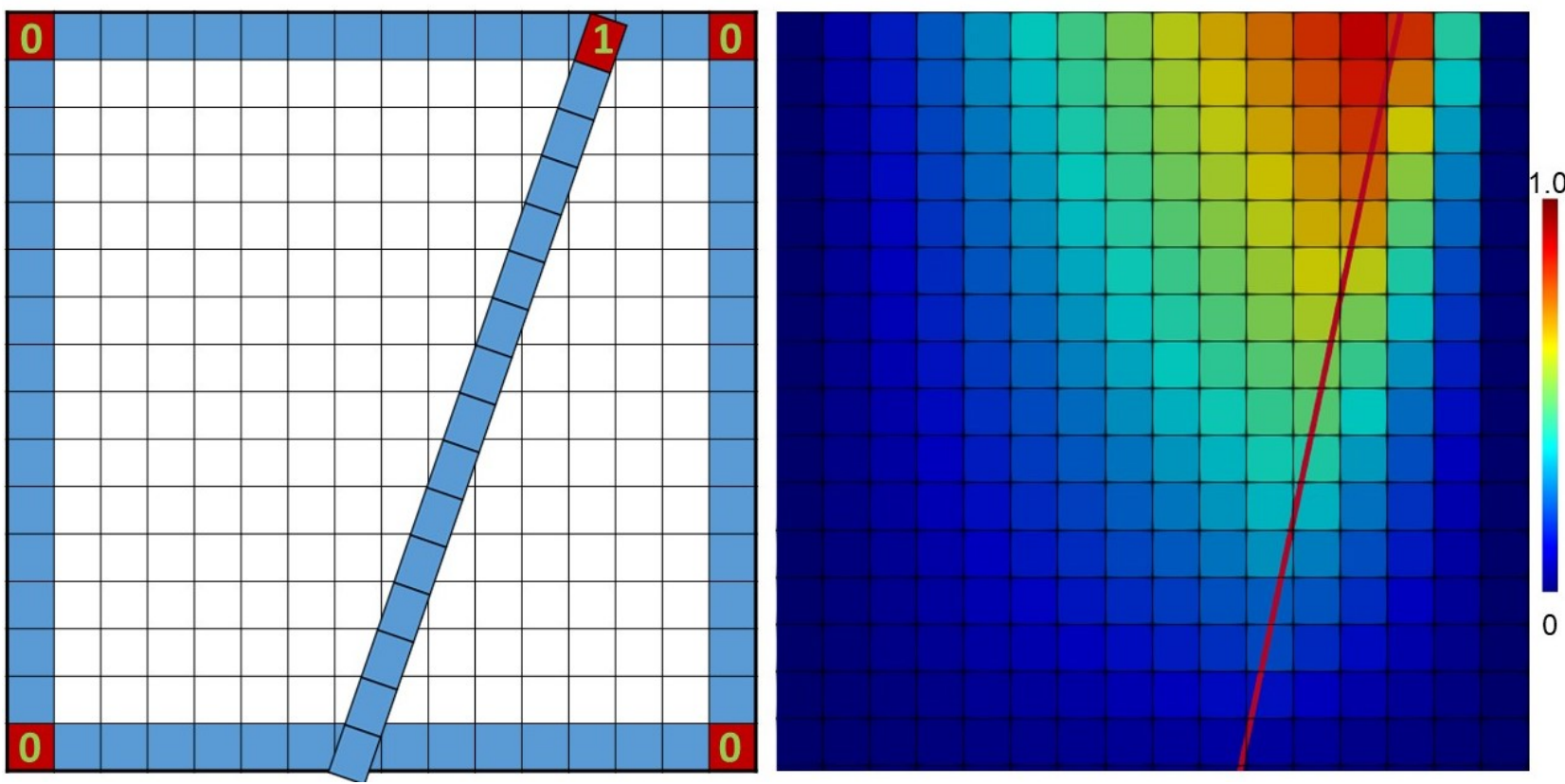

Figure 4.7: Left, a schematic representation of how the vertices are set to calculate the basis function for coarse node $i$ in an dual coarse node containing one fracture. Right, the result of these calculations.

the boundaries are not closed off due to the presence of fractures, the information 'leaks' outside the dual coarse grid and this leads to computationally more expensive basis functions. As the basis functions are calculated at the start of each simulation, these computationally costs can be seen as overhead computational costs.

\subsection{Reducing Computational Cost by Using Multilevel}

This section elaborates on how using the multilevel approach reduces the computational costs. This is shown using the mathematical notation how the simulation is solved. To start, the finescale mathematical notation will be discussed to be followed by the multilevel mathematical notation. At the end of this section, they will be compared.

The notation of finescale simulations is quite simple. It consists of three matrices: the $A$ matrix which is filled with transmissibilities as described in 3.2, the unknown vector pressure, $p$, and the known vector flow, $q$. This can be rewritten as done in equation 4.4.1 to solve for $p_{f}$.

$$
\underbrace{A_{f}}_{N_{f} \times N_{f}} \times \underbrace{p_{f}}_{N_{f} \times 1}=\underbrace{q_{f}}_{N_{f} \times 1} \rightarrow p_{f}=A_{f}^{-1} \times q_{f}
$$

Where $f$ denotes finescale resolution. The sizes of the matrices are given below each matrix. $N_{f}$ stands for the number of finescale grid cells.

To calculate the coarse scale pressure, $p_{c}$ a more complex notation is used.

To create an $A$ matrix for a coarse grid, $A_{c}$, the finescale $A$ matrix, $A_{f}$ has to be multiplied by the 
restiction and prolongation operator, $\mathbf{R}$ and $\mathbf{P}$ respectively. In equation 4.4.2 this is shown.

$$
\underbrace{\boldsymbol{R}}_{N_{c} \times N_{f}} \times \underbrace{A_{f}}_{N_{f} \times N_{f}} \times \underbrace{\boldsymbol{P}}_{N_{f} \times N_{c}}=\underbrace{A_{c}}_{N_{c} \times N_{c}}
$$

Where, $N_{c}$ denotes the number of coarse grid cells. To be able to solve for coarse scale pressure the $q_{f}$ term also needs to be rewritten to a $q_{c}$ term. This is done as in equation 4.4.3.

$$
\underbrace{\boldsymbol{R}}_{N_{c} \times N_{f}} \times \underbrace{q_{f}}_{N_{f} \times 1}=\underbrace{q_{c}}_{N_{c} \times 1}
$$

Using $A_{c}$ and $q_{c}$, the coarse scale pressure, $p_{c}$, can be solved. By rewriting equations 4.4 .2 and 4.4 .3 an approximation of $p_{f}$ can be obtained.

$$
\underbrace{p_{c}}_{N_{c} \times 1}=\underbrace{A_{c}^{-1}}_{N_{c} \times N_{c}} \times \underbrace{q_{c}}_{N_{c} \times 1}=\underbrace{\left(\boldsymbol{R} A_{f} \boldsymbol{P}\right)^{-1}}_{N_{c} \times N_{c}} \times \underbrace{\boldsymbol{R} q_{f}}_{N_{c} \times 1}
$$

To project the coarse scale solution, $p_{c}$, to the finescale grid, the $p_{c}$ vector has to be multiplied by the prolongation operator, $\boldsymbol{P}$. This results in an approximation of the finescale solution, $p_{f}$

$$
\underbrace{p_{f}}_{N_{f} \times 1} \approx \underbrace{\boldsymbol{P}}_{N_{f} \times N_{c}} \times \underbrace{p_{c}}_{N_{c} \times 1}
$$

Using equations 4.4.4 and 4.4.1 an example can be given on how computational costs can be reduced. In the following example a finescale grid of 81 by 81 grids $\left(N_{f}\right)$ is chosen. A coarsening ratio of 3 is imposed with only 1 level of coarsening, leaving 27 by 27 coarse grids cells $\left(N_{c}\right)$. Equation 4.4.1 calculates the finescale solution, $p_{f}$ and equation 4.4.7 the coarse scale solution, $p_{c}$.

$$
\begin{aligned}
& \underbrace{A_{f}^{-1}}_{81 \times 81} \times \underbrace{q_{f}}_{81 \times 1}=\underbrace{p_{f}}_{81 \times 1} \\
& \underbrace{\boldsymbol{P}}_{81 \times 27} \times \underbrace{\left(\boldsymbol{R} A_{f} \boldsymbol{P}\right)^{-1}}_{27 \times 27} \times \underbrace{\boldsymbol{R} q_{f}}_{27 \times 1} \approx \underbrace{p_{f}}_{81 \times 1}
\end{aligned}
$$

When equations 4.4.6 and 4.4.7 are compared it can be seen that in equation 4.4 .6 the 81 by $81 A^{f}$ matrix needs to be solved every timestep, whereas in equation 4.4.7 the biggest matrix that needs to be solved is 27 by 27 . The bigger the finescale grid and the more levels used, the bigger the advantage of multiscale will be.

The $\boldsymbol{P}$ and $\boldsymbol{R}$ matrices are calculated at the beginning of the simulation and are known for the rest of the simulation. Therefor they are only calculated once and are not considered as a high computational cost.

From the example given in this section, it becomes clear how important it can be to minimize the amount of coarse grids cells. Representing fractures with a high amount of coarse grid cells is an easy way to stay accurate, however this work has as a target to find a ratio between accuracy and computational costs. 


\subsection{Algebraic Dynamic Multiscale (ADM)}

In this section, the Algebraic Dynamic Multiscale (ADM) approach is introduced. The MMs and ADM approaches are very similar to each other and all principles for MMs are valid for the ADM approach. However, the ADM approach has an interesting feature which enables the approach to capture sharp saturation fronts. This is because the ADM approach can dynamically switch between grid resolution where the MMs is a static approach with a single resolution only.

In the simulations a combination of finescale grids and higher level grids are used. To capture sharp fronts and areas around wells a front detection method needs to be used. The front is detected and captured as described in this section. Because of the ability of ADM to capture saturation fronts, this work uses it to simulate multiphase simulations. This results in a time dependent simulation according to equation 3.2.2.

Let $\Omega_{l}^{I}$ and $\Omega_{l}^{J}$ be two neighbouring coarse grid cells $I$ and $J$ at coarsening level $l$. The finescale grids belong to coarse grid cells $I$ and $J$ are indicated by $i$ and $j$ respectively. The saturation difference between coarse grid cell $I$ and $J, \Delta S_{I J}$, is what is the deciding factor to refine coarse cell $I$ from level $l$ to level $l-1$. This is calculated as follows:

$$
\Delta S_{I J}=\max \left(\left|S_{i}-S_{j}\right|\right)
$$

Equation 4.5.1 calculates the highest difference between the saturation of a finescale cell in coarse grid $I$ and the saturation of a finescale cell in coarse grid $J$. Once the maximum difference in saturation is calculated this difference is compared to a threshold that is set by the user. The coarse grid cells will be refined from level $l$ to level $l-1$ if the following condition is met:

$$
\Delta S_{I N}>\text { tol }
$$

The condition in 4.5.2 says that if the saturation difference between $I$ and one of its neighbors is greater than the preferred tolerance, the coarse grid cells will be refined from level $l$ to level $l-1$. In a 2D-domain $I$ will have four neighbors, whereas in a 3D-domain $I$ will have six neighbors. This research also looks at how the accuracy of a simulation is effected if fracture cells are also taken into account when the grid refinement is performed. This means that the fracture cells are coupled to the matrix, and thus can be considered as a neighboring cell, when $\Delta S_{I N}$ is compared to the user defined tolerance.

Around the wells a finescale grid is set to capture the source term fluxes accurately. 


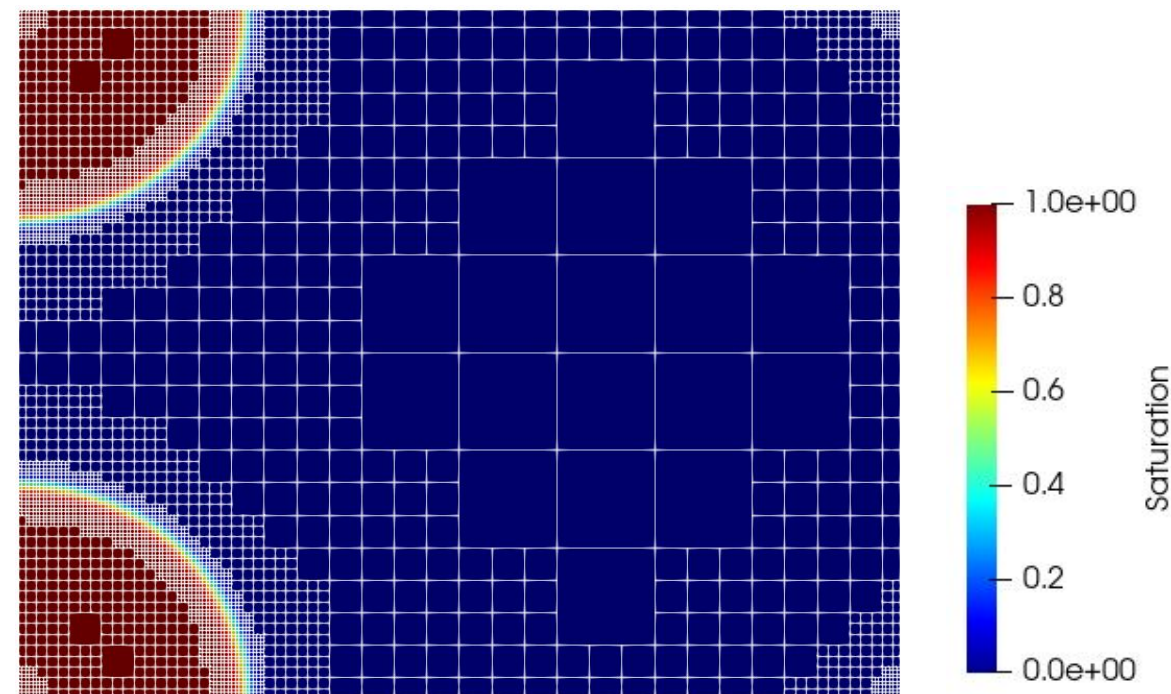

Figure 4.8: This figure shows how different levels are used in a simulation. Notice how the front of the injected phase is surrounded by a finescale resolution and the coarser grid cells are around an area where no gradient in saturation is present. Around the wells (in every corner) a finescale grid is imposed.

It is also important to note that the transition between grid resolution has a maximum of 1 level among the neighboring cells. Grid cells around a cell at level $l$ can be either at level $l-1, l$ or $l+1$. 


\section{Chapter 5}

\section{Static Multilevel Multiscale Results}

In this chapter the results for static Multilevel Multiscale simulation, MMs, approach is presented. As mentioned in chapter 4 the MMs approach is used for single phase simulations in which incompressible fluids are assumed and gravity is not taken into account. For each section the MMs results are compared to a finescale solution for pressure. The error for the MMs and ADM results (chapter 6) is calculated using the following equation:

$$
e_{x}=\frac{\left\|x_{F S}-x_{A D M}\right\|}{\left\|x_{F S}\right\|}
$$

Here, the error of variable $x$ (i.e. saturation or pressure) is denoted as $e_{x}$. The following sections will focus on the flexibility of coarsening ratios implemented to simulate the presence of fractures.

Not only will the error corresponding to the different test cases be presented in this chapter, the MMs solutions will also be compared to the finescale in a visual manner. A trajectory is chosen over the matrix for each test case and the belonging pressure drop to this specific trajectory is plotted for all relevant test cases. From this plot the difference between the finescale solution and the MMs solutions can be visualized.

In this chapter multiple test cases are presented. The first test case is used in multiple papers written on the MMs subject. In this testcase two fractures are present forming a cross in the middel of the matrix. As described in chapter 2, different values of principle stress and different directions of principle stress lead to different permeabilities for fractures. Using different settings for principle stress, the fractures are either both blocking or the vertical fracture is conductive and the horizontal fracture is blocking (and vice versa). The settings used for principle stresses and direction can be found in appendix B. Using the method described in chapter 2 it is not possible to have fractures with a 90 degree different orientation to be both conductive so this has to be set manually.

The next two test cases can be found in literature in a paper written by Bernd Flemisch et al [37]. To represent fractures as in the paper, the fractures are manually set to be conductive or blocking with respect to their permeability. 


\subsection{Simulator settings}

In this section the settings for the simulations run in this chapter will be given.

Table 5.1: Reservoir properties that stay constant throughout all simulations presented in this chapter.

\begin{tabular}{|l|l|l|}
\hline Reservoir Property & Value & Unit \\
\hline Matrix Porosity & 0.2 & - \\
\hline Fluid Density Phase 1 & 1000 & $\mathrm{~kg} / \mathrm{m}^{3}$ \\
\hline Viscosity Phase 1 & $3 \mathrm{e}-3$ & $\mathrm{~Pa} \mathrm{sec}$ \\
\hline Compressibility Phase 1 & 0 & $1 / \mathrm{Pa}$ \\
\hline Reservoir Permeability & $1 \mathrm{e}-14$ & $\mathrm{~m}^{2}$ \\
\hline Injection Well Pressure & $5 \mathrm{e} 7$ & $\mathrm{~Pa}$ \\
\hline Production Well Pressure & $1 \mathrm{e} 7$ & $\mathrm{~Pa}$ \\
\hline Fracture Aperture & $5 \mathrm{e}-3$ & $\mathrm{~m}$ \\
\hline
\end{tabular}

Besides these simulator settings, all fractures are manually given 64 finescale gridcells. This is imposed to be able to know exactly how many coarse scale grid nodes the fractures are assigned. Using equation 4.3.1 this is calculated.

In the presented test cases four MMs simulations are compared to the finescale solution. In all MMs simulations the matrix has an imposed coarse grid with a coarsening ratio of 3 for the $\mathrm{x}$ and $\mathrm{y}$ direction up to one level. This remains the same throughout the four different MMs simulations. The difference between the MMs simulations is the coarsening ratio given to the fractures. Coarsening ratios of 3, 9 and 63 are imposed on the fractures. Using these coarsening ratios the amount of coarse scale grid nodes is 22,8 and 2 . If coarse scale grid nodes are placed on the fracture, two is the minimum amount of coarse scale grid nodes that can be placed on a fracture.

The last MMs simulation doesn't impose any coarse nodes on the fractures. This can be seen as homogenizing the fractures in the multiscale grid. However, they are not totally homogenized as they are still present when basis functions for the matrix are calculated. The homogenized fracture therefore only has a finescale grid but as all MMs simulations are static and use the highest level of coarsening, the homogenized fracture does not have a grid in the multiscale solution.

\subsection{1. pEDFM settings}

In section 3.5 the pEDFM extension is introduced and discussed. This extension has an influence on transmissibility, T, by multiplying it by $\alpha$. It is described that when $\alpha$ is 1 , the connection between two matrix cells is cut off. This is also the case for the calculation of basis functions.

Relaxing the $\alpha$ factor to be slightly smaller than 1 allows for leakage. This leakage will help the passage of information across the dual coarse grid. Therefor the basis functions can capture the heterogeneity more accurately. In the following test cases the $\alpha$ is reduced by 0.01 to allow for leakage. 


\subsection{Cross Test Case}

In this section the results belonging to the cross test case will be presented. The test case consists of 4 wells of which two are injectors and two are producers. They generate a horizontal flow with two injectors placed on the left corners and the producers placed on the right corners. The cross test case consists of a matrix with 163 by 163 grids in the $\mathrm{x}$ and y direction. This number of gridcells corresponds to a coarse node configuration where they are placed in the corners (see section 4.3.1).

\subsubsection{Fully Permeable Cross}

In this subsection the results for the fully permeable cross are presented. The fracture set up is shown in figure 5.1.
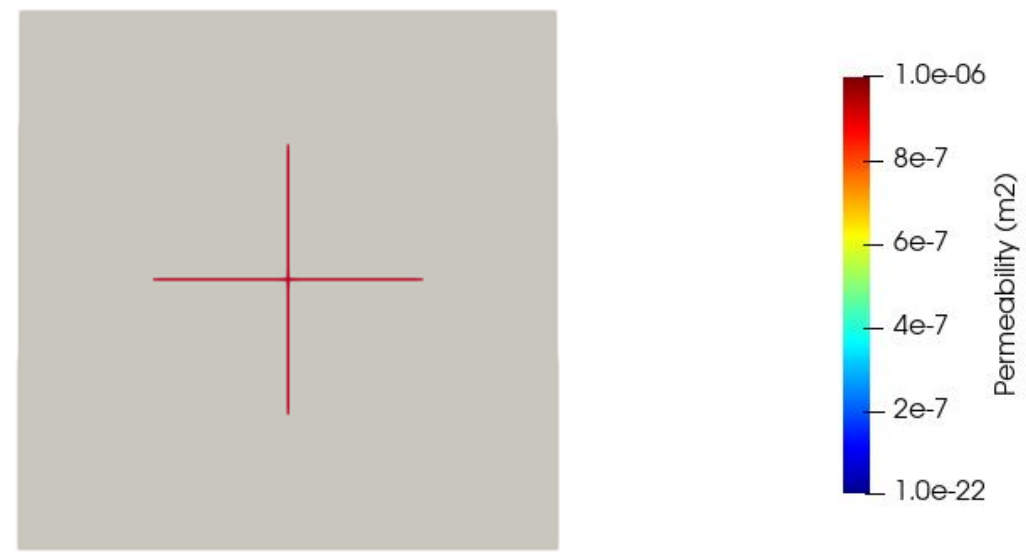

Figure 5.1: The fracture map used for the test cases mentioned below. Fractures with a high permeability are presented in red whereas fractures with a low permeability are presented in blue.

The results for the different simulations for this specific test case are all very similar. At sight all the pressure plots in figure 5.2 look the same. To show the difference between the plots the following figure shows how the solutions between the different coarsening ratios and the finescale solution can be seen.

From the plotted pressure curves in figure 5.3 it is clear that it doesn't matter which coarsening ratio is given to a fracture. In figure 5.3 two curves are clearly visible. This is because the homogenized case overlaps the plot for scenarios CR3, CR9 and CR63. Only when zoomed in very closely, the difference becomes visible for the human eye. To not only visualize the difference in the plots, the error per scenario is given in table 5.2. This is calculated as mentioned in the beginning of this chapter, equation 5.0.1. 


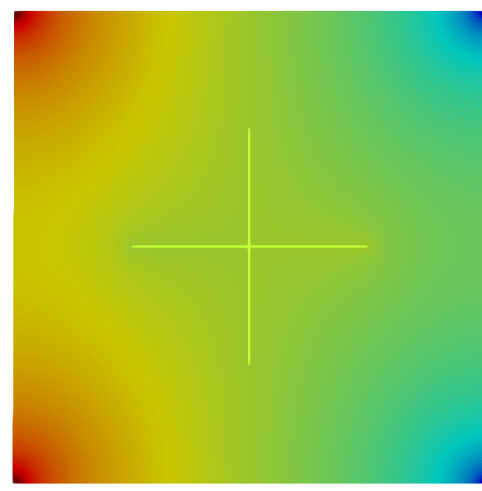

(a) Finescale

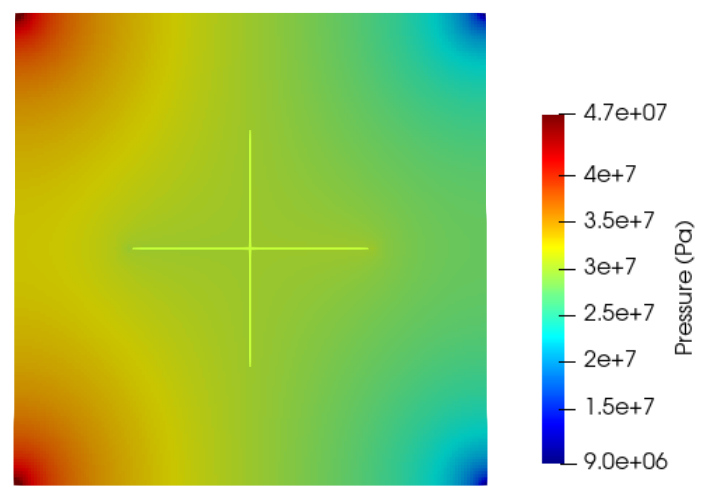

(b) Matrix CR3

Fractures CR3

$\mathrm{e}=0.59 \%$

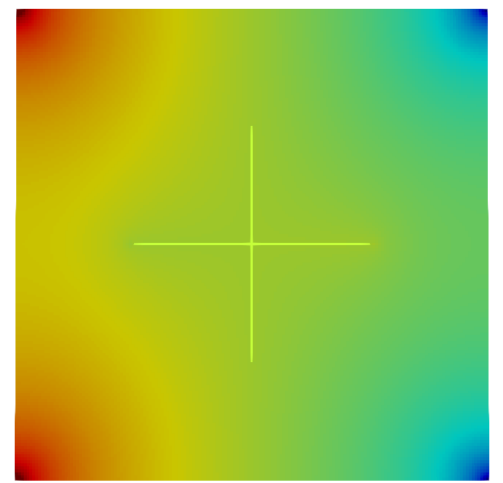

(c) Matrix CR3

Fractures CR9

$\mathrm{e}=0.59 \%$

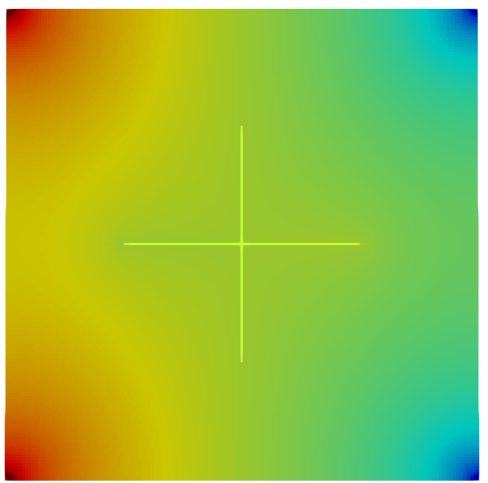

(d) Matrix CR3

Fractures CR63

$\mathrm{e}=0.59 \%$

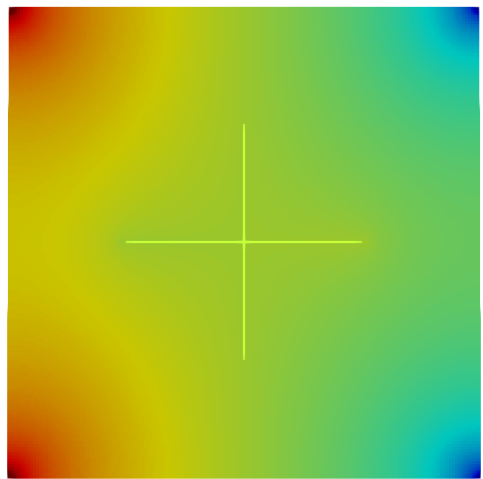

(e) Matrix CR3

Fractures Homogenized

$\mathrm{e}=0.59 \%$

Figure 5.2: The figures a-e are a visualization of the single phase pressure solution. Here, e is the error as computed by equation 5.0.1.

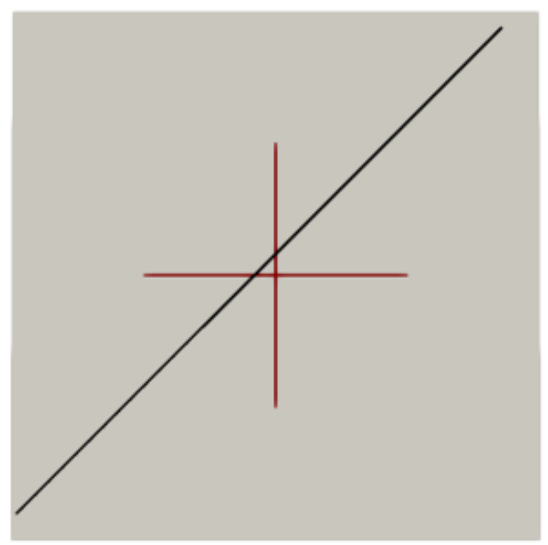

(a)

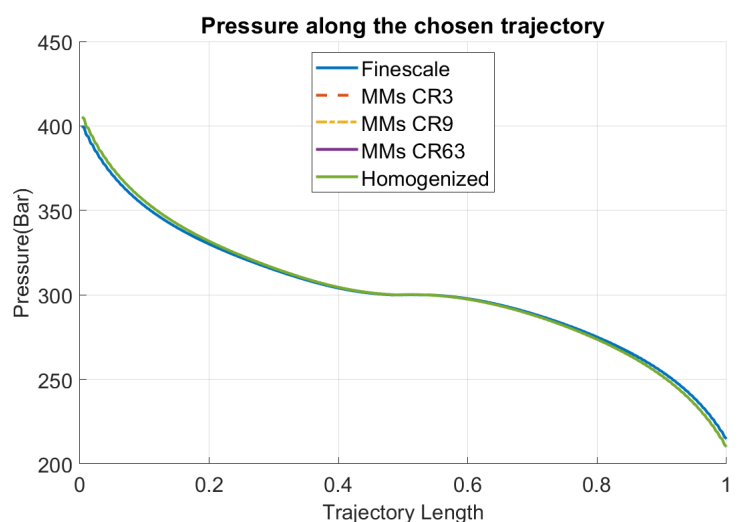

(b)

Figure 5.3: (a) Fracture map with the black line being the trajectory over which the pressure is plotted. (b) The pressure decline belonging to the trajectory shown in (a). The trajectory length is dimensionless. 


\subsubsection{Fully Impermeable Cross}

In this subsection the results for the fully impermeable cross are presented. The fracture set up is shown in figure 5.4 .
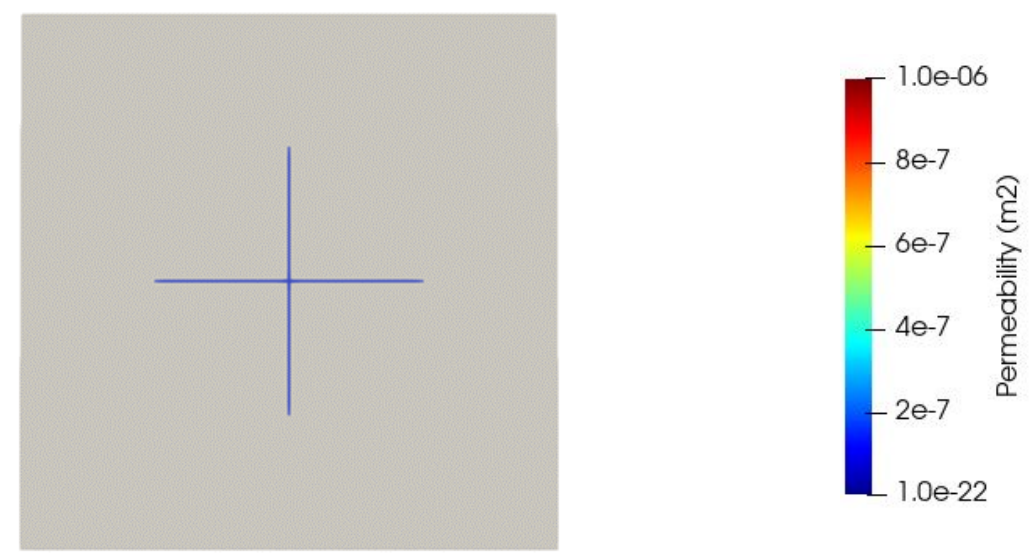

Figure 5.4: The fracture map used for the test cases mentioned below. Fractures with a high permeability are presented in red whereas fractures with a low permeability are presented in blue.

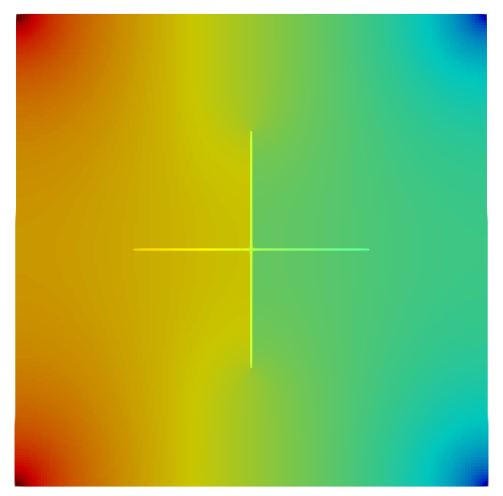

(a) Finescale

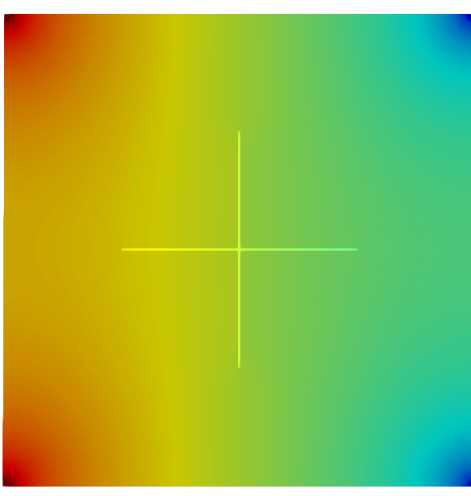

(b) Matrix CR3

Fractures CR3

$\mathrm{e}=2.23 \%$

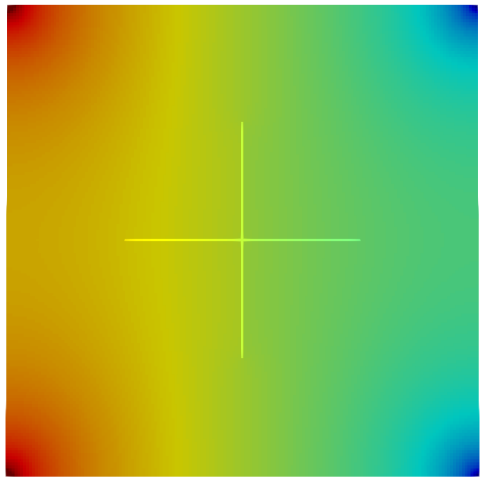

(c) Matrix CR3

Fractures CR9

$\mathrm{e}=2.23 \%$

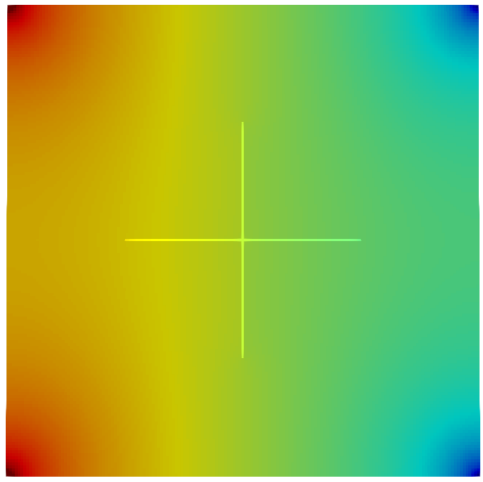

(d) Matrix CR3

Fractures CR63

$\mathrm{e}=2.23 \%$

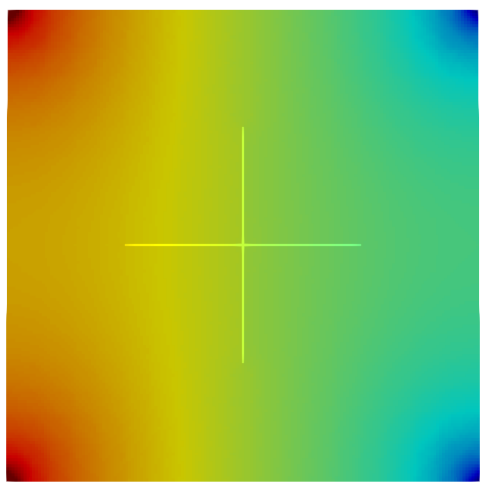

(e) Matrix CR3

Fractures Homogenized

$\mathrm{e}=2.23 \%$

Figure 5.5: The figures a-e are a visualization of the single phase pressure solution. Here, e is the error as computed by equation 5.0.1. 
In figure 5.5 a more visible difference can be seen in the pressure plots for finescale and MMs. The sharp pressure drop after the vertical fracture is less sharp in the MMs solutions. However, between the different coarsening ratios little difference can be seeen. This is also the case in the pressure plot along the chosen trajectory.

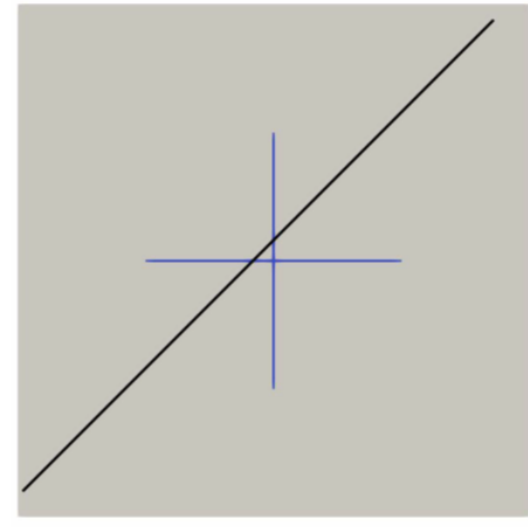

(a)

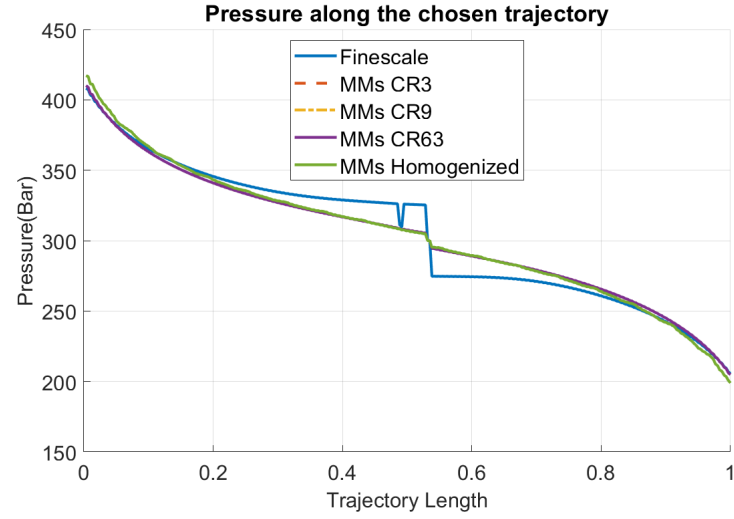

(b)

Figure 5.6: (a) Fracture map with the black line being the trajectory over which the pressure is plotted. (b) The pressure decline belonging to the trajectory shown in (a). The trajectory length is dimensionless.

The pressure plot along the chosen trajectory shows that MMs is not far off from the finescale solution but is not able to capture the pressure drop as sharply as the finescale solution. Between the MMs solutions it is not possible to pick a coarsening ratio which is the most accurate as they all seem to have nearly the same pressure plot. From the overall error shown in table 5.2 this is confirmed as all the coarsening ratios have the same error. 


\subsubsection{Vertical Fracture Permeable, Horizontal Fracture Impermeable}

In this subsection the results for the permeable vertical fracture and impermeable horizontal fracture cross are presented. The fracture set up is shown in figure 5.10.
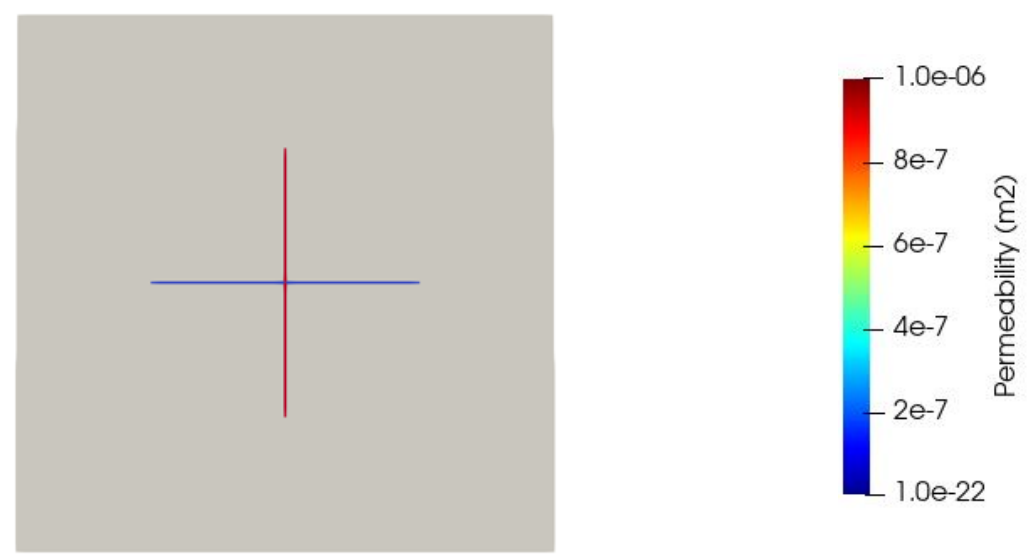

Figure 5.7: The fracture map used for the test cases mentioned below. Fractures with a high permeability are presented in red whereas fractures with a low permeability are presented in blue.

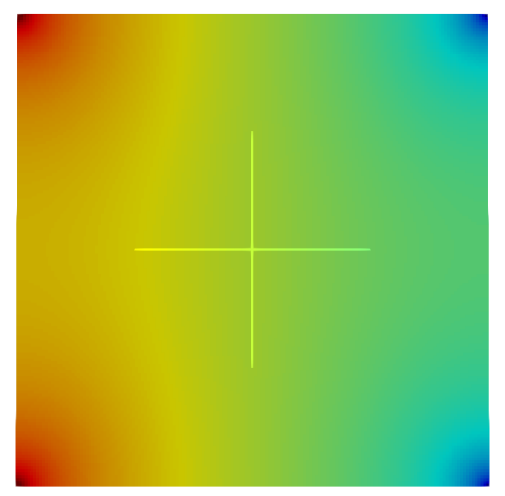

(a) Finescale

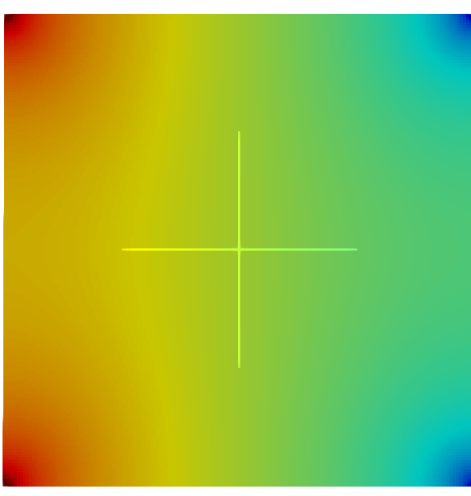

(b) Matrix CR3

Fractures CR3

$\mathrm{e}=0.51 \%$

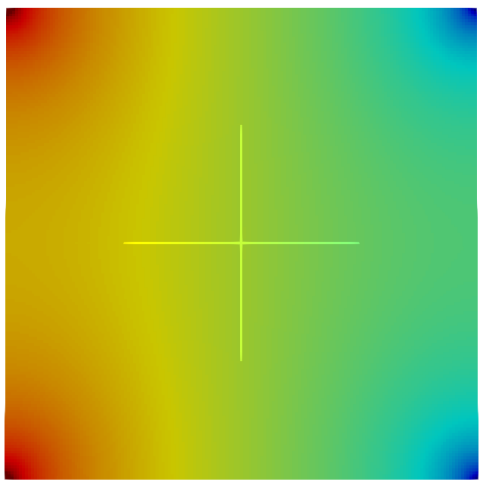

(c) Matrix CR3

Fractures CR9 $\mathrm{e}=0.52 \%$

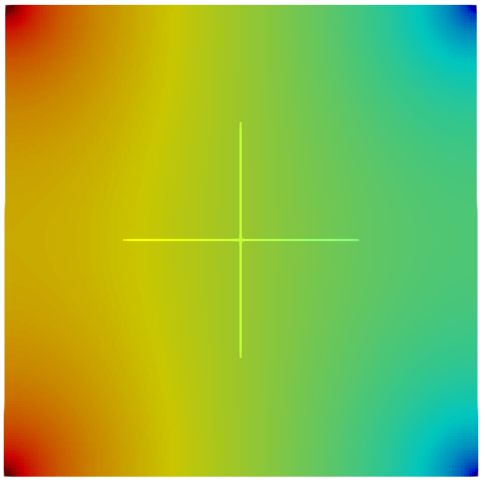

(d) Matrix CR3 Fractures CR63

$$
\mathrm{e}=0.52
$$

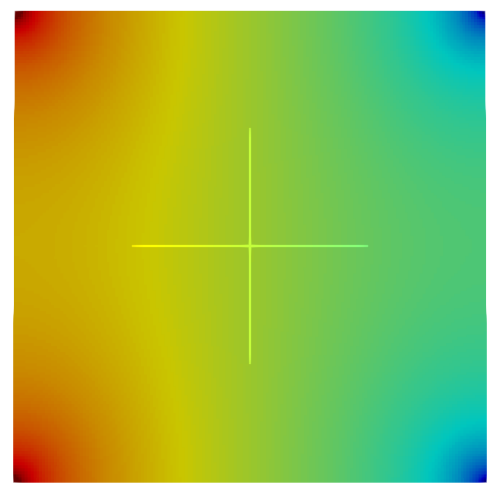

(e) Matrix CR3

Fractures Homogenized $\mathrm{e}=0.53 \% \%$

Figure 5.8: The figures a-e are a visualization of the single phase pressure solution. Here, e is the error as computed by equation 5.0.1. 
Also for the test case in which the two fractures have different permeability values, the difference between the MMs solutions is hard to see. Not only the MMs solutions look like each other, the finescale solution looks very similar to the MMs solutions. This is also visible in the pressure plot along the chosen trajectory. The pressure plot along the chosen trajectory is visible in figure 5.9

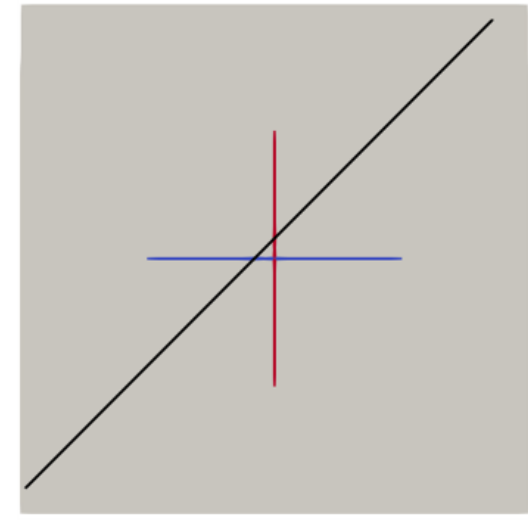

(a)

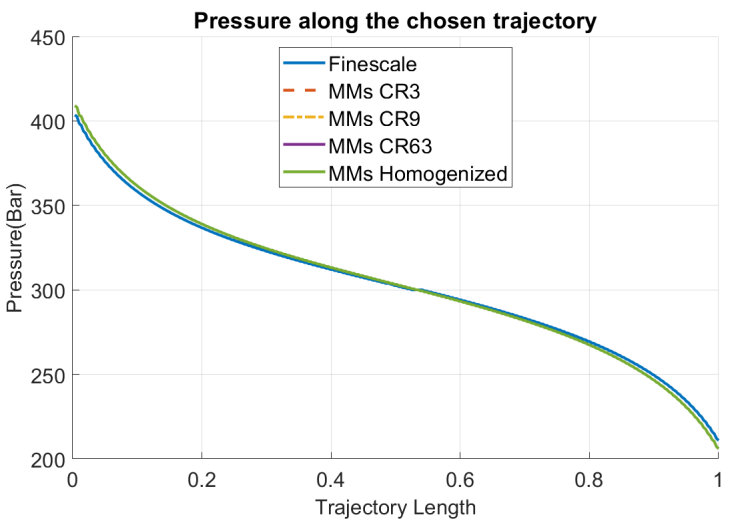

(b)

Figure 5.9: (a) Fracture map with the black line being the trajectory over which the pressure is plotted. (b) The pressure decline belonging to the trajectory shown in (a). The trajectory length is dimensionless.

From the pressure plot in figure 5.9 it can be seen that the MMs solutions are all very close to the finescale solution. Again, all the coarsening ratios give an outcome which differs so little that the human eye cannot see the difference. 


\subsubsection{Horizontal Fracture Permeable, Vertical Fracture Impermeable}

In this subsection the results for the permeable horizontal fracture and impermeable vertical fracture cross are presented. The fracture set up is shown in figure 5.10.
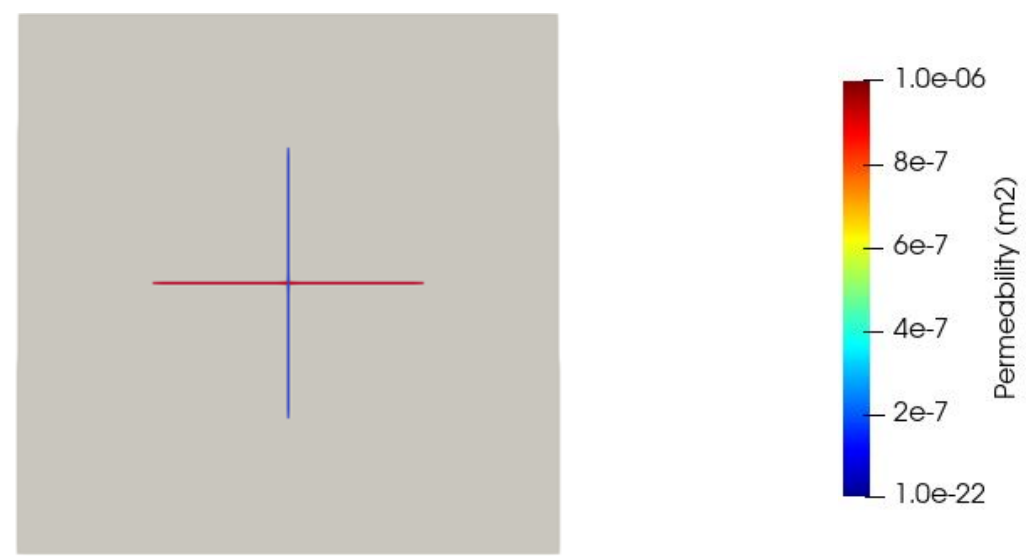

Figure 5.10: The fracture map used for the test cases mentioned below. Fractures with a high permeability are presented in red whereas fractures with a low permeability are presented in blue.

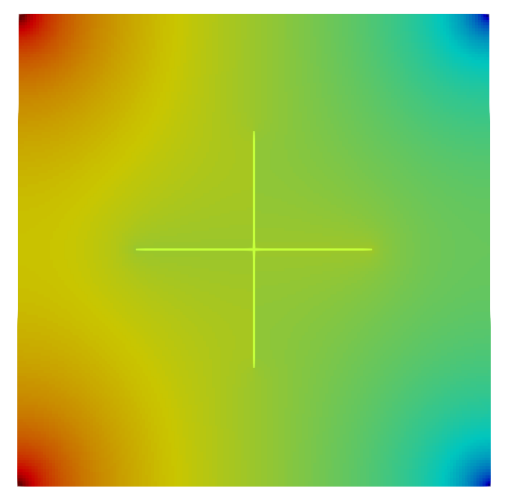

(a) Finescale

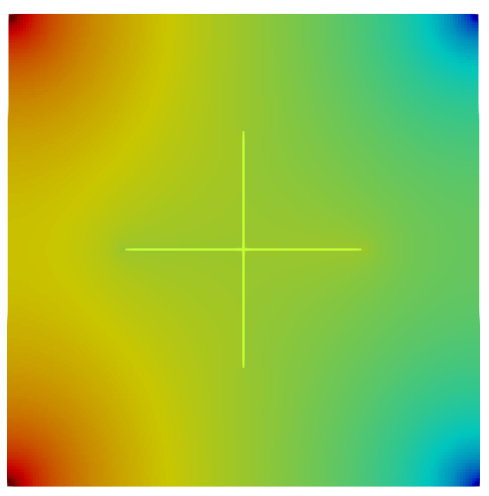

(b) Matrix CR3

Fractures CR3

$$
\mathrm{e}=0.72 \%
$$

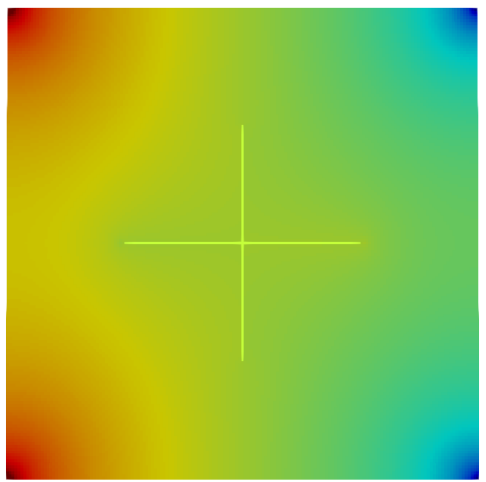

(c) Matrix CR3

Fractures CR9

$$
\mathrm{e}=0.72 \%
$$

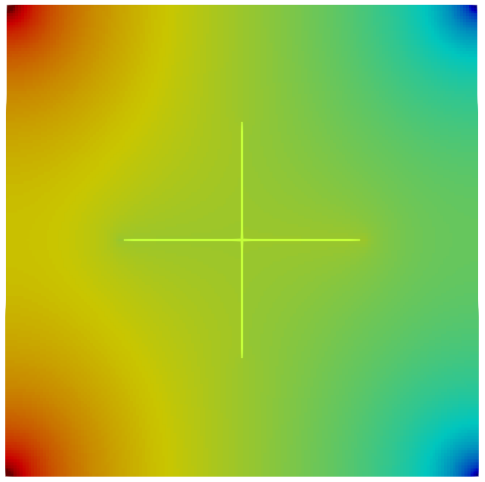

(d) Matrix CR3

Fractures CR63

$$
\mathrm{e}=0.72 \%
$$

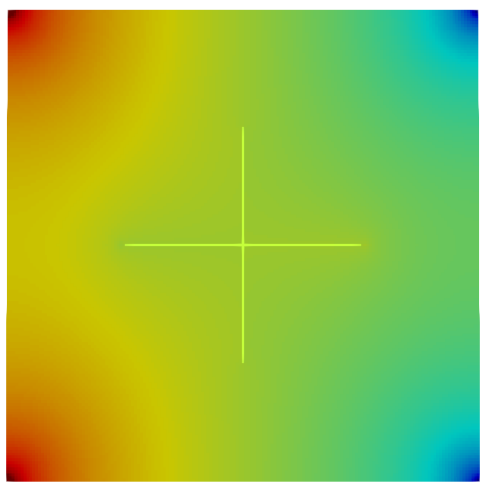

(e) Matrix CR3

Fractures Homogenized

$$
\mathrm{e}=0.72 \%
$$

Figure 5.11: The figures a-e are a visualization of the single phase pressure solution. Here, e is the error as computed by equation 5.0.1. 
In the last test case with the cross fracture configuration, the differences between the finescale solution and MMs solutions is again difficult to see with the naked eye. In the plot showing the pressure along the chosen trajectory the difference becomes more clear.

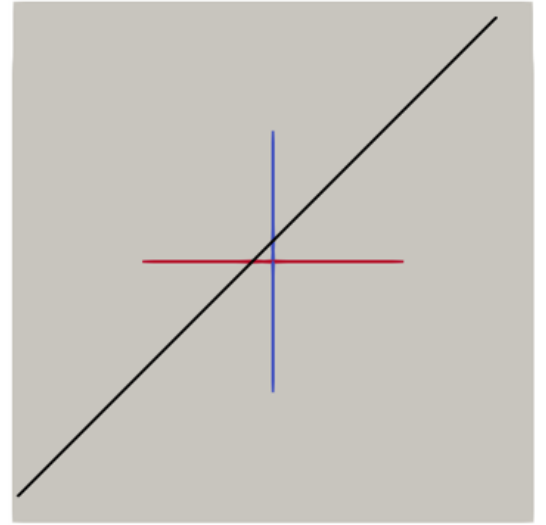

(a)

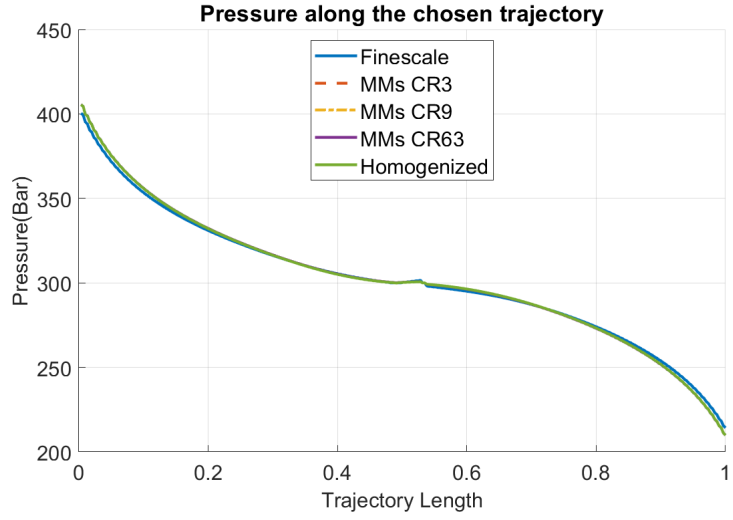

(b)

Figure 5.12: (a) Fracture map with the black line being the trajectory over which the pressure is plotted. (b) The pressure decline belonging to the trajectory shown in (a). The trajectory length is dimensionless.

In figure 5.12 the results show that the MMs solutions are a very good representation of the pressure when compared to the finescale solution. Again, using coarsening ratio 3, 9, 63 or homogenizing fractures in MMs will not improve the accuracy of the simulation.

\subsubsection{Error table}

In table 5.2, the errors are shown for each MMs pressure solution when compared to finescale. The way this is calculated is shown in the beginning of this chapter.

Table 5.2: The table shows the absolute errors for the cross test case.

\begin{tabular}{|l|l|l|l|l|}
\hline Fracture permeability & $\begin{array}{l}\text { Error } \\
\text { CR 3 (\%) }\end{array}$ & $\begin{array}{l}\text { Error } \\
\text { CR 9 (\%) }\end{array}$ & $\begin{array}{l}\text { Error } \\
\text { CR63 (\%) }\end{array}$ & $\begin{array}{l}\text { Error } \\
\text { Homogenized (\%) }\end{array}$ \\
\hline Fully Permeable & 0.59 & 0.59 & 0.59 & 0.59 \\
\hline Fully Impermeable & 2.23 & 2.23 & 2.23 & 2.23 \\
\hline $\begin{array}{l}\text { Vertical Permeable, } \\
\text { Horizontal Impermeable }\end{array}$ & 0.51 & 0.52 & 0.52 & 0.53 \\
\hline $\begin{array}{l}\text { Vertical Impermeable, } \\
\text { Horizontal Permeable }\end{array}$ & 0.72 & 0.72 & 0.72 & 0.72 \\
\hline
\end{tabular}

From the results in table 5.2, it becomes more clear that the coarsening ratio of 63 performs just as well as a coarsening ratio of 3 . Decreasing the amount of coarse scale grid nodes in this case does not influence the accuracy of the pressure solution. The biggest difference between the finescale solution and the MMs testcases can be seen in the fully impermeable cross, followed by the combination of a vertical impermeable and horizontal permeable fracture. 


\subsection{Benchmark 1 Test Case}

In this section the results belonging to the first Benchmark test case will be presented. In this test case pressure throughout a matrix containing 10 fractures is simulated. To simulate the the scenario as presented by Bernd Flemisch et al. [37], eight fractures are highly permeable and 2 have blocking characteristics. The placement of the fractures remains the same however the placement of wells differs in the first two scenarios of this test case. In the first scenario two injection wells are placed in the left corners and two producer wells are placed in the right corners. This will result in horizontal flow. In the next scenario two injector wells will be placed in the top corners and two producer wells will be placed in the bottom corners. This will result into vertical flow.

The matrix consists of 100 by 100 gridcells, placing the multiscale coarse nodes on the corners according to section 4.3.1.

\subsubsection{Horizontal Flow, Benchmark Settings}

In this section the results for the benchmark settings with horizontal flow is presented. The fracture set up is shown in figure 5.13.
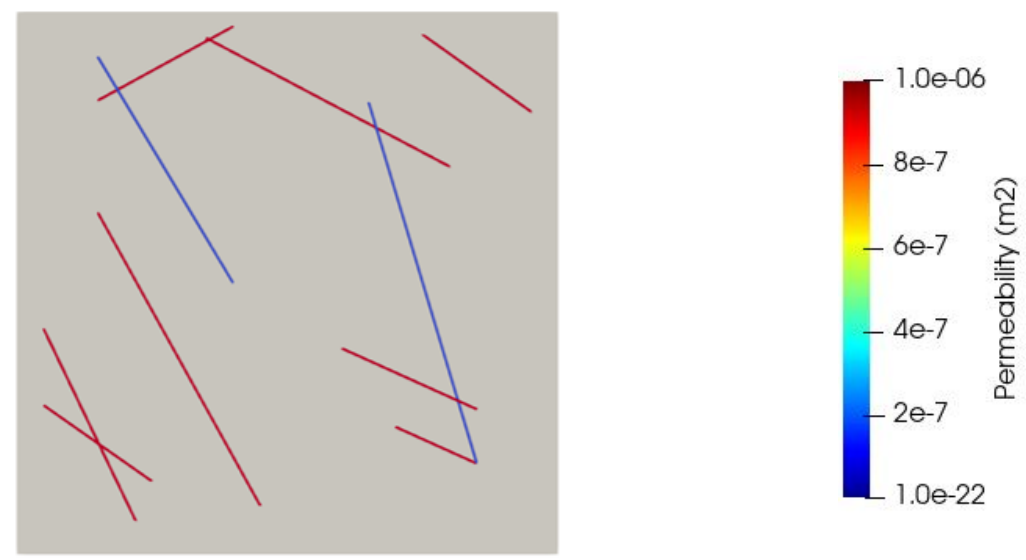

Figure 5.13: The fracture map used for the test cases mentioned below. Fractures with a high permeability are presented in red whereas fractures with a low permeability are presented in blue.

In figure 5.14 the pressure plots can be seen for the different coarsening ratios for fractures in MMs and a pressure plot for finescale. What is very clear to see is that homogenizing fractures shows a very different plot when it's compared to the other MMs solutions and the finescale solution. With fractures not given any coarse nodes, the simulation does not capture the pressure drop accurately. This is not only clear from the plots in figure 5.14, this is also visible in figure 5.15 where the pressure is plotted across the chosen trajectory, shown in black.

From figure 5.15 it can be seen how homogenizing the fractures results into a solution that is inaccurate. In this case the MMs results are comparable to the finescale solution. As can be seen in the testcases with the cross shaped fractures, the coarsening ratio of the fractures doesn't effect the solution. In table 5.3.5 the error across the entire field with respect to the finescale is shown for the horizontal flow and benchmark settings simulation. 


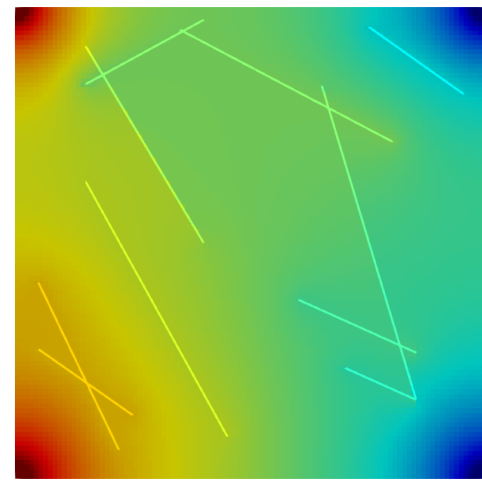

(a) Finescale

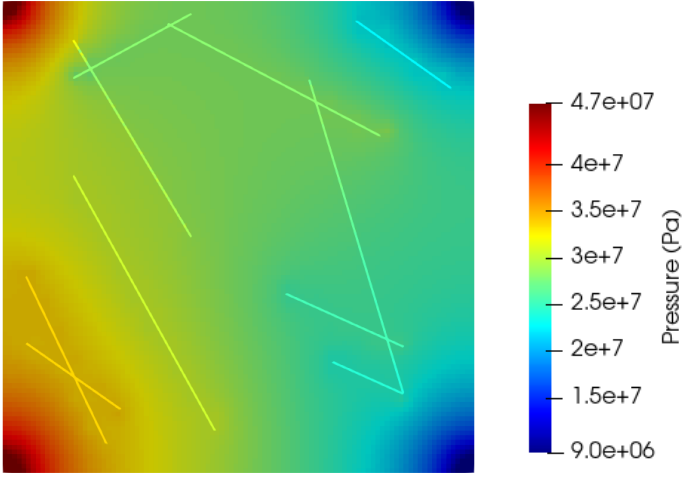

(b) Matrix CR3

Fractures CR3

$\mathrm{e}=0.67 \%$

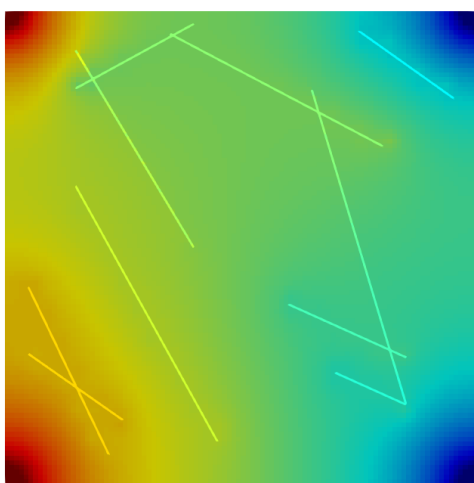

(c) Matrix CR3 Fractures CR9 $\mathrm{e}=0.66 \%$

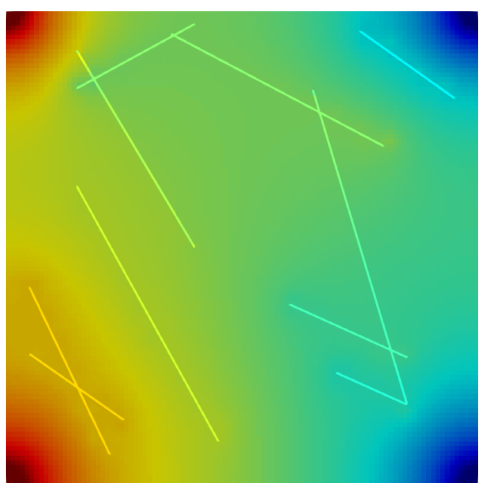

(d) Matrix CR3 Fractures CR63 $\mathrm{e}=0.66 \%$

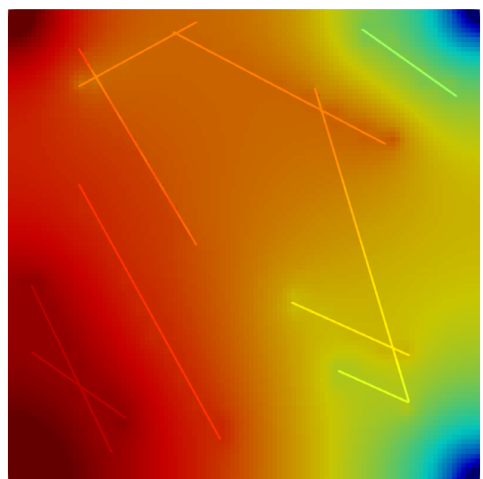

(e) Matrix CR3

Fractures Homogenized $\mathrm{e}=18.29 \%$

Figure 5.14: The figures a-e are a visualization of the single phase pressure solution. Here, e is the error as computed by equation 5.0.1.

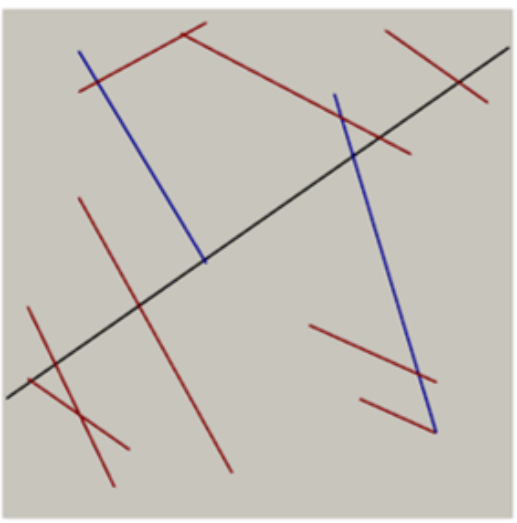

(a)

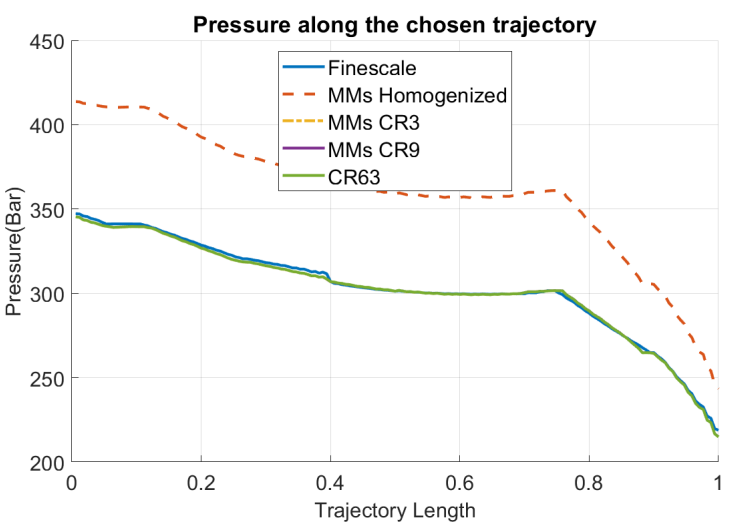

(b)

Figure 5.15: (a) Fracture map with the black line being the trajectory over which the pressure is plotted. (b) The pressure decline belonging to the trajectory shown in (a). The trajectory length is dimensionless. 


\subsubsection{Vertical Flow, Benchmark Settings}

In this section the results for the benchmark settings with vertical flow is presented. The fracture setup is shown in figure 5.13.

In figure 5.16 the pressure plots can be seen for different coarsening ratios for the fractures and a pressure plot for finescale.

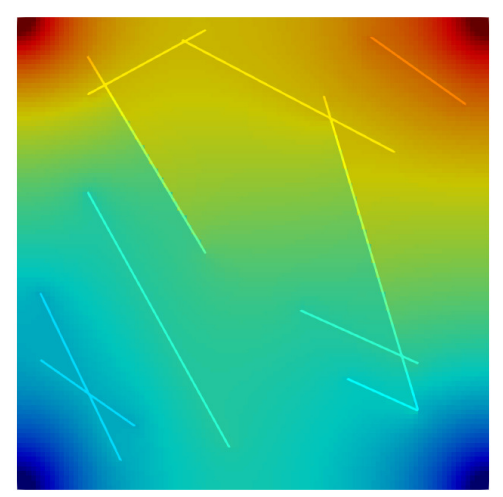

(a) Finescale

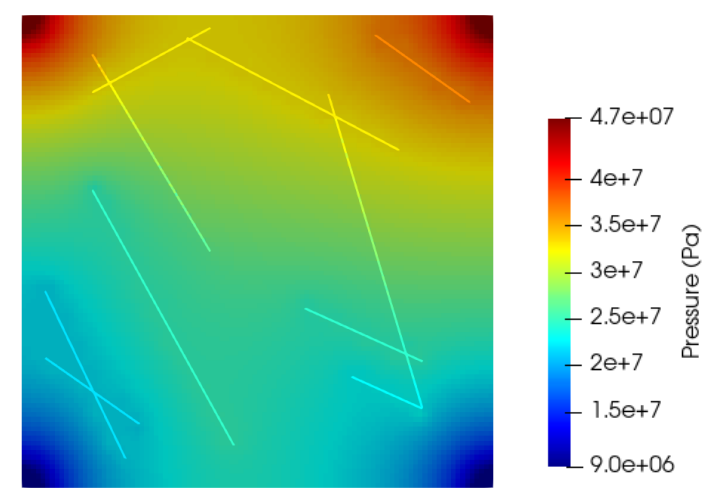

(b) Matrix CR3

Fractures CR3 $\mathrm{e}=0.67 \%$

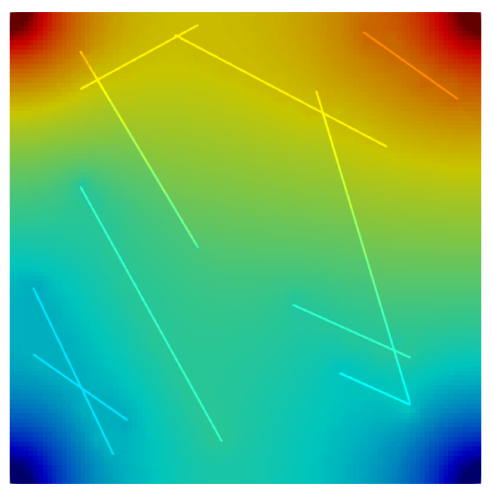

(c) Matrix CR3 Fractures CR9 $\mathrm{e}=0.67 \%$

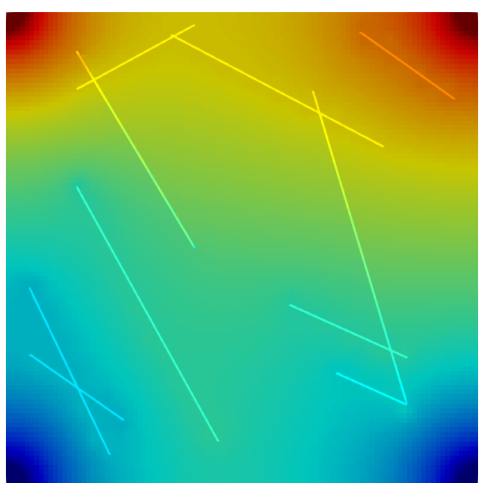

(d) Matrix CR3 Fractures CR63 $\mathrm{e}=0.67 \%$

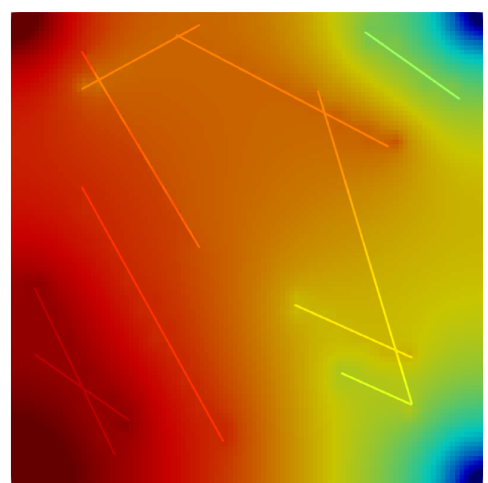

(e) Matrix CR3

Fractures Homogenized $\mathrm{e}=0.67 \%$

Figure 5.16: The figures a-e are a visualization of the single phase pressure solution. Here, e is the error as computed by equation 5.0.1.

Again, it can be seen that the MMs homogenized fracture testcase is not resulting in an accurate solution. The MMs with fractures explicitly represented however, shows how adding coarse nodes to fractures can enable an MMs solution to come close to the finescale solution. In figure 5.17 the pressure is plotted along the chosen trajectory to visualize the difference between each scenario. 


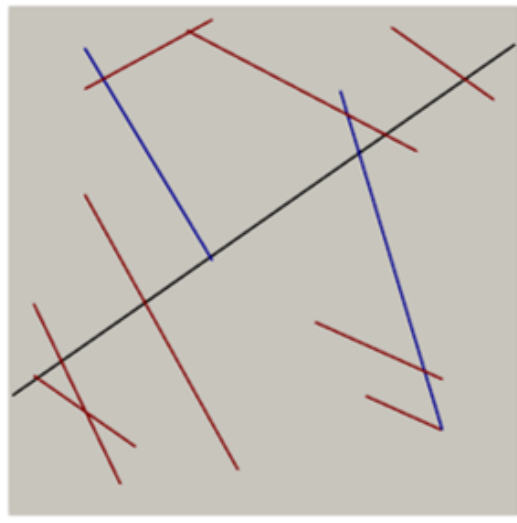

(a)

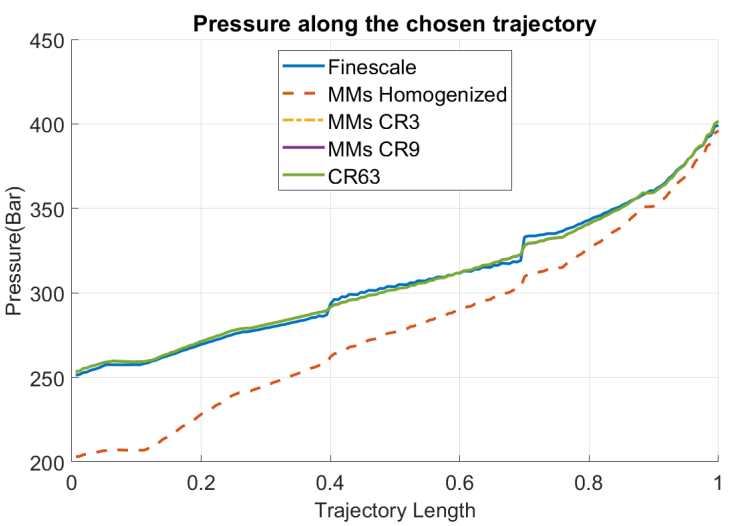

(b)

Figure 5.17: (a) Fracture map with the black line being the trajectory over which the pressure is plotted. (b) The pressure decline belonging to the trajectory shown in (a). The trajectory length is dimensionless.

Also from figure 5.17 it is possible to see the difference between the solution for a finescale simulation, a MMs simulation with fractures explicitly represented and an MMs solution in which no fractures are explicitly represented or homogenized. The difference between the different coarsening ratios is not visible in these plots as it is very small. In table 5.3.5 the error across the entire field with respect to the finescale is shown for the vertical flow and benchmark settings simulation. 


\subsubsection{Permeable Fractures, Horizontal Flow}

In this section the results are presented for a test case in which all fractures are permeable but are placed according to the benchmark test case by Bernd Flemisch et al. [37]. The fracture setup is shown in figure 5.18.
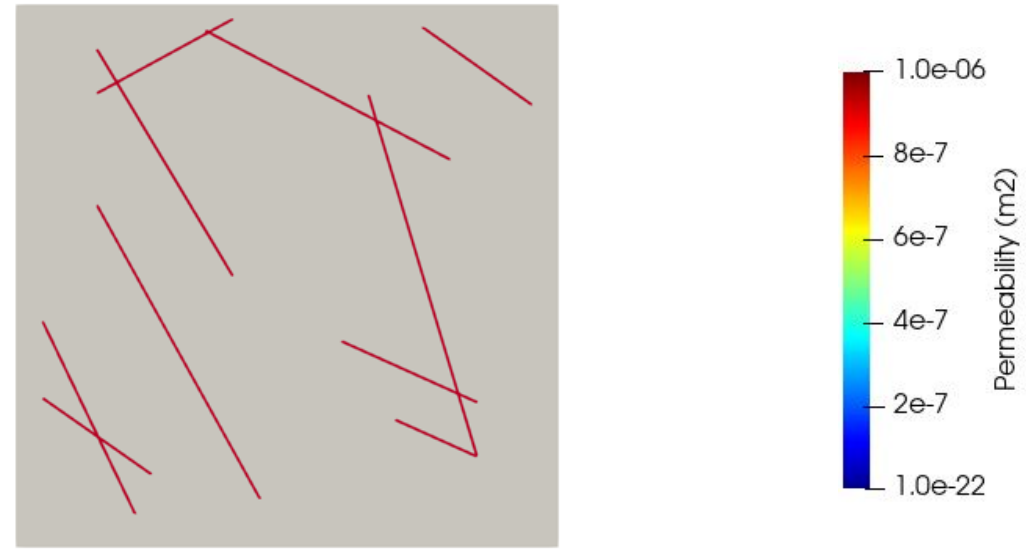

Figure 5.18: The fracture map used for the test cases mentioned below. Fractures with a high permeability are presented in red whereas fractures with a low permeability are presented in blue.

In figure 5.19 the pressure plots can be seen for different coarsening ratios for the fractures and a pressure plot for finescale.

From figure 5.19 it can be seen that even more problems arise when all fractures are permeable when the fractures are homogenized. This is when compared to figure 5.14. The explicitly represented fractures in the MMs simulations result in an MMs solution that is comparable to the finescale pressure solution. All coarsening ratios seem to have an accurate solution, especially compared to the homogenized test case. In figure 5.20 the pressure can be seen plotted over the chosen trajectory.

Again, the homogenized fracture test case has not resulted in a satisfactory pressure profile. The error compared to the finescale solution for all MMs test cases can be found in table 5.3.5. Every MMs solution with fractures represented explicitly with any coarsening ratio gives a comparable solution with the finescale solution. 


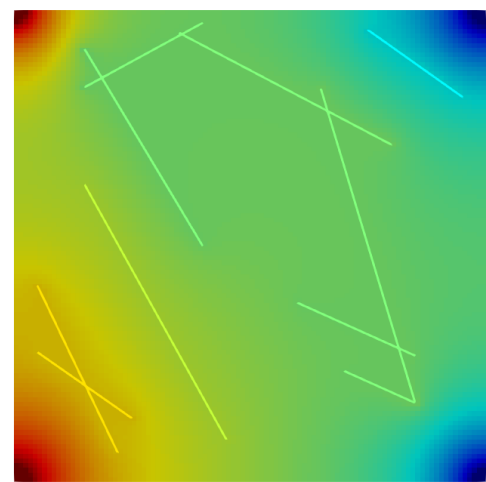

(a) Finescale

$\mathrm{e}=0.48 \%$

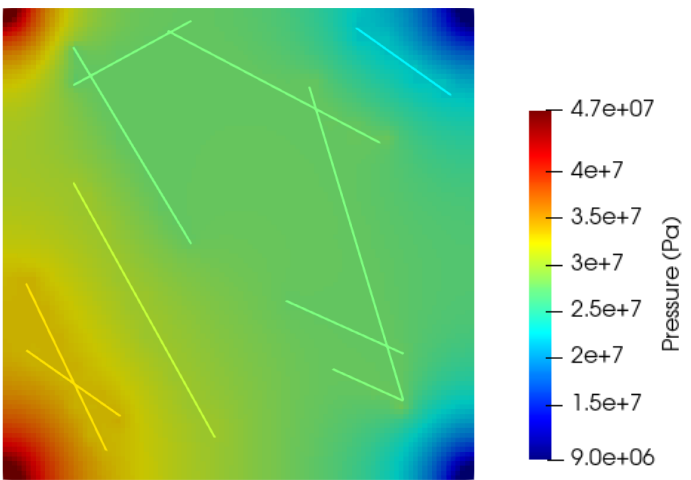

(b) Matrix CR3

Fractures CR3

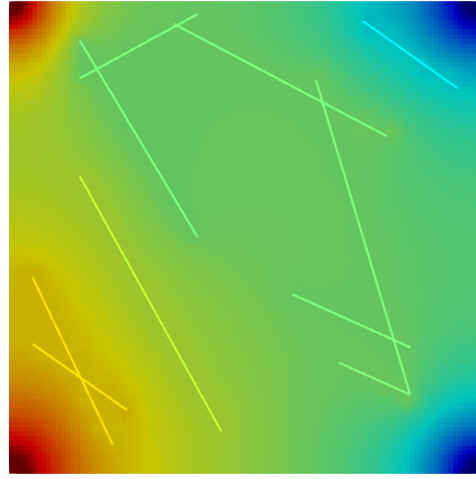

(c) Matrix CR3

Fractures CR9

$\mathrm{e}=0.47 \%$

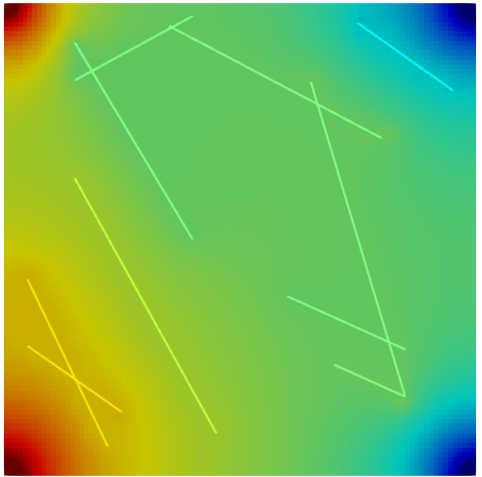

(d) Matrix CR3

Fractures CR63

$\mathrm{e}=0.48 \%$

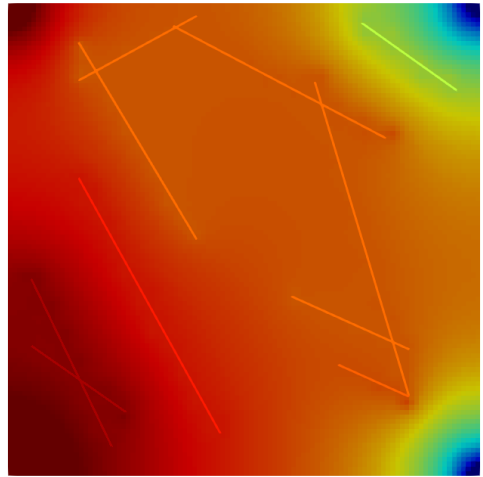

(e) Matrix CR3

Fractures Homogenized $\mathrm{e}=22.08 \%$

Figure 5.19: The figures a-e are a visualization of the single phase pressure solution.

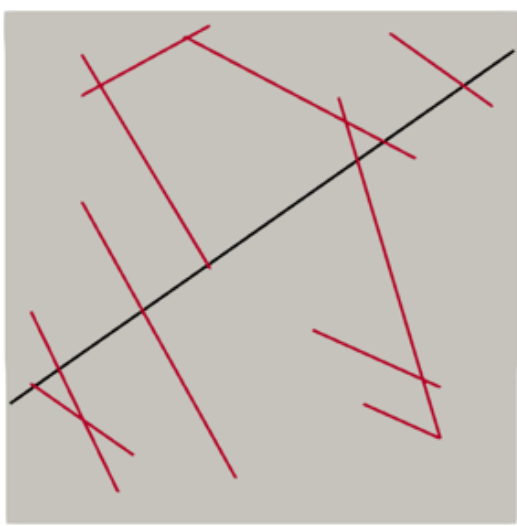

(a)

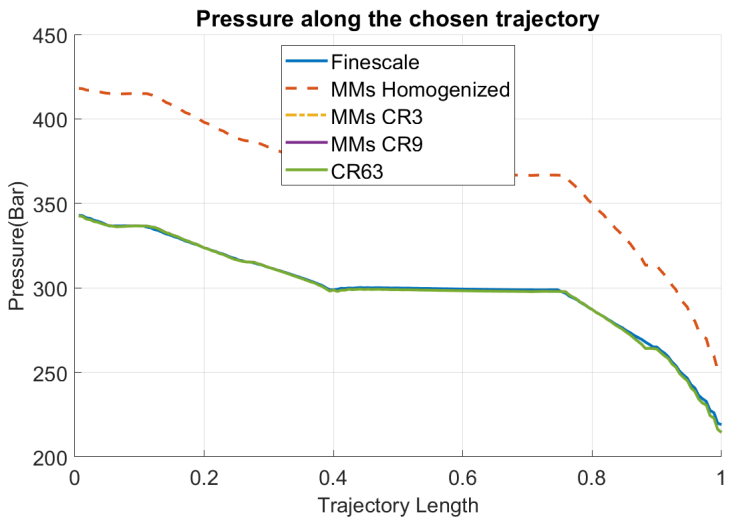

(b)

Figure 5.20: (a) Fracture map with the black line being the trajectory over which the pressure is plotted. (b) The pressure decline belonging to the trajectory shown in (a). The trajectory length is dimensionless. 


\subsubsection{Impermeable Fractures, Horizontal Flow}

In this section the results are presented for a test case in which all fractures are impermeable but are placed according to the benchmark test case by Bernd Flemisch et al. [37]. The fracture setup is shown in figure 5.21 .
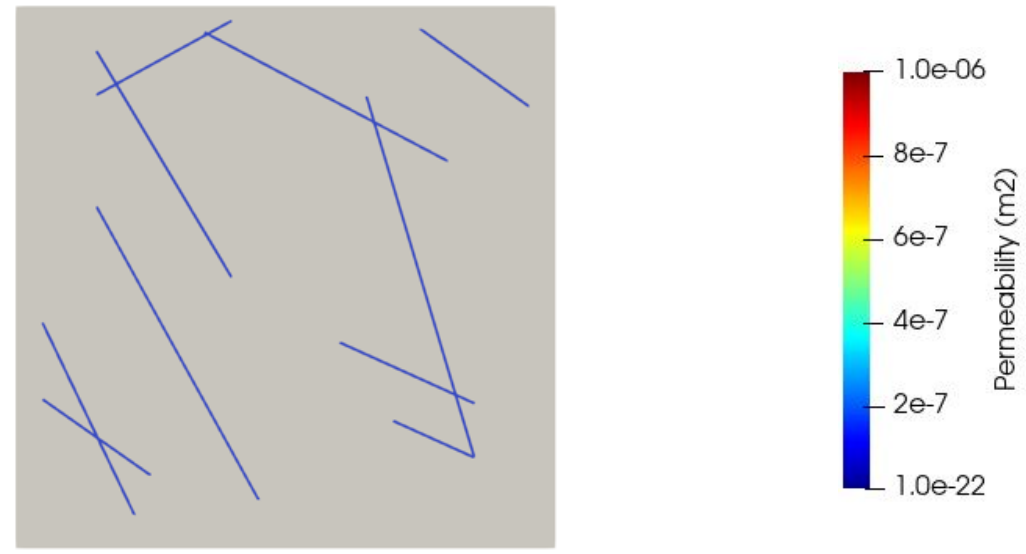

Figure 5.21: The fracture map used for the test cases mentioned below. Fractures with a high permeability are presented in red whereas fractures with a low permeability are presented in blue.

In figure 5.22 the pressure plots can be seen for different coarsening ratios for the fractures and a pressure plot for finescale.

What stands out in figure 5.22 is the high pressure contrasts around the three large fractures in the middel of the plot. This high contrast isn't seen in any of the MMs plots. Eventhough all MMs plots look alike, none of them are able to effectively capture the pressure contrasts. In figure 5.23 the pressure is plotted along the chosen trajectory (shown by the black line).

The pressure plot in figure 5.23 shows what is already visible in figure 5.22. The blue line, the finescale pressure, has significant drops in pressure along the trajectory. These significant drops in the MMs cases are not to be found. The pressure drops gradually for MMs test cases and the steep pressure gradients are not captured. 


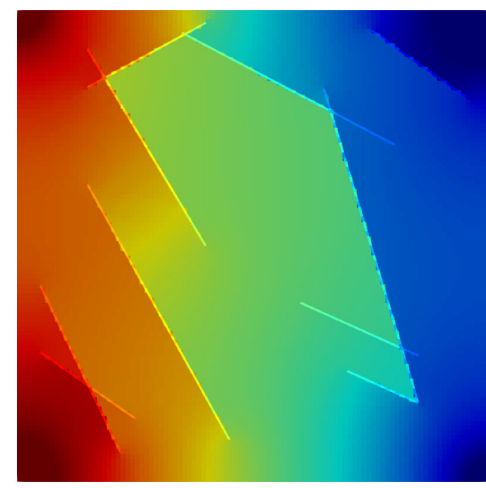

(a) Finescale

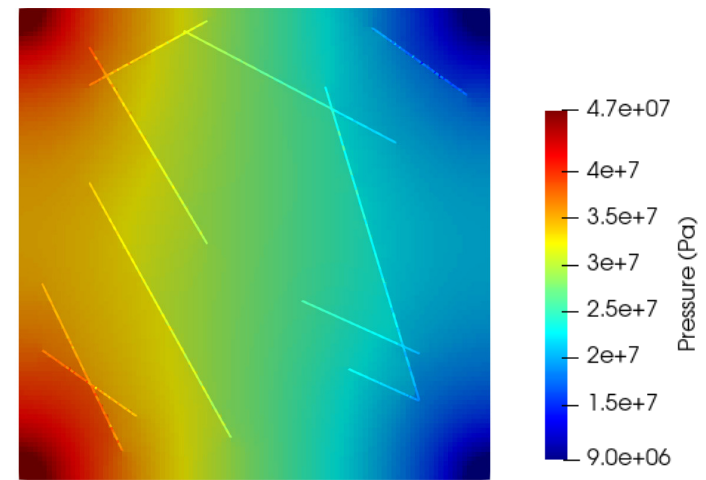

(b) Matrix CR3

$\mathrm{e}=5.86 \%$

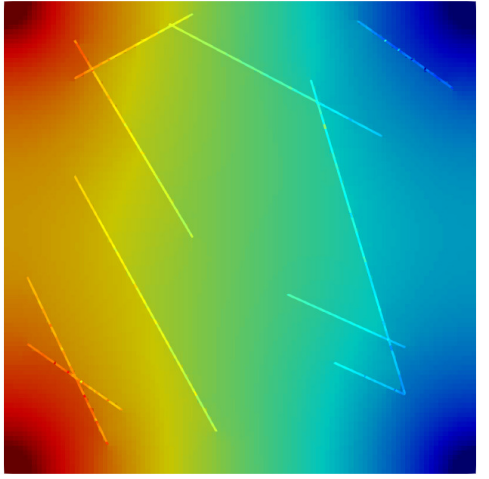

(c) Matrix CR3

Fractures CR9

$\mathrm{e}=5.84 \%$

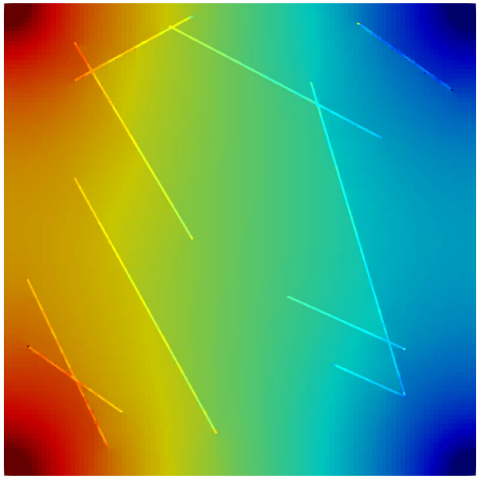

(d) Matrix CR3

Fractures CR63

$\mathrm{e}=5.86 \%$

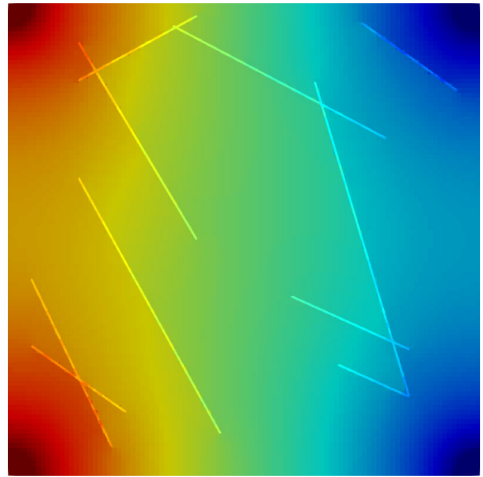

(e) Matrix CR3

Fractures Homogenized $\mathrm{e}=5.89 \%$

Figure 5.22: The figures a-e are a visualization of the single phase pressure solution.

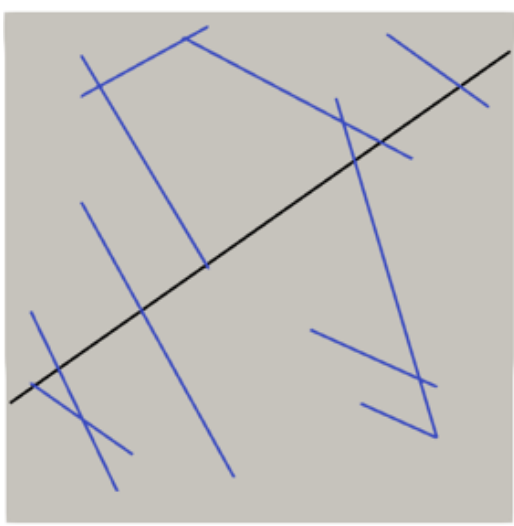

(a)

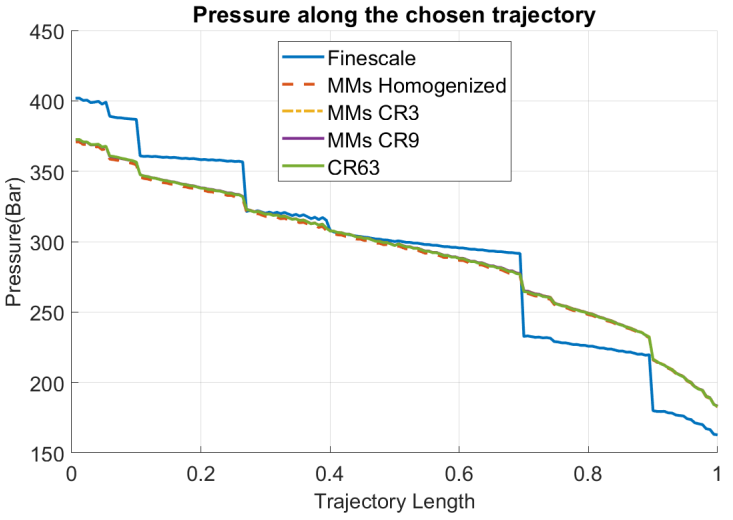

(b)

Figure 5.23: (a) Fracture map with the black line being the trajectory over which the pressure is plotted. (b) The pressure decline belonging to the trajectory shown in (a). The trajectory length is dimensionless. 


\subsubsection{Error Table}

In table 5.3.5 the errors are shown for each MMs pressure solution when compared to finescale. The way this is calculated is shown in the beginning of this chapter.

Table 5.3: The table in which the absolute errors for the first benchmark test case can be found.

\begin{tabular}{|l|l|l|l|l|}
\hline Fracture permeability & $\begin{array}{l}\text { Error } \\
\text { CR 3 (\%) }\end{array}$ & $\begin{array}{l}\text { Error } \\
\text { CR 9 (\%) }\end{array}$ & $\begin{array}{l}\text { Error } \\
\text { CR63 (\%) }\end{array}$ & $\begin{array}{l}\text { Error } \\
\text { Homogenized (\%) }\end{array}$ \\
\hline $\begin{array}{l}\text { Benchmark Settings } \\
\text { Vertical Flow }\end{array}$ & 0.59 & 0.59 & 0.59 & 0.59 \\
\hline $\begin{array}{l}\text { Benchmark Settings } \\
\text { Horizontal Flow }\end{array}$ & 0.67 & 0.66 & 0.66 & 18.29 \\
\hline $\begin{array}{l}\text { All fractures permeable } \\
\text { Horizontal flow }\end{array}$ & 0.48 & 0.47 & 0.48 & 22.08 \\
\hline $\begin{array}{l}\text { All fractures impermeable } \\
\text { Horizontal flow }\end{array}$ & 5.86 & 5.84 & 5.85 & 5.89 \\
\hline
\end{tabular}




\subsection{Benchmark 2 Test Case}

In this section the results belonging to the second benchmark test case will be presented. The test case consists of 6 permeable fractures that split up the matrix in square compartments. The fractures are set up as presented in the paper written by Bernd Flemisch et al [37]. This testcase is run for horizontal flow in which two injector wells are placed at the left corners and two producer wells are placed on the right corners.

The matrix consists of 100 by 100 gridcells, placing the multiscale coarse nodes on th corners according to section 4.3.1.

The fracture setup is shown in figure 5.24.
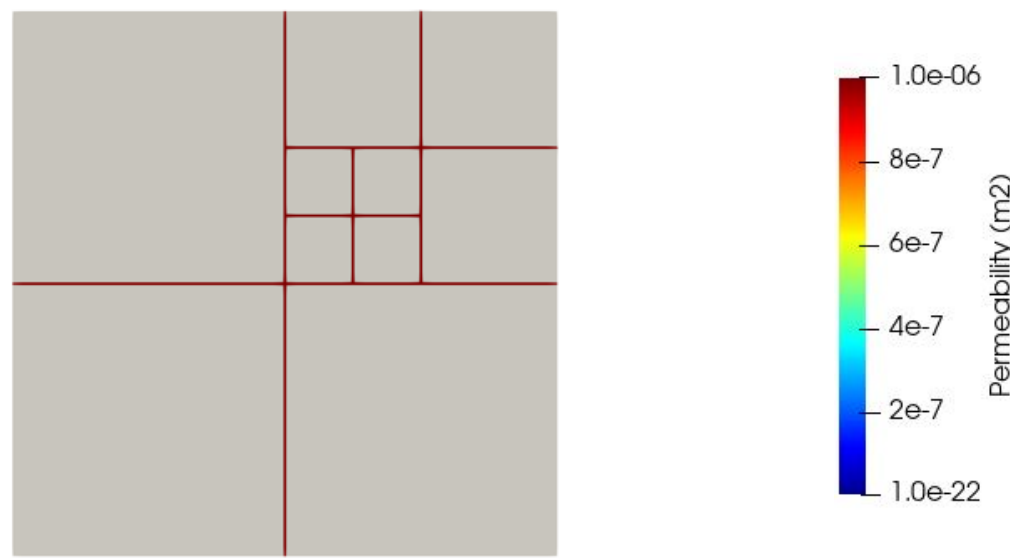

Figure 5.24: The fracture map used for the test case mentioned below. Fractures with a high permeability are presented in red whereas fractures with a low permeability are presented in blue.

In figure 5.25 the MMs results for the different coarsening ratios and finescale results for this test case can be seen.

In figure 5.25, it is easy to see that the test case in which all fractures are homogenized differs most from the finescale solution. Having no coarse nodes on the permeable fractures results in an inaccurate pressure solution. However, the difference between the MMs results with coarse nodes explicitly representing fractures, are not easily visible.

In figure 5.26 the same trend can be seen as in figure 5.25. The test case with homogenized fractures fails to follow the pressure drop visible in the finescale solution, whereas the MMs solutions with explicitly represented fractures are able to show the pressure drop over the chosen trajectory. Between the different coarsening ratios no difference is visible. 


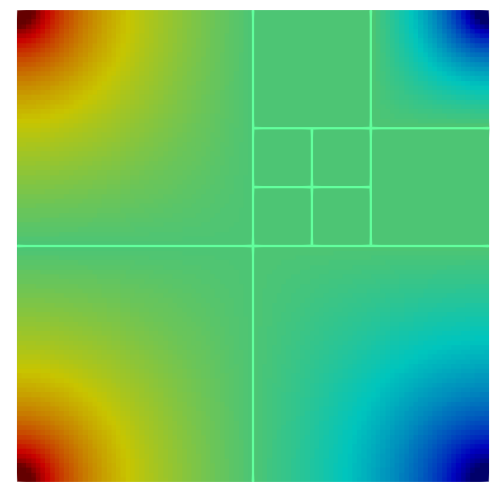

(a) Finescale

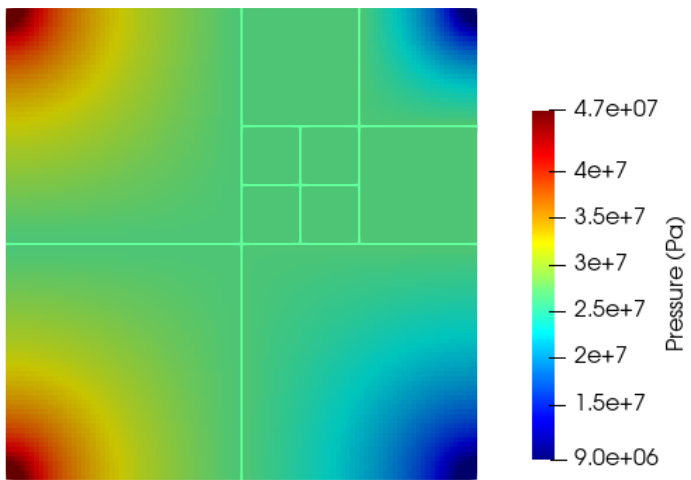

(b) Matrix CR3

Fractures CR3

$\mathrm{e}=0.55 \%$

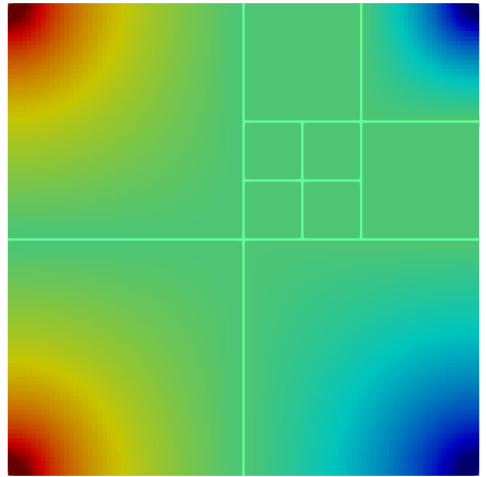

(c) Matrix CR3

Fractures CR9 $\mathrm{e}=0.55 \%$

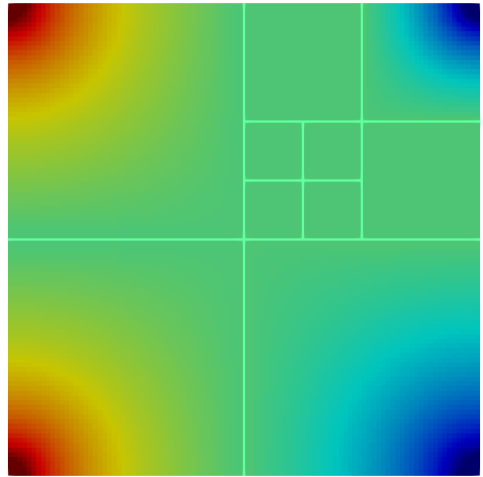

(d) Matrix CR3

Fractures CR63

$\mathrm{e}=0.55 \%$

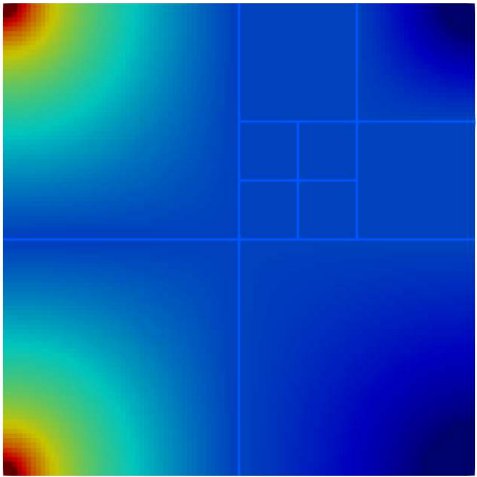

(e) Matrix CR3

Fractures Homogenized $\mathrm{e}=20.83 \%$

Figure 5.25: The figures a-e are a visualization of the single phase pressure solution.

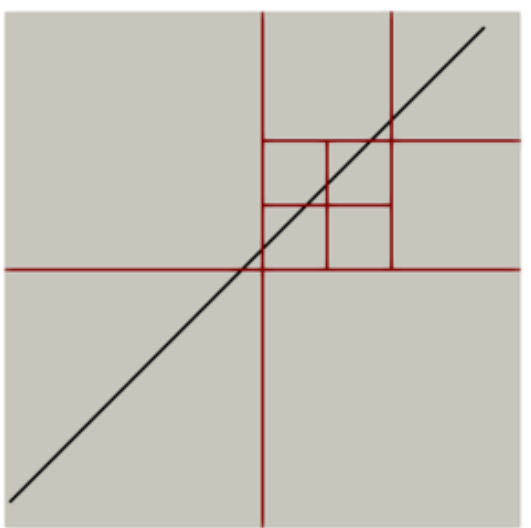

(a)

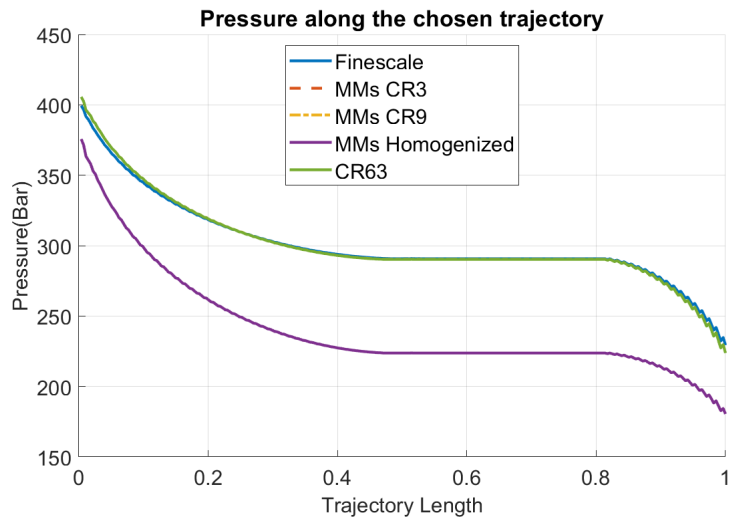

(b)

Figure 5.26: (a) Fracture map with the black line being the trajectory over which the pressure is plotted. (b) The pressure decline belonging to the trajectory shown in (a). The trajectory length is dimensionless. 
Table 5.4: The table in which the absolute errors for the second benchmark test case can be found.

\begin{tabular}{|l|l|l|l|l|}
\hline \multirow{2}{*}{ Fracture permeability } & $\begin{array}{l}\text { Error } \\
\text { CR 3 (\%) }\end{array}$ & $\begin{array}{l}\text { Error } \\
\text { CR 9 (\%) }\end{array}$ & $\begin{array}{l}\text { Error } \\
\text { CR63 (\%) }\end{array}$ & $\begin{array}{l}\text { Error } \\
\text { Homogenized (\%) }\end{array}$ \\
\hline Benchmark Test Case & 0.55 & 0.55 & 0.55 & 20.83 \\
\hline
\end{tabular}

In table 5.4 the error when compared to the finescale pressure solution can be found. The error between the explicitly represented fractures in MMs is the same whereas the homogenized fractures is very high. This shows that homogenizing fractures cannot accurately represent the pressure drop in an MMs simulation.

\subsection{Influence of pEDFM on Results}

In this result section for MMs it is clear that some configurations in MMs simulations work better than others. It is striking that MMs is not able to capture the steep pressure gradients that are caused by impermeable fractures. The MMs solutions all show a gradually declining pressure instead of a sharp drop. Examples of this phenomenon can be found in all test cases with impermeable fractures, especially cases with only impermeable fractures (see section 5.2.2 or 5.3.4). In all these test cases the contrast of the fracture is adjusted, as described in subsection 5.1.1, which can be the origin of this error.

In the following section the MMs simulations for the first benchmark test case in which all fractures are impermeable are presented. However, in the following MMs test case the $\alpha$ factor is not adjusted. This will result in fractures cutting off transmissibility fully in the basis function calculations.

\subsection{Benchmark 1, No Basis Function Adjustments}

In this section the same settings are used to perform an MMs simulation as in section 5.3.4. However, the pEDFM settings for basis functions are not adjusted. For the sake of completeness, the fracture setup is shown again in figure 5.27.
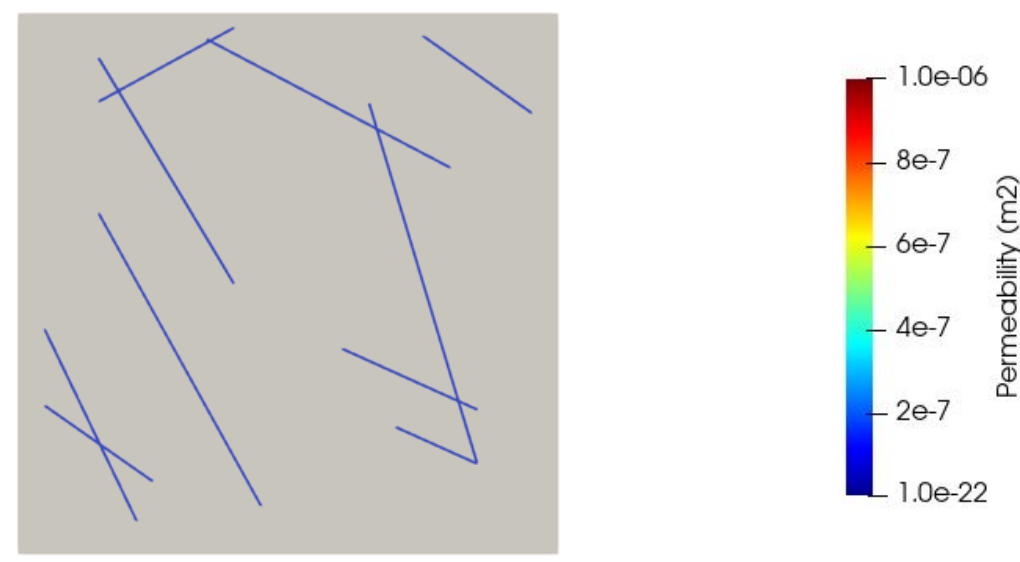

Figure 5.27: The fracture map used for the test cases mentioned below. Fractures with a high permeability are presented in red whereas fractures with a low permeability are presented in blue.

The results for the MMs simulation with the corresponding settings for pEDFM are shown in figure 5.28 . 


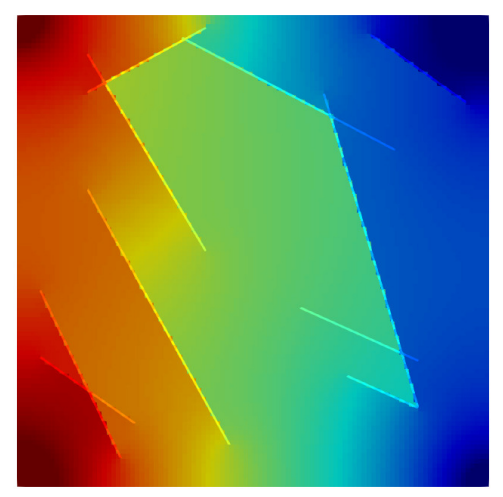

(a) Finescale

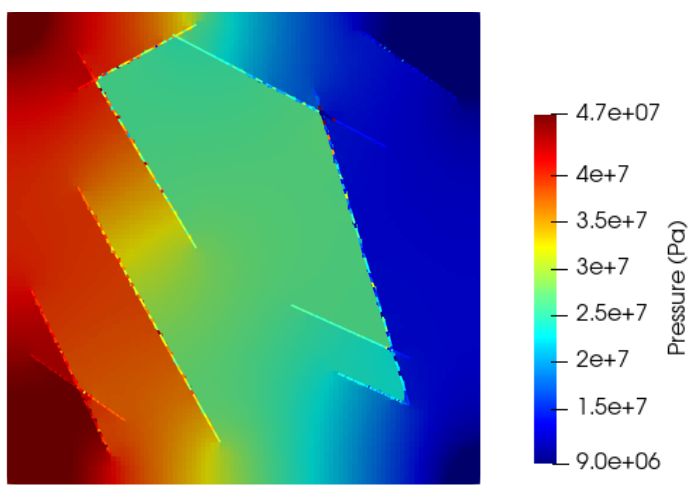

(b) Matrix CR3

Fractures CR3

$\mathrm{e}=38.18 \%$

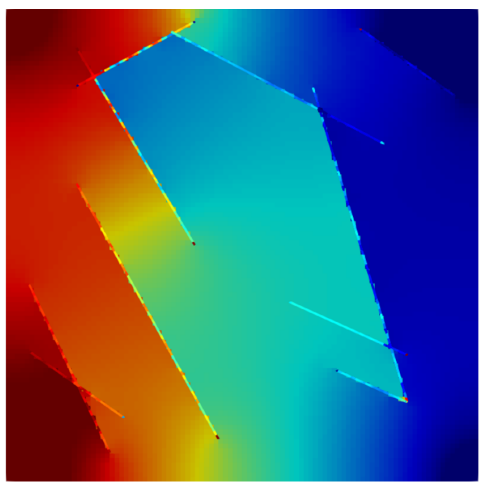

(c) Matrix CR3

Fractures CR9 $\mathrm{e}=9.08 \%$

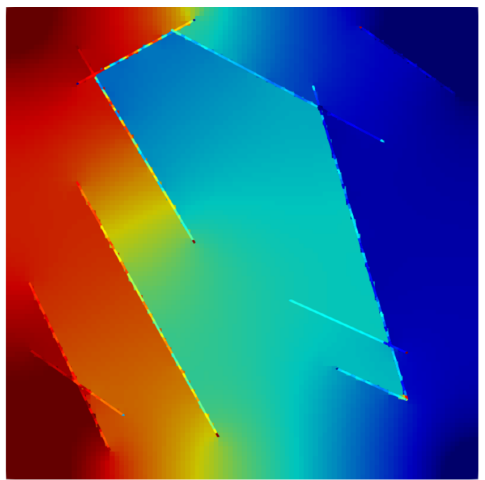

(d) Matrix CR3 Fractures CR63 $\mathrm{e}=9.20 \%$

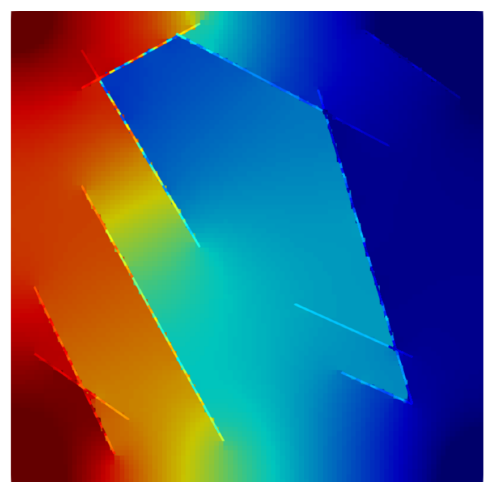

(e) Matrix CR3

Fractures Homogenized $\mathrm{e}=11.78 \%$

Figure 5.28: The figures a-e are a visualization of the single phase pressure solution.

From figure 5.28 it already becomes clear that the results show a greater contrast in pressure and can capture the pressure gradient in more accurately. The gradual change in pressure which could be seen from figure 5.22 has become a sharper pressure drop in using the settings corresponding to this section. This is applicable to all coarsening ratios given to fractures.

In figure 5.29 the pressure is plotted over a chosen trajectory to be able to compare each solution. 


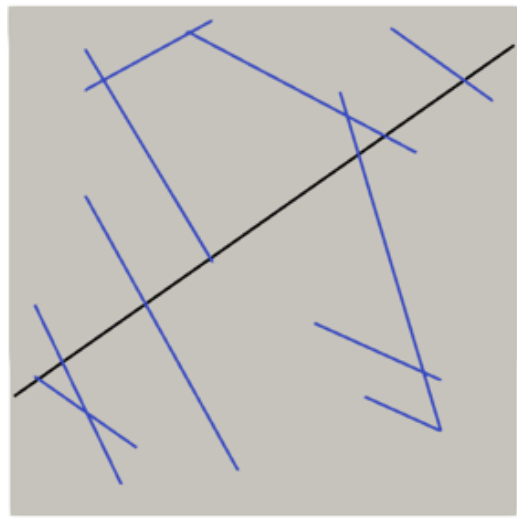

(a)

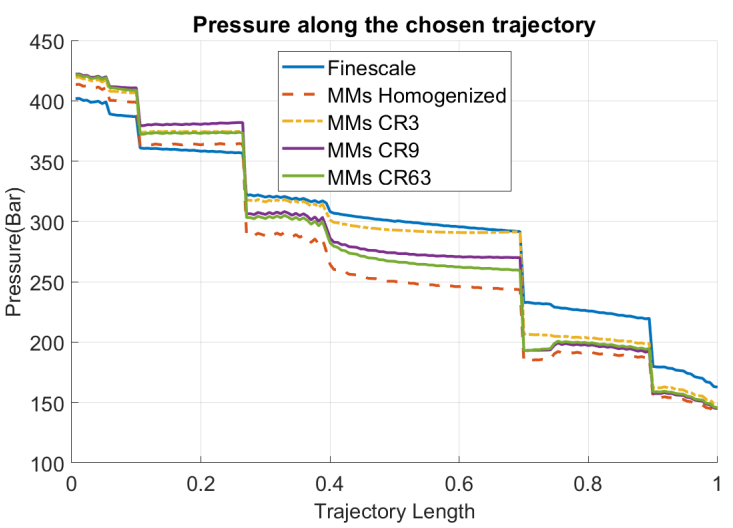

(b)

Figure 5.29: (a) Fracture map with the black line being the trajectory over which the pressure is plotted. (b) The pressure decline belonging to the trajectory shown in (a). The trajectory length is dimensionless.

In figure 5.29 the pressure of each solution is plotted over the shown trajectory. From this plot a clear difference between each coarsening ratio is visible. What is also visible, is that again the sharp pressure gradients are visible from this plot. When looking closely, one can say that the pressure profile belonging to the MMs simulation for fractures with coarsening ratio 3 is most alike to the finescale solution. When figure 5.29 is compared to figure 5.23 the overall pressure profiles deviate more from the finescale solution but are able to capture the pressure gradient better.

In table 5.6 the absolute errors can be found with the corresponding coarsening ratios for fractures with respect to the finescale solution.

Table 5.5: The table in which the absolute errors for the first benchmark test case with corresponding pEDFM settings can be found.

\begin{tabular}{|l|l|l|l|l|}
\hline \multirow{2}{*}{ Fracture permeability } & Error & Error & Error & Error \\
& CR 3 (\%) & CR 9 (\%) & CR63 (\%) & Homogenized (\%) \\
\hline Benchmark Test Case & 38.18 & 9.18 & 9.20 & 11.78 \\
\hline
\end{tabular}

For MMs solutions for fractures with coarsening ratio of 9, 63 and homogenized fractures the absolute error is higher in comparison with the scenario in which the $\alpha$ factor is decreased by 0.01. However, what is most striking is the huge error for the MMs solution for fractures with a coarsening ratio 3. This error percentage is not to be expected with the plot in figures 5.28 and 5.29.

In figure 5.30 the pressure is shown to fit for all values of pressure present in the solution. This way a very small point in the solution becomes visible with a highly negative pressure. This is not realistic and shows a flaw in the MMs approach. When the location of vertices, edges and faces are visualized (see figure 5.30, right), it becomes clear that the fractures are located exactly on top of the vertex where the negative pressure is located. 


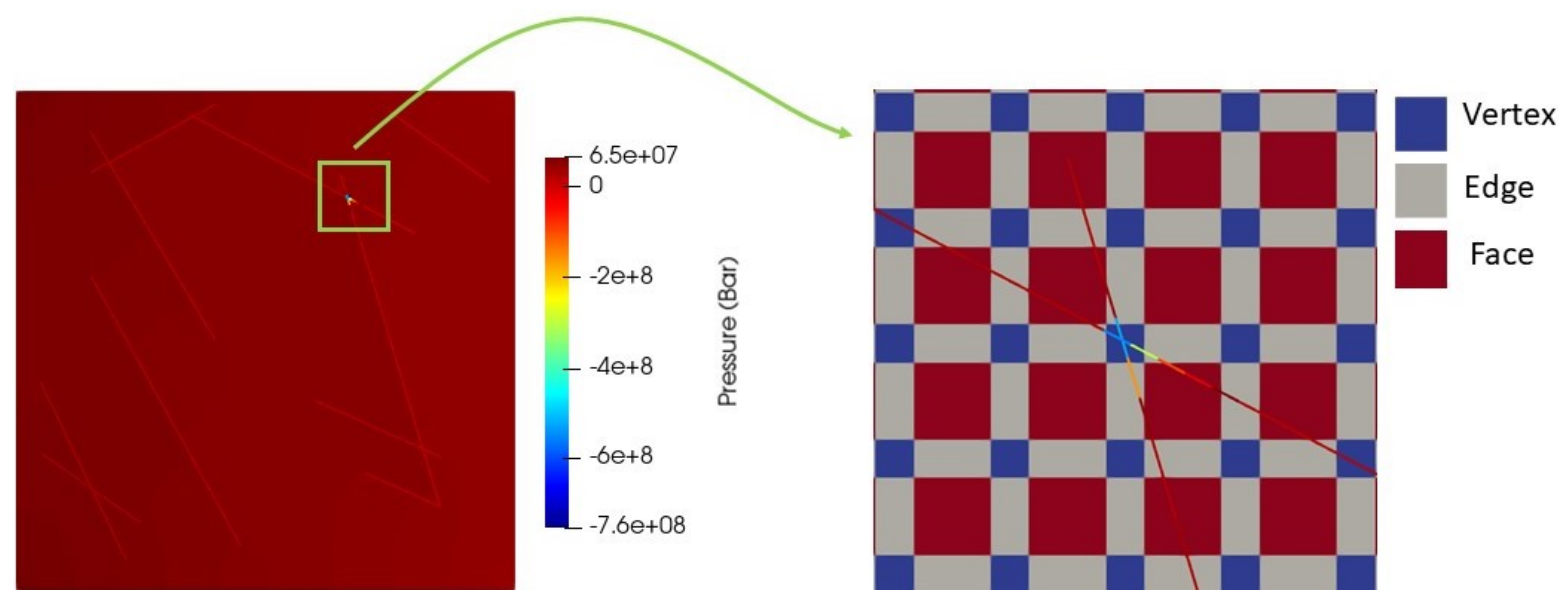

Figure 5.30: A pressure solution for an MMs simulation with fractures given coarsening ratio of 3 and a matrix coarsening ratio of 3 with a pressure scale that has a range to account for all pressures in the solution (left). A visualization of the location of vertices, edges and faces of the matrix but also the location of the fractures (with their pressure solution) for the green box in which negative pressures are present (right).

The same simulation is run for a coarsening ratio of 9 for the matrix. Changing the matrix coarsening ratio will change the location of vertices. The fracture vertices are kept in the same location by keeping the coarsening ratio of 3 .

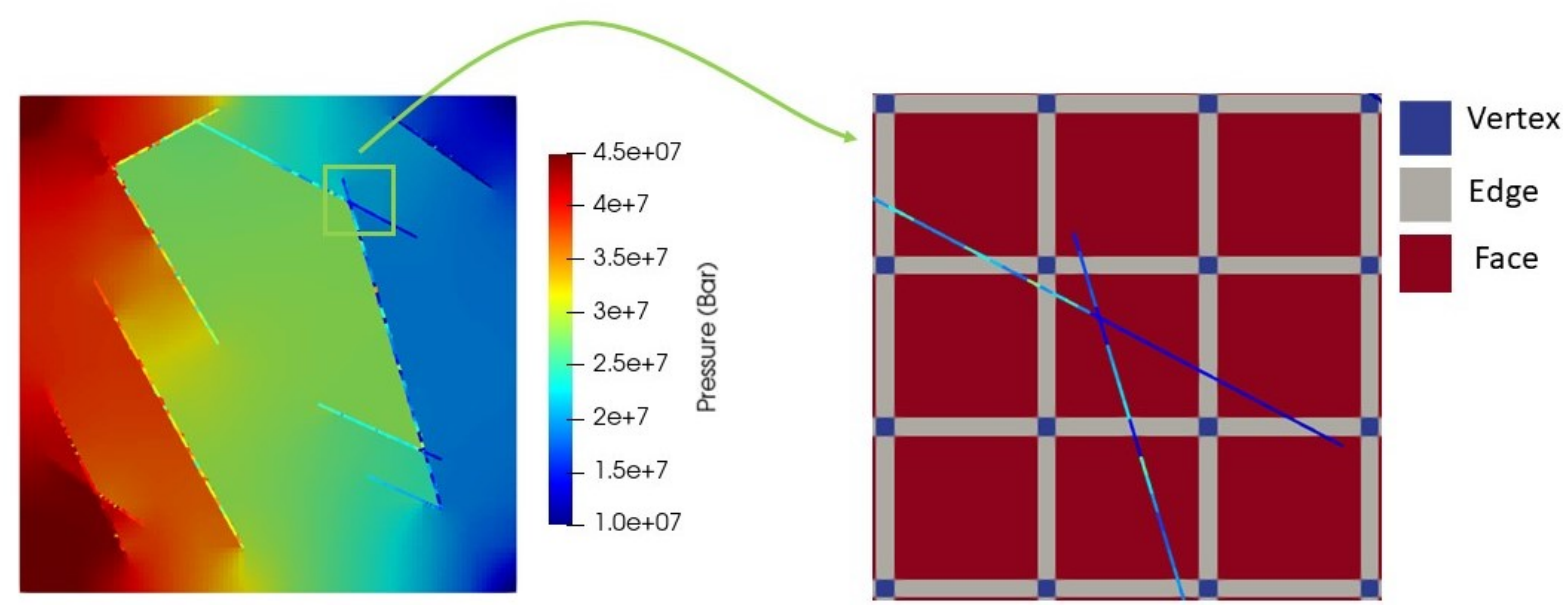

Figure 5.31: A pressure solution for an MMs simulation with fractures given coarsening ratio of 3 and a matrix coarsening ratio of 9 with a pressure scale that has a range to account for all pressures in the solution (left). A visualization of the location of vertices, edges and faces of the matrix but also the location of the fractures (with their pressure solution) for the same green box as in figure 5.30 (right).

What can be seen in figure 5.31 is that the location of the matrix vertices has changed and has moved away from the fractures. This results in a pressure solution without negative pressures and shows how vertex placement for both matrix and fractures can heavily impact the solution. The absolute error for the MMs solution with a matrix coarsening ratio of 9 and a fracture coarsening ratio of 3 is $9 \%$, a significant smaller absolute error when compared with the $38 \%$ belonging to the same simulation with a coarsening ratio of 3 for the matrix.

In chapter 7 the issue on coarse node placement and adjusting basis functions to allow for information leakage is discussed more in depth. 


\section{Chapter 6}

\section{Algebraic Dynamic Modelling Results}

In this chapter the results from the ADM simulations are presented. The test cases are designed to emphasize the capabilities of the implemented methods.

Besides the calculation of the error with respect to the finescale solution, the percentage of active gridcells is also calculated. With the finescale solution as reference, the calculation of active gridcells shows which percentage of finescale gridcells are active to compute the ADM simulation.

\subsection{Simulator Settings}

The test cases discussed in this chapter have all been simulated using the same fracture map. It consists of 30 fractures with high and low permeability with respect to the matrix.
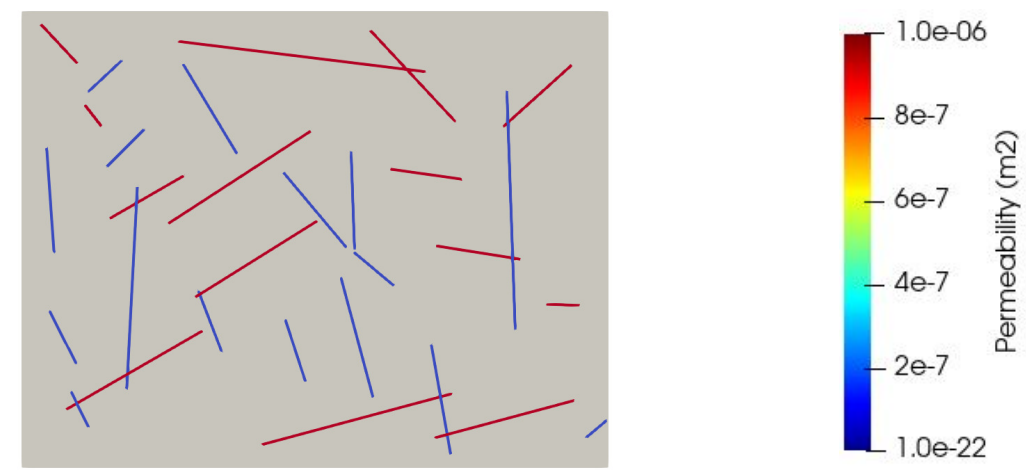

Figure 6.1: The fracture map used for the test cases mentioned below. Fractures with a high permeability are presented in red where fractures with a low permeability are presented in blue.

The reservoir has a size of $(244 x 190)$ gridcells and is $(90 x 70)$ meters. This makes each gridcell roughly $0.35 \mathrm{~m}^{2}$. The reservoir is homogeneous with a permeability of $10^{-14} \mathrm{~m}^{2}$. Two injector wells are placed in the left corners $\left(0,0\right.$ and $\left.0, N_{y}\right)$. Two producer wells are placed in the right corners $\left(N_{x}, 0\right)$ and $\left(N_{x}, N_{y}\right)$. The run time for each simulation is 200 days. In table 6.1 the reservoir properties that do not change throughout all simulations are given. 
Table 6.1: Reservoir properties that stay constant throughout all simulations presented in this chapter.

\begin{tabular}{|l|l|l|}
\hline Reservoir Property & Value & Unit \\
\hline Porosity & 0.2 & - \\
\hline Fluid Density Phase 1 & 1000 & $\mathrm{~kg} / \mathrm{m}^{3}$ \\
\hline Fluid Density Phase 2 & 800 & $\mathrm{~kg} / \mathrm{m}^{3}$ \\
\hline Viscosity Phase 1 & $3 \mathrm{e}-3$ & $\mathrm{~Pa} \mathrm{sec}$ \\
\hline Viscosity Phase 2 & $1 \mathrm{e}-1$ & $\mathrm{~Pa} \mathrm{sec}$ \\
\hline Compressibility Phase 1\&2 & 0 & $1 / \mathrm{Pa}$ \\
\hline Reservoir Permeability & $1 \mathrm{e}-14$ & $\mathrm{~m}^{2}$ \\
\hline Injection Well Pressure & $5 \mathrm{e} 7$ & $\mathrm{~Pa}$ \\
\hline Production Well Pressure & $1 \mathrm{e} 7$ & $\mathrm{~Pa}$ \\
\hline Fracture Aperture & $5 \mathrm{e}-3$ & $\mathrm{~m}$ \\
\hline
\end{tabular}




\subsection{Flexible Coarsening Ratios for Fractures}

In this section it is investigated how the changing of coarsening ratios for fractures influences the accuracy and efficiency of the simulation. The changing of coarsening ratios changes the number of vertices on the fractures. With more vertices on a fracture one can expect the simulation to be more accurate. However, with more vertices on the fractures, one can also expect that the active amount of grid cells will be higher.

Three different scenarios are run to verify these assumptions. All simulations in this test case are given 1 level of ADM coarsening. Fractures are given a fixed amount of grid cells. The fractures are given 46 grid cells with 3,9 and 15 coarsening ratios in each scenario. Giving a fracture 46 grid cells with a coarsening ratio of 3 will ensure the fracture has 16 vertices. The same goes for coarsening ratios 9 and 15, giving 6 and 4 vertices respectively. This is according to equation 4.3.1.

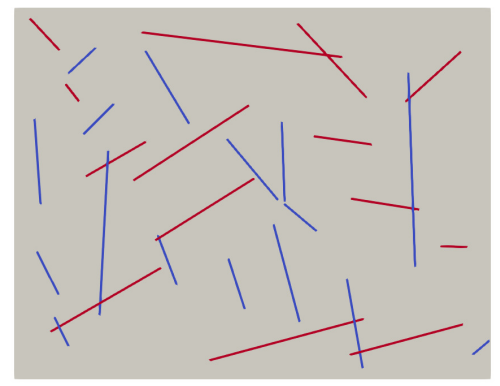

(a) Fracture map

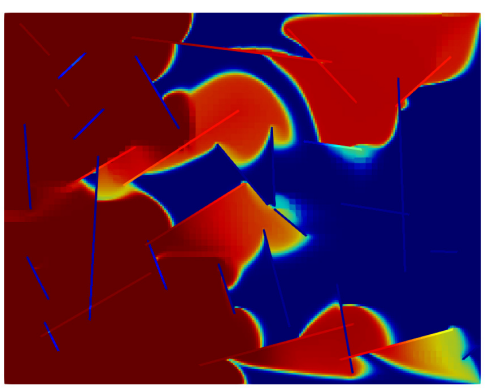

(c) Matrix CR3 Fracture CR3
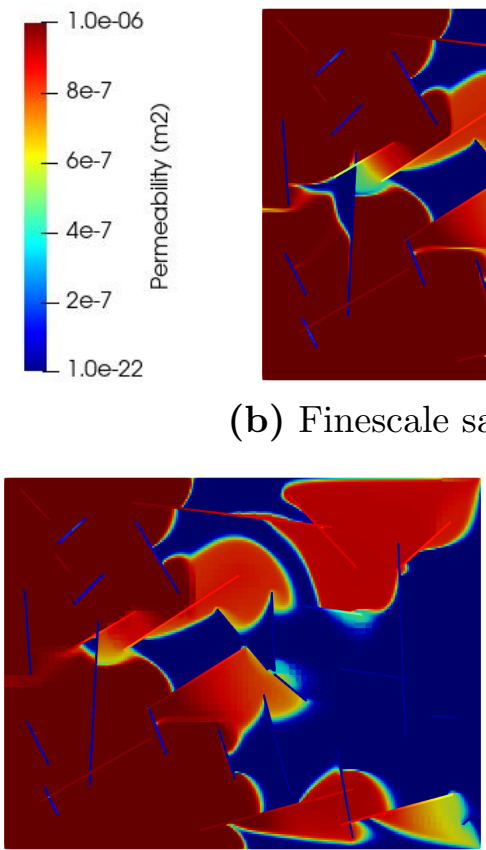

(d) Matrix CR3 Fracture CR9
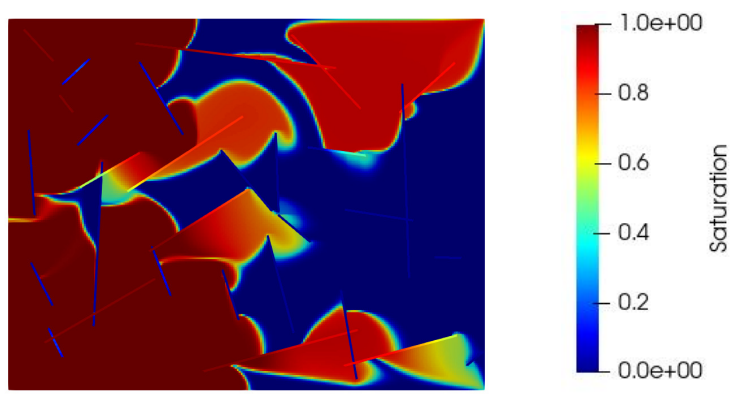

(b) Finescale saturation solution

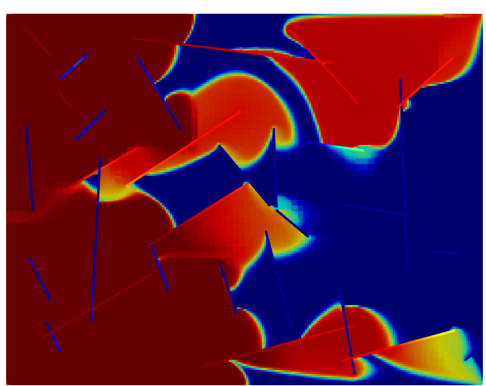

(e) Matrix CR3

Fracture CR15

Figure 6.2: The figures b-e are a visualization of the reservoir after 150 days of injection and production. This is at time-step 75 as each time-step is set to be 2 days, with CR denoting coarsening ratio.

The results of the different coarsening ratios can be seen in figure 6.2. The error of the solutions when compared to the finescale solution can be seen in figure 6.3. The results regarding pressure can be found in appendix C.1.

In figure 6.3 it can be seen that changing the coarsening ratios marginally impacts the simulation. The grid refinement scheme is used in all simulations to detect the front, this is leading for the amount of active grid cells. This means the percentage of grid cells used is not much influenced by the coarsening ratios but much more by the front detection scheme.

In figure $6.3 \mathrm{~b}$ and $6.3 \mathrm{c}$, the error of the $\mathrm{ADM}$ solutions with respect to the finescale solution are very similar. This can also be explained by the grid refinement scheme as the areas with a high gradient in pressure or saturation are covered by finescale grids. These areas with a high gradient 


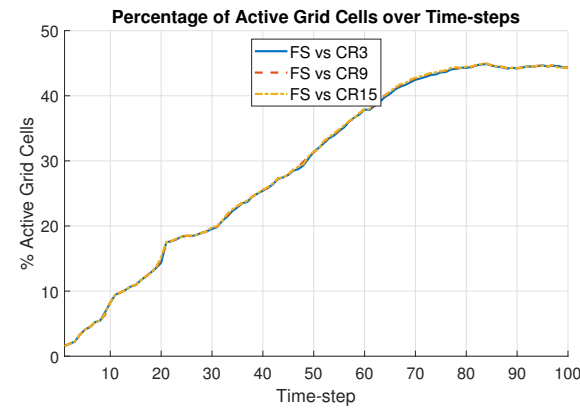

(a)

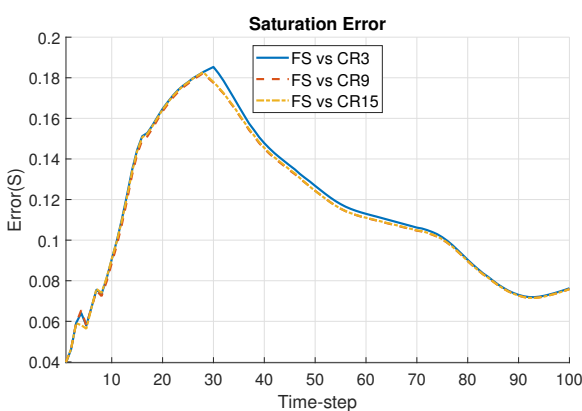

(b)

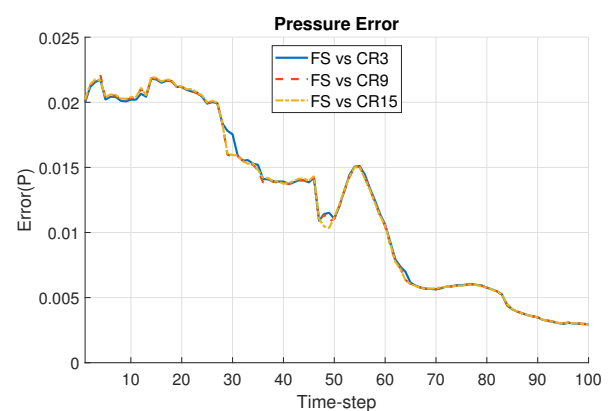

(c)

Figure 6.3: (a) Percentage of active grid cells with CR denoting coarsening ratio for fractures as the matrix coarsening ratio is kept equal throughout the simulations. (b) Saturation error (c) Pressure error

in pressure or saturation are the areas that are the origin of most of the error in ADM solutions but solving exactly these areas using a finescale grid reduces the error. It is important to note that the simulations run for this section have all the fractures coupled to the matrix regarding the grid refinement scheme. This ensures in that any areas with a high gradient in pressure or saturation a finescale solution is used.

\subsection{Coupling of Fractures}

In this section the results are presented for the implementation of the coupling of fractures to the grid refinement scheme. The described method in section 4.5 introduces the coupling of the fracture grids and matrix grid when condition 4.5.2 is considered.

To test what influence the coupling of fractures has, 3 levels of coarsening have been used. Three different scenarios are considered. The first scenario is the scenario where no fractures are coupled (i.e. decoupled). In the second scenario all fractures are coupled and in the last scenario the fractures with a high permeability are coupled.

The amount of gridcells used for the simulation at each timestep is plotted in 6.5. Besides this, the error with respect to saturation and pressure is plotted. By coupling the fractures to the error for both saturation and pressure is reduced. The pressure error drops the most in comparison to the decoupled simulation. However, the reduction of error by coupling fractures does come with a higher computational cost. The percentage of active gridcells does increase for a fully coupled simulation. Only coupling highly permeable fractures results in having a comparable amount of active grid cells but still reduces the amount of gridcells drastically. 
The saturation of a fracture with a low permeability will only increase very slowly and after a long period of injection. This can be seen in figure 6.4. In the resulting image for finescale solution is after 150 days of injection. Still, some fractures have a very low saturation of the injected phase. This is because the fluid has a lot of difficulty flowing through the fracture. It is not necessary to capture a fracture with such low permeability that flow through it is nearly not possible, and the flooded surrounding matrix in a finescale resolution. This is why the number of gridcells can be reduced by decoupling low permeable fractures and obtain a comparable error for both saturation and pressure.

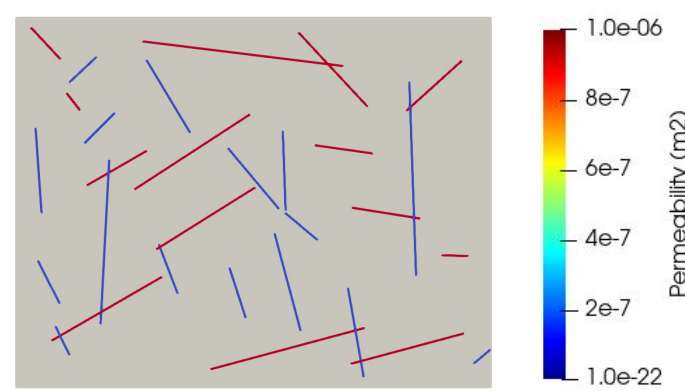

(a) Fracture map

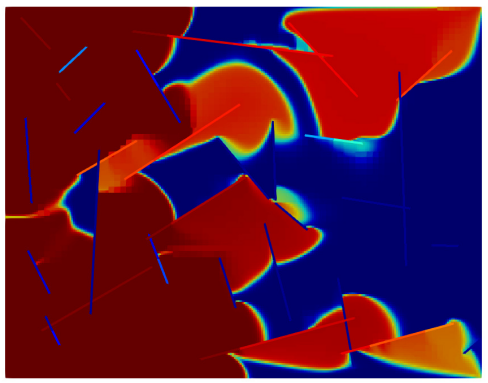

(c) Fractures decoupled

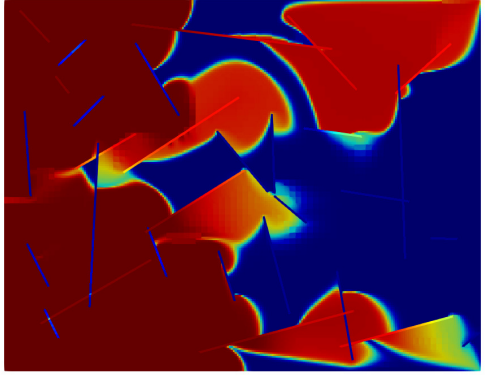

(d) All fractures coupled

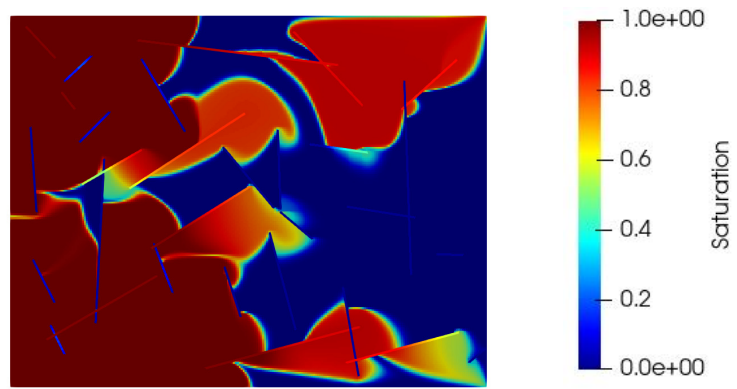

(b) Finescale saturation solution

Figure 6.4: The figures b-e are a visualization of the reservoir after 150 days of injection and production.

In figure 6.6, the gridding used in the simulation is plotted over the saturation solution. By plotting the grids used in the simulation an insight can be given into how coupling fractures to the grid refinement condition effects the solution. In $6.6 \mathrm{c}$, the saturation in fracture cells is not taken into account when the simulator looks at the refinement condition. An example of this can be seen in the top left corner where a low permeable fracture has a low saturation of the injected phase compared to the surrounding matrix and the gridding around it is not in finescale. The fully coupled solution which can be seen in figure 6.6, does have this same area computed with a finescale grid. In fact, all fractures with a low permeabilty are surrounded by finescale grids. By only coupling the high permeable fractures, fluid flow happening through these fractures into the matrix is perfectly captured. If these were to be decoupled, the front would still be captured but much slower as it would only be detected when it flows from matrix to matrix cells whereas the front is now already detected when fluid flows from the fracture into the matrix. The results regarding pressure can be found in appendix C.1. 


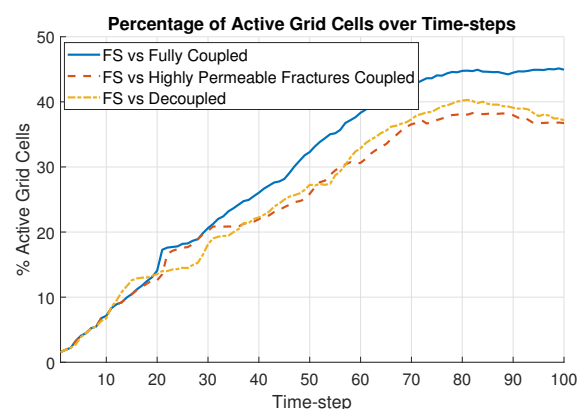

(a)

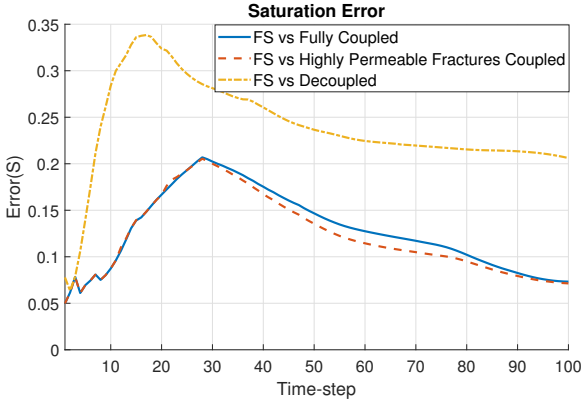

(b)

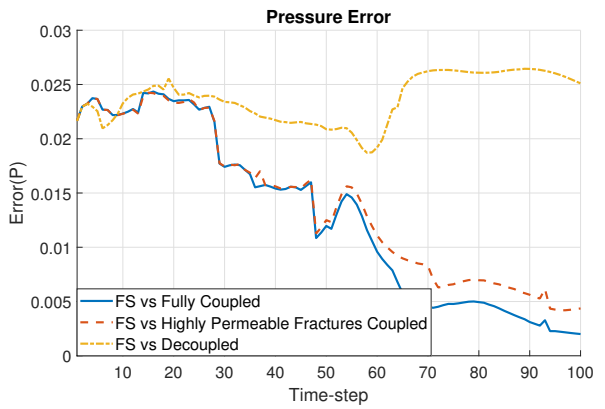

(c)

Figure 6.5: (a) Percentage of active grid cells (b) Saturation error (c) Pressure error

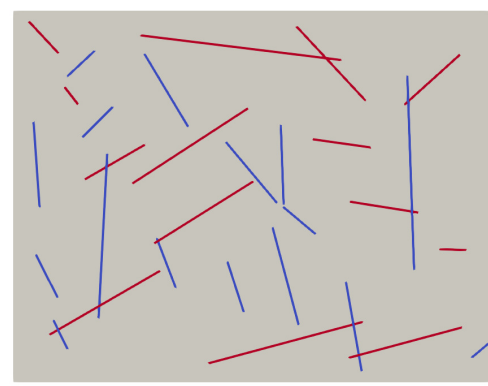

(a) Fracture map

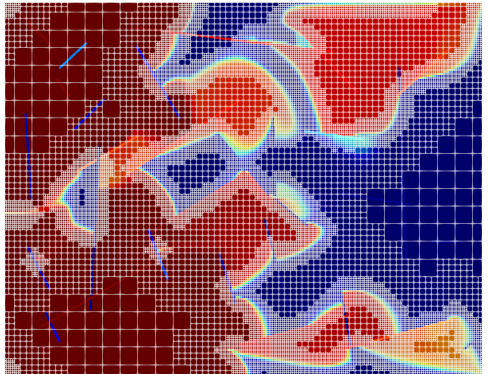

(c) Fractures decoupled
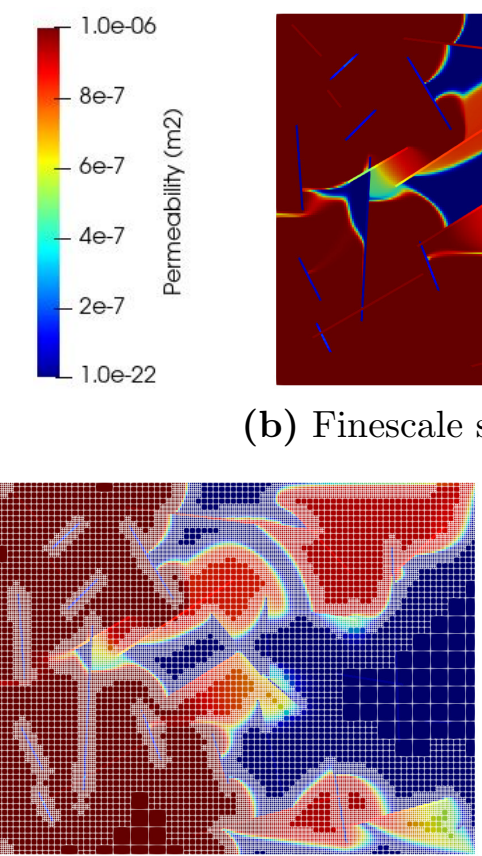

(d) All fractures coupled

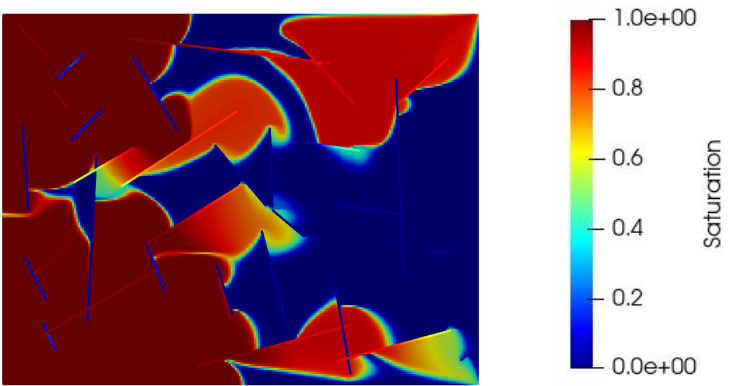

(b) Finescale saturation solution

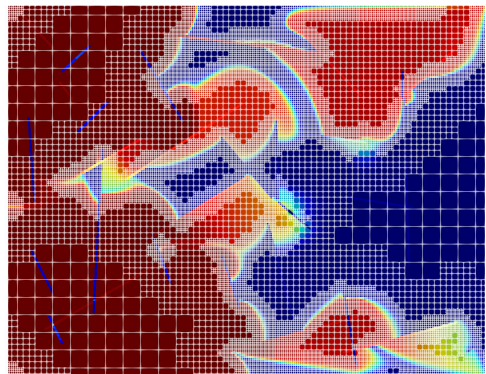

(e) Permeable fractures coupled

Figure 6.6: The figures b-e are a visualization of the reservoir after 150 days of injection and production. The gridding of the reservoir is plotted over the saturation solution to provide insight in how the front is captured by the grid refinement scheme. 


\subsection{Field Scale Sensitivity Analysis}

In the following two subsections the field applicability is shown. Besides this, the sensitivity of the finescale solution is investigated to changes in principle stress direction. This shows how the error of the multiscale can be put into perspective when it comes to geological uncertainty.

\subsubsection{Field Scale Applicability}

In all of the results shown before in respectively chapter 5 and 6 this research shows how using a multiscale solution can be applied to relatively simple test cases. In this section results are presented when using the field scale test case with a fracture map that is stress dependent as presented in chapter 2.

The fracture map used is derived from the methodology described in chapter 2. It consists of fractures that are considered to be hydraulically active and fractures that are not. The more the orientation of a fracture differs from being critically stressed, the lower the permeability assigned to that fracture will be.

The same simulator settings are being used as mentioned in 6.2 but now for a much larger amount of fractures (447). This means a multiphase simulation with four wells (2 injectors, 2 prducers) is performed. The finescale solution is again compared to the multiscale solutions. In this section the results belonging to the pressure solutions will be compared.

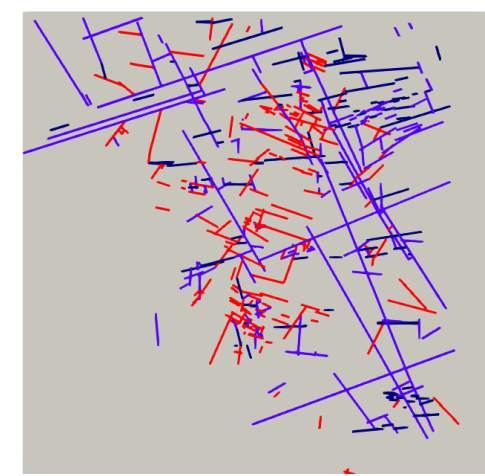

(a) Fracture Map

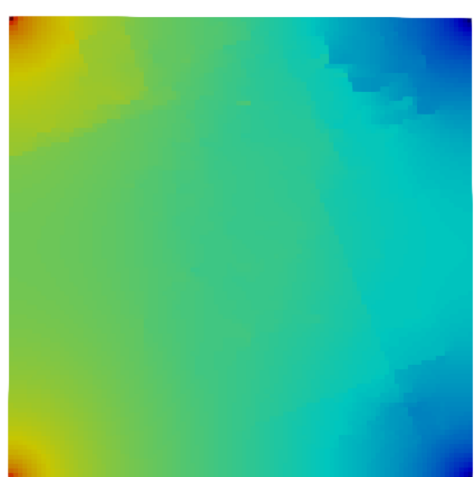

(c) Matrix CR3

Fractures CR3 $\mathrm{e}=0.8 \%$
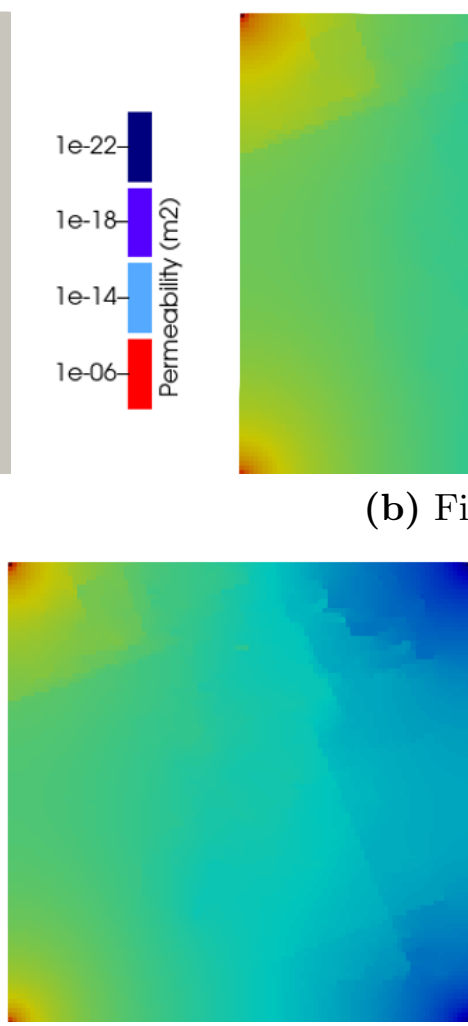

(d) Matrix CR3

Fractures CR6 $\mathrm{e}=6.0 \%$

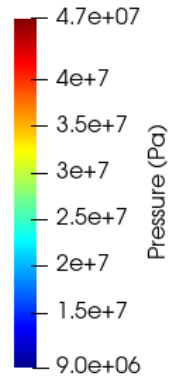

(b) Finescale

Figure 6.7: The figures b-e are a visualization of the multiphase pressure solution at time step 1 (i.e. 1 day of injection). To be able to compare the solutions clearly, the fractures have not been shown in the figures b-e. The fractures present can be seen in the fracture map, figure a. 
With coarsening ratios 3,6 and 36 a total of 13,7 and 2 vertices are placed on each fracture respectively. From the error we see a very accurate result for the coarsening ratio of 3 . The accuracy of coarsening ratio 6 and 36 is comparable. This shows that the multiscale solutions can also be used in a field scale test.

\subsubsection{Sensitivity to Geological Uncertainty}

So far this research has compared a finescale solution with a corresponding multiscale solution. However, the pressure solution belonging to the field scale case is not only dependent on the simulation strategy applied. The solutions belonging to the field scale solutions are dependent on geology too. That is why in this section not only the finescale solutions are compared to the multiscale solutions but also to a changing direction of subsurface stress. The fracture map used is based on this direction and through this sensitivity test the results will show how the finescale solutions can be compared with each other for a different stress direction.

By changing the direction of principle stress by 45 degrees, the range of fractures that are critically stressed will change too. This has an impact on the fracture map used for single and multiphase simulations.

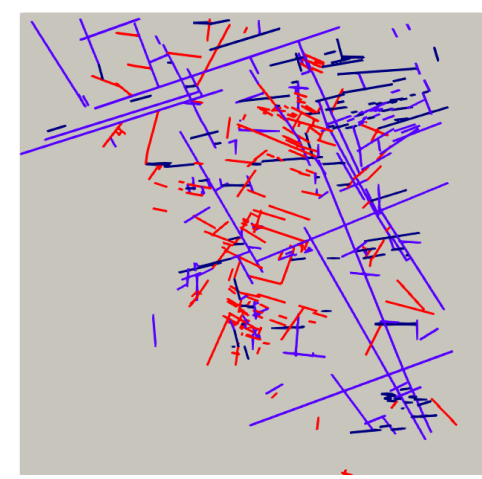

(a) Fracture Map N-S stress

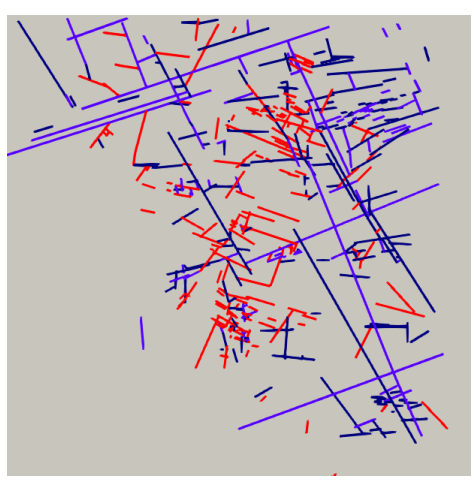

(c) Fracture Map

NE-SW stress
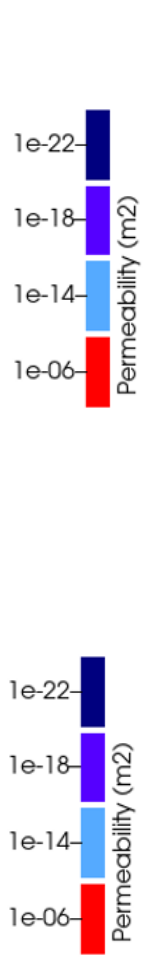

(d) Finescale NE-SW stress $\mathrm{e}=3.9 \%$

Figure 6.8: The figures $\mathbf{b} \& \mathbf{d}$ are a visualization of the multiphase pressure solution at time step 1 (i.e. 1 day of injection). To be able to compare the solutions clearly, the fractures have not been shown in the figures b-e. The fractures present can be seen in the fracture map, figure a \& $\mathbf{c}$.

In the figure 6.8 the results belonging to two different stress settings can be seen. As the stress differs, the result for the multiphase finescale solution differs too. The error mentioned below figure 
$6.8 \mathrm{e}$, is the difference in pressure between the finescale solution belonging to a N-S stress.

As can be seen from the results mentioned in figure 6.7, the error of changing the stress direction is very comparable to using a multiscale method. One way of visualizing this can be done by applying the same setup as used in chapter 5 . The pressure across a chosen trajectory can be plotted for both the finescale solutions and a multiscale solution.

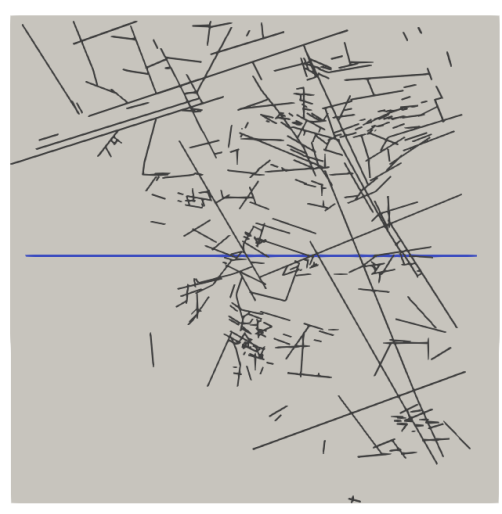

(a)

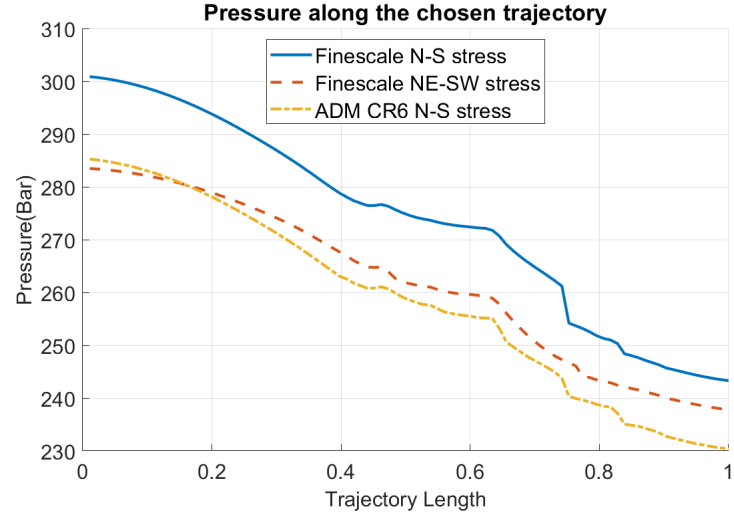

(b)

Figure 6.9: (a) Fracture map with the blue line being the trajectory over which the pressure is plotted. The fractures are not shown with their corresponding permeabilities as this differs for certain pressure declines. This plot only shows the location of fractures and the trajectory chosen over which to plot the pressure decline. (b) The pressure decline belonging to the trajectory shown in (a). The trajectory length is dimensionless.

In figure 6.9 , it is visualized how the multiscale solution belonging to a N-S stress direction is very comparable to the finescale solution belonging to a NE-SW stress. This shows how a change in stress direction also has a significant influence on the pressure distribution and thus flow through the reservoir. 


\section{Chapter 7}

\section{Conclusion and Discussion}

In this chapter the most important findings of the research are summed up and discussed. Not only the work done for this research is discussed but also recommendations for future research are presented.

\subsection{Conclusion}

Through the work done in this research it is possible to make a few conclusions. In chapter 2 a method is presented in which fractures are roughly classified. According to methods described by Mark Zoback [12] and Richard Allmendinger [13] a simplistic method is built to distinguish conductive fractures from blocking fractures based on principle stresses and fracture orientation. Not only are the fractures classified, they are also given a realistic location based on outcrops and geological interpretations. This is done with the help of the work performed by Pierre Bruna and Kevin Bisdom [8] [3].

Using the methods described in chapters 3 and 4 meaningful simulations have been performed showing how one can simulate single- and multiphase injection using MMs and ADM. The flexible coarsening for fractures is demonstrated to be useful to keep multiscale solutions accurate and to decrease computational costs. The user can choose a matrix coarsening ratio for both MMs and ADM simulations and use results from this research to give the minimal coarse nodes necessary to fractures to maintain an accurate solution. Assigning no coarse nodes to a fracture (i.e. homogenizing) is not advised based on the results from chapter 5 as this does not result in an accurate solution.

Both chapter 5 and section 6.2 , show that to be sure to have an accurate solution, fractures need to be given at least 2 coarse nodes (i.e. one at each fracture tip). In chapter 5 it is shown that fractures with blocking characteristics are represented accurately without coarse nodes at all in a static MMs pressure simulation. From chapter 6 it is important to note that the number of active gridcells (in other words the computational costs throughout the simulation) is dominated by the grid refinement scheme and the flexible coarsening ratios for fractures and matrix play a less important role in accuracy and efficiency.

A more important role in ADM simulation is given to the coupling of fracture gridcells to matrix gridcells with respect to the grid refinement scheme. When fracture gridcells are coupled a more accurate solution is acquired but a price is paid in terms of computational costs. Considering cases in which all fractures are coupled to the matrix, a higher percentage of active gridcells is used. When only conductive fracture cells are coupled, a solution is obtained that is close to a fully coupled solution. However, only coupling fracture cells belonging to conductive fractures reduces the percentage of active gridcells drastically. This combination of accuracy and efficiency results in only coupling conductive fractures to be favorable when using ADM. 
Finally, this research shows that the proposed methods can be used in a field scale test case. By changing the direction of the principle stress this research demonstrates the importance of the geological uncertainty. It is important to keep in mind that all multiscale simulations are compared with a finescale solution. This finescale solution however, is not the true subsurface scenario as an analogue fracture map is used with an assumed direction of stress.

\subsection{Discussion}

Through this section the shortcomings of this research are addressed. Firstly the simplistic approach of the fracture classification can be discussed. From this section it is easy to see that a lot of improvement can be made in this field. However, the challenge of locating and classifying all fractures present in a reservoir is still huge and very complex. When one wants to compare simulation results with a possible true solution of injecting and producing from the scenarios simulated in this research, one should realize that the biggest error doesn't lie within the simulation strategy, coarsening ratios or fracture upscaling but in the placement and characteristics of the fractures in the used fracture input. This is the geological uncertainty that many commercial and scientific simulators have to face when simulating flow through subsurface reservoirs.

This brings us to the second section that needs to be discussed. All scenarios presented in this research consider using a homogeneous reservoir. This simplifies nature drastically as no reservoir in the subsurface will be this homogeneous. Besides the permeability characteristics of the matrix being simplified it is easy to see that a $2 \mathrm{D}$ reservoir with $1 \mathrm{D}$ fractures is a drastic simplification of reality. For future research it would be nice to see how the approach used in this research performs in a heterogeneous model. Besides this, ignoring gravity forces and assuming incompressibility simplifies nature.

In section 4.3 the methodology of calculating basis functions is explained. Here it is mentioned that the basis functions are a solution of a localized problem. However, when fractures are crossing the boundaries of this localized problem, the information (i.e. the basis function) leaks out of the dual coarse grid. This enlarges computational costs for calculating the basis functions and the localized solutions are not localized any more. This can be considered a problem and a solution for this needs to be implemented to ensure the basis functions are solutions to localized functions only.

The mentioned settings in section 5.1.1 have a negative influence on the simulator when it comes to capturing pressure gradient. Considering Darcy's law $\left(q=-\frac{k}{\mu} \nabla p\right)$ capturing the pressure gradient specifically is important. For future research this is an important topic to capture pressure gradients accurately while staying away from peaks and troughs in the simulation. An important step in this could be the introduction of corner point gridding to place vertices more flexibly.

As a closing remark for this research the following statements can be made:

- Geologically relevant fracture maps are introduced.

- Computational costs for multiscale methods have been decreased.

- Field scale simulation can be performed using the introduced multiscale methods.

- Geological uncertainty is the largest source of error as only fracture map analogues can be used.

With this in mind, engineers can use multiscale methods as described in this research to see how flow behaves in the subsurface. However, one should always keep in mind that simulating a real subsurface problem with the mentioned methodology is still a great challenge as the subsurface is very hard to characterize and model according to reality. 


\section{Bibliography}

[1] Pierre-Olivier Bruna, Julien Straubhaar, Rahul Prabhakaran, Giovanni Bertotti, Kevin Bisdom, Gregoire Mariethoz, and Marco Meda. A new methodology to train fracture network simulation using multiple point statistic, 102018.

[2] M. Lough, S. Lee, and J. Kamath. A new method to calculate effective permeability of gridblocks used in the simulation of naturally fractured reservoirs. SPE Reservoir Engineering, 12:219-224, 081997.

[3] Kevin Bisdom. Burial-related fracturing in sub-horizontal and folded reservoirs: Geometry, geomechanics and impact on permeability. PhD thesis, 112016.

[4] B. Berkowitz. Characterizing flow and transport in fractured geological media: A review. Advances in Water Resources, 25:861-884, 2002.

[5] S. Geiger-Boschung, S. K. Matthai, J. Niessner, and R. Helmig. Black-oil simulations for three-component, three-phase flow in fractured porous media. SPE J., 14:338-354, 2009.

[6] P. Dietrich, R. Helmig, M. Sauter, H. Hotzl, J. Kongeter, and G. Teutsch. Flow and transport in fractured porous media. Springer, 2005.

[7] Pierre-Olivier Bruna, Julien Straubhaar, Rahul Prabhakaran, Giovanni Bertotti, Kevin Bisdom, Gregoire Mariethoz, and Marco Meda. A new methodology to train fracture network simulation using multiple-point statistics. Solid Earth, 10:537-559, 042019.

[8] Pierre-Olivier Bruna, Nico Hardebol, Kevin Bisdom, Julien Straubhaar, Gregoire Mariethoz, and Giovanni Bertotti. 2d to 3d fracture network detection and forecasting in a carbonate reservoir analogue using multiple point statistics (mps). 102017.

[9] N. Hardebol and Giovanni Bertotti. Digifract: A software and data model implementation for flexible acquisition and processing of fracture data from outcrops. Computers Geosciences, 54:326-336, 042013.

[10] Juliette Lamarche, Arthur Lavenu, Bertrand Gauthier, Yves Guglielmi, and Océane Jayet. Relationships between fracture patterns, geodynamics and mechanical stratigraphy in carbonates (south-east basin, france). Tectonophysics, 581:231-245, 112012.

[11] National Research Council. Rock Fractures and Fluid Flow: Contemporary Understanding and Applications. The National Academies Press, Washington, DC, 1996.

[12] Mark D. Zoback. Reservoir Geomechanics. Cambridge University Press, 2007.

[13] Richard W. Allmendinger, Nestor Cardozo, and Donald M. Fisher. Structural Geology Algorithms: Vectors and Tensors. Cambridge University Press, 2011.

[14] Colleen A. Barton, Mark D. Zoback, and Daniel Moos. Identification of hydraulically conductive fractures from the analysis of localized stress perturbations and thermal anomalies. 1994. 
[15] S.B. Pluimers. Hierarchical fracture modeling approach. Msc thesis, TU Delft, 2015.

[16] A. Moinfar, A. Varavei, K. Sepehrnoori, and R. T. Johns. Development of an efficient embedded discrete fracture model for $3 \mathrm{~d}$ compositional reservoir simulation in fractured reservoirs. SPE J., 19:289-303, 2014.

[17] M. Tene, M.S. Al Kobaisi, and H. Hajibeygi. Algebraic multiscale solver for flow in heterogeneous fractured porous media. In SPE Reservoir Simulation Symposium, 2015.

[18] S.M. HosseiniMehr. Multilevel multiscale method for embedded discrete fracture modeling approach (f-mlm). Msc thesis, TU Delft, 2016.

[19] Hadi Hajibeygi, Dimitris Karvounis, and Patrick Jenny. A hierarchical fracture model for the iterative multiscale finite volume method. Journal of Computational Physics, 230(24):8729 $8743,2011$.

[20] M. Tene, S. Bosma, M.S. Al Kobaisi, and H. Hajibeygi. Projection-based embedded discrete fracture model (pedfm). Advances in Water Resources, 105:205 - 216, 2017.

[21] Matteo Cusini, Cor van Kruijsdijk, and Hadi Hajibeygi. Algebraic dynamic multilevel (adm) method for fully implicit simulations of multiphase flow in porous media. Journal of Computational Physics, 314:60 - 79, 2016.

[22] Mousa Hosseinimehr, Matteo Cusini, C. Vuik, and Hadi Hajibeygi. Algebraic dynamic multilevel method for embedded discrete fracture model (f-adm). Journal of Computational Physics, 373:324-345, 112018.

[23] K. Bisdom, G. Bertotti, and F.H. Bezerra. Inter-well scale natural fracture geometry and permeability variations in low-deformation carbonate rocks. Journal of Structural Geology, 97:23 - 36, 2017.

[24] E. Bonnet, O. Bour, N. E. Odling, P. Davy, I. Main, P. Cowie, and B. Berkowitz. Scaling of fracture systems in geological media. Reviews of Geophysics, 39(3):347-383, 2001.

[25] John Hooker, Stephen Laubach, and Randall Marrett. A universal power-law scaling exponent for fracture apertures in sandstones. Geological Society of America Bulletin, 126:1340-1362, 092014.

[26] Brian Lawn. Fracture of brittle solids; 2nd ed. Cambridge Solide State Science Series. Cambridge University Press, Cambridge, 1993.

[27] P Pollard, D.D; Segall. Theoretical displacements and stresses near fractures in rock : With applications to faults, joints, veins, dikes, and solution surfaces, 1987.

[28] Jan Vermilye and C. Scholz. Relation between vein length and aperture: J m. vermilye c. h. scholz. Journal of Structural Geology, 17:423-434, 031995.

[29] Kevin Bisdom, Giovanni Bertotti, and Hamidreza M. Nick. The impact of different aperture distribution models and critical stress criteria on equivalent permeability in fractured rocks. Journal of Geophysical Research: Solid Earth, 121(5):4045-4063, 2016.

[30] Stephen F. Rogers. Critical stress-related permeability in fractured rocks. Geological Society, London, Special Publications, 209(1):7-16, 2003.

[31] R. Ulusay. The ISRM Suggested Methods for Rock Characterization, Testing and Monitoring: 2007-2014. 012015. 
[32] N. W. J. c. jaeger n. g. w. cook 1979. fundamentals of rock mechanics. 3rd edition. xix +593 pp., numerous figs. london: Chapman and hall. isbn 041222010 5. price £9.95. Geological Magazine, 117:401, 072009.

[33] J. Byerlee. Friction of Rocks, pages 615-626. Birkhäuser Basel, Basel, 1978.

[34] D W Peaceman. Interpretation of wellblock pressures in numerical reservoir simulation: Part 3; off-center and multiple wells within a wellblock. SPE (Society of Petroleum Engineers) Reservoir Engineering; (USA), 5(02).

[35] Yixuan Wang, Hadi Hajibeygi, and Hamdi A. Tchelepi. Algebraic multiscale solver for flow in heterogeneous porous media. Journal of Computational Physics, 259:284 - 303, 2014.

[36] Matei Țene, Mohammed Kobaisi, and Hadi Hajibeygi. Algebraic multiscale method for flow in heterogeneous porous media with embedded discrete fractures (f-ams). Journal of Computational Physics, 321, 062016.

[37] Bernd Flemisch, Inga Berre, Wietse Boon, Alessio Fumagalli, Nicolas Schwenck, Anna Scotti, Ivar Stefansson, and Alexandru Tatomir. Benchmarks for single-phase flow in fractured porous media. Advances in Water Resources, 111:239 - 258, 2018. 


\section{Appendix A}

\section{Mohr-Coulomb Plot}

In this section the Mohr-Coulomb plot is shown for the fracture set that has been used as a fieldscale test case. It is first mentioned in chapter 2 and afterwards in chapter 6 .

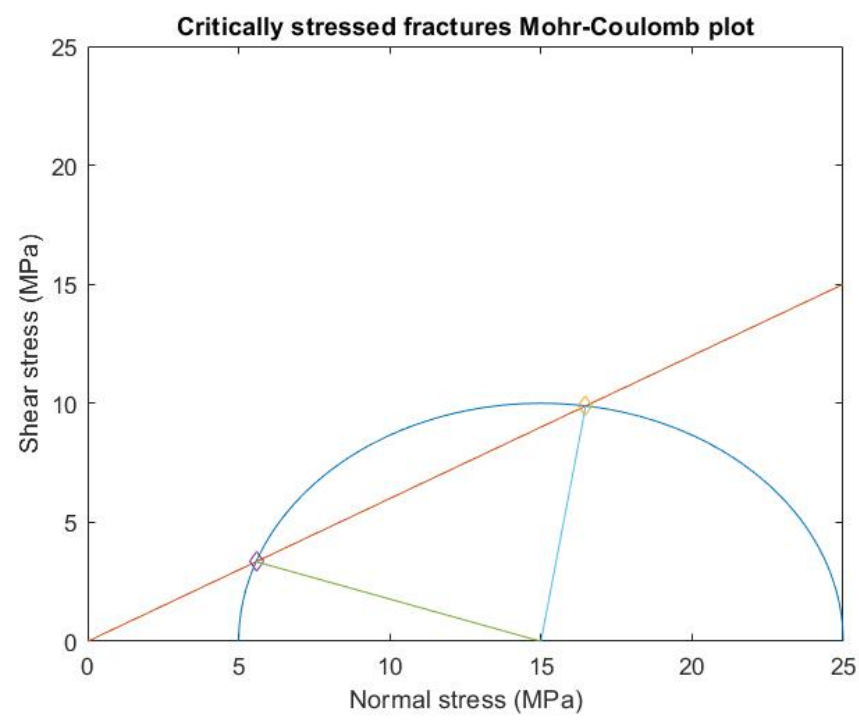

Figure A.1: The Mohr-Coulomb plot used for the classification of the fracture set mentioned in chapter 2 and 6 . 


\section{Appendix B}

\section{Principle Stress Settings}

In the table below, table B.1, the settings for the subsurface stress is shown. This has been used for the test case with the cross shape (i.e. test case 1) in section 5.2.

Table B.1: Table showing the principle stresses used to generate the fracture maps used in chapter 5

\begin{tabular}{|l|l|l|l|}
\hline Fracture permeability & $\sigma_{1}(\mathrm{MPa})$ & $\sigma_{3}(\mathrm{MPa})$ & Direction $\sigma_{1}$ \\
\hline Fully Impermeable Cross & 10 & 1 & 0 \\
\hline Full Permeable Cross & 3 & 1 & 60 \\
\hline $\begin{array}{l}\text { Horizontal Fracture Permeable } \\
\text { Vertical Fracture Impermeable }\end{array}$ & 10 & 1 & 150 \\
\hline $\begin{array}{l}\text { Vertical Fracture Permeable } \\
\text { Horizontal Fracture Impermeable }\end{array}$ & 10 & 1 & 60 \\
\hline
\end{tabular}




\section{Appendix C}

\section{Pressure Plot ADM Test Case}

Here the pressure solutions for the synthetic ADM test case is presented. The first plots belong to section 6.2 , to be followed by the plots belonging to section 6.3 .

\section{C.1. Flexible Coarsening Ratios for Fractures}

In the figure below, figure C.1, the pressure solutions are presented for a finescale multiphase simulation and a multilevel multiphase simulation in which all fractures are given a coarsening ratio of 3,9 and 15 respectively.

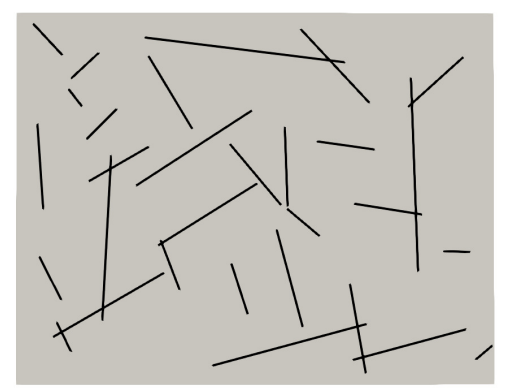

(a)

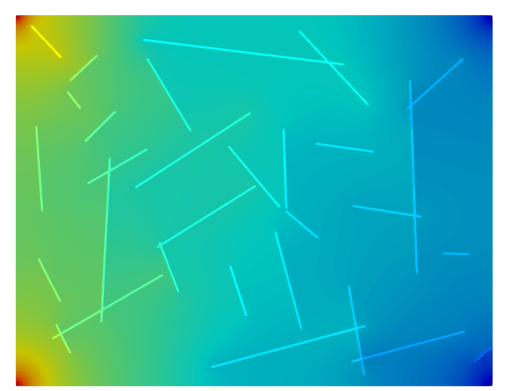

(b)

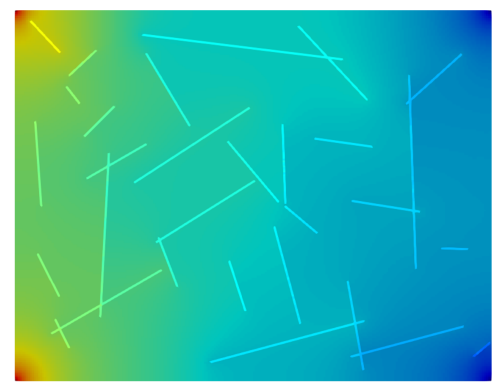

(c)

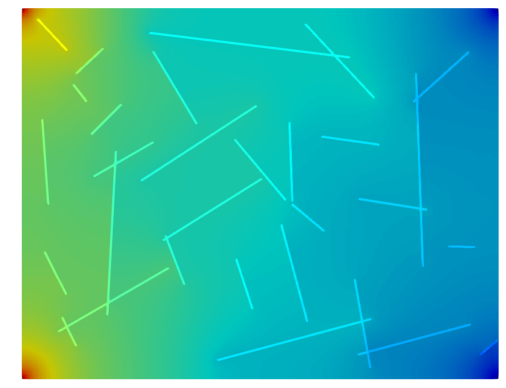

(d)

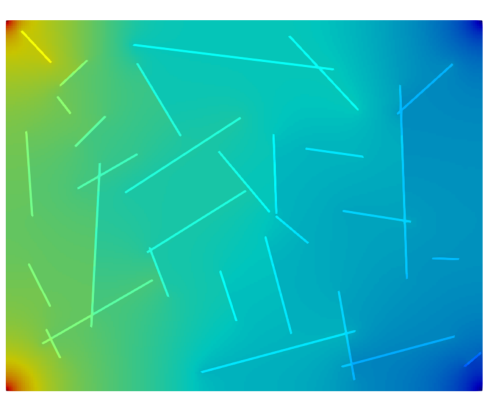

(e)

Figure C.1: (a) Fracture map (b) Finescale pressure solution (c) Coarsening ratio 3 for fractures (d) Coarsening ratio 9 for fractures (e) Coarsening ratio 15 for fractures. The figures b-e are a visualization of the reservoir after 150 days of injection and production. 


\section{C.2. Coupling of Fractures}

In the figure below, figure C.3, the pressure solutions are presented for a finescale multiphase simulation and a multilevel multiphase simulation in which either no, all or only permeable fracture gridcells are coupled to matrix gridcells with respect to the grid refinement scheme.

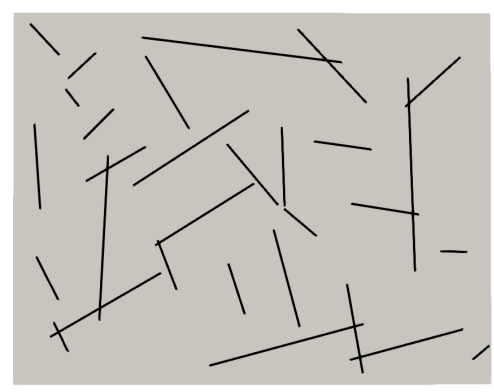

(a)

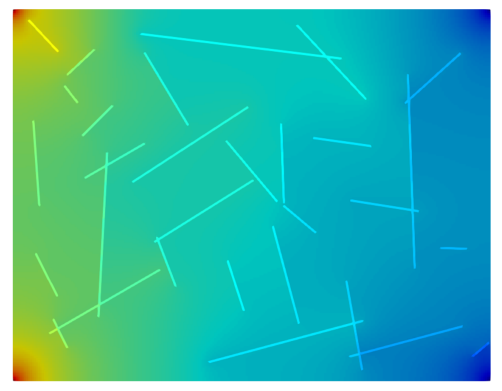

(b)

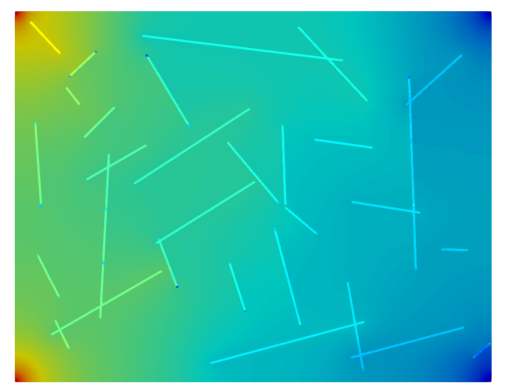

(c)

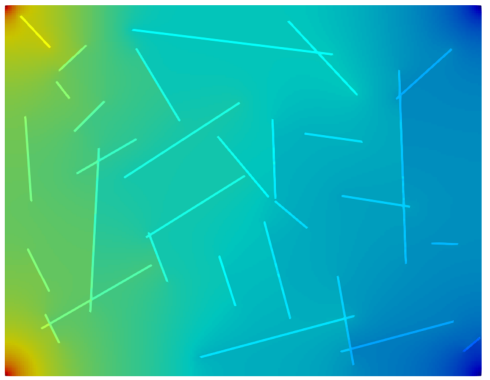

(d)

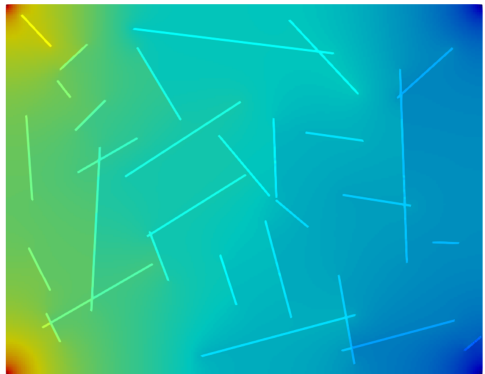

(e)

Figure C.2: (a) Fracture map (b) Finescale pressure solution (c) Decoupled (d) Fully coupled (e) Highly permeable fractures coupled. The figures b-e are a visualization of the reservoir after 150 days of injection and production. 


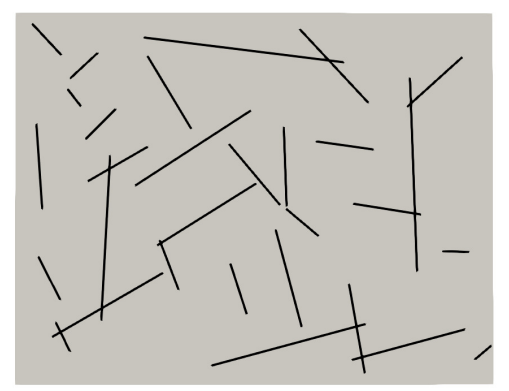

(a)

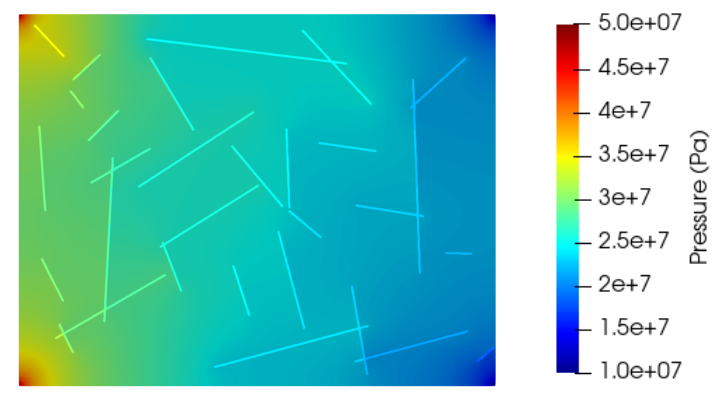

(b)

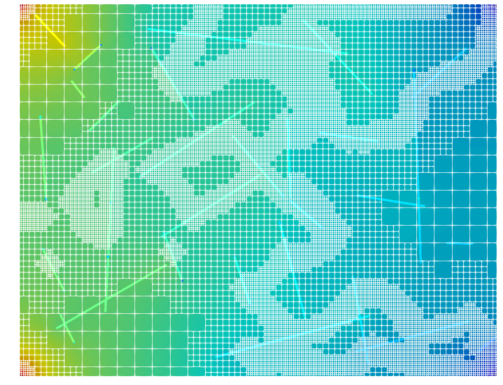

(c)

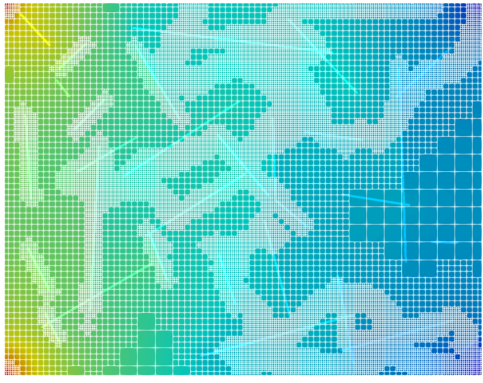

(d)

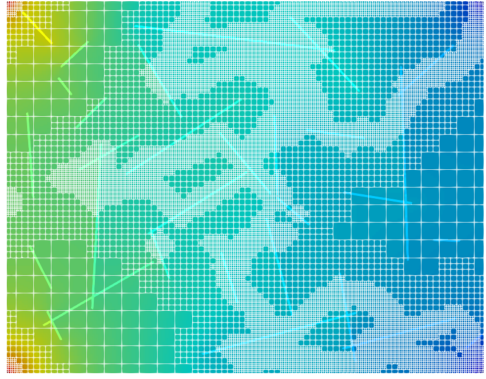

(e)

Figure C.3: (a) Fracture map (b) Finescale pressure solution (c) Decoupled (d) Fully coupled (e) Highly permeable fractures coupled. The figures b-e are a visualization of the reservoir after 150 days of injection and production. The gridding of the reservoir is plotted over the pressure solution to provide insight in how the front is captured by the grid refinement scheme. 